ACCEPTED MANUSCRIPT

\title{
Progress in 3D electrode microstructure modelling for fuel cells and batteries: Transport and electrochemical performance
}

To cite this article before publication: Duo Zhang et al 2019 Prog. Energy in press https://doi.org/10.1088/2516-1083/ab38c7

\section{Manuscript version: Accepted Manuscript}

Accepted Manuscript is "the version of the article accepted for publication including all changes made as a result of the peer review process, and which may also include the addition to the article by IOP Publishing of a header, an article ID, a cover sheet and/or an 'Accepted

Manuscript' watermark, but excluding any other editing, typesetting or other changes made by IOP Publishing and/or its licensors"

This Accepted Manuscript is @ 2019 IOP Publishing Ltd.

During the embargo period (the 12 month period from the publication of the Version of Record of this article), the Accepted Manuscript is fully protected by copyright and cannot be reused or reposted elsewhere.

As the Version of Record of this article is going to be / has been published on a subscription basis, this Accepted Manuscript is available for reuse under a CC BY-NC-ND 3.0 licence after the 12 month embargo period.

After the embargo period, everyone is permitted to use copy and redistribute this article for non-commercial purposes only, provided that they adhere to all the terms of the licence https://creativecommons.org/licences/by-nc-nd/3.0

Although reasonable endeavours have been taken to obtain all necessary permissions from third parties to include their copyrighted content within this article, their full citation and copyright line may not be present in this Accepted Manuscript version. Before using any content from this article, please refer to the Version of Record on IOPscience once published for full citation and copyright details, as permissions will likely be required. All third party content is fully copyright protected, unless specifically stated otherwise in the figure caption in the Version of Record.

View the article online for updates and enhancements. 


\title{
Progress in 3D electrode microstructure modelling for fuel cells and batteries: Transport and electrochemical performance
}

\author{
Duo Zhang, ${ }^{\mathrm{a}}$ Antonio Bertei, ${ }^{\mathrm{b}}$ Farid Tariq,,${ }^{\mathrm{c}, \mathrm{d}}$ Nigel Brandon, ${ }^{\mathrm{c}}$ Qiong Cai ${ }^{\mathrm{a}}$

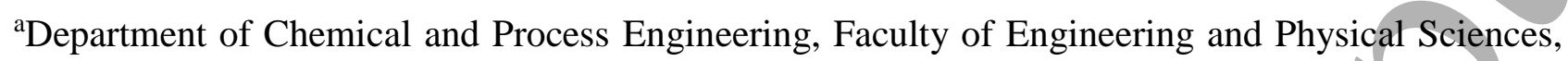 \\ University of Surrey, Guildford GU2 7XH, UK \\ ${ }^{\mathrm{b}}$ Department of Civil and Industrial Engineering, University of Pisa, Largo Lucio Lazzarino 2, Pisa \\ 56122 , Italy \\ ${ }^{\mathrm{c}}$ Department of Earth Science and Engineering, South Kensington Campus, Imperial College London, \\ London SW7 2AZ, UK \\ ${ }^{\mathrm{d} A d d i o n i c s, ~ W h i t e ~ C i t y, ~ L o n d o n, ~ W 12 ~ 0 B Z, ~ U K ~}$ \\ *Corresponding author: Email: q.cai@surrey.ac.uk; Tel: +44 (0) 1483686561
}

\begin{abstract}
Electrode microstructure plays an important role in the performance of electrochemical energy devices including fuel cells and batteries. Building a clear understanding of how the performance is affected by the electrode microstructure is necessary to design the optimal electrode microstructure, to achieve better device performance. $3 \mathrm{D}$ microstructure modelling enables us to perform simulations directly on a 3D electrode microstructure and thus link structure with performance. This paper provides an extensive review on the current state of the art in 3D microstructure modelling of transport and electrochemical performance for four promising electrochemical energy technologies: solid oxide fuel cells (SOFCs), proton exchange membrane fuel cells (PEMFCs), redox flow batteries (RFBs) and lithium ion batteries (LIBs). Each technology has different electrode microstructures and processes, and thus presents different challenges. The most commonly used modelling methods including the finite element method (FEM) and the finite volume method (FVM) are reviewed, together with the developing lattice Boltzmann method (LBM), with the advantages and disadvantages of each method revealed. Whilst FEM and FVM have been extensively applied in simulating SOFC and LIB electrodes where the methods are capable of dealing with single phase (gas or liquid) transport, they face challenges in simulating the multiphase phenomenon present in PEMFC and some RFB electrodes. LBM is, on the other hand, well suited in simulating gas-liquid two phase flow and applications in PEMFCs and RFBs, as well as single-phase phenomenon in SOFCs and LIBs. The review also points to current challenges in 3D microstructure modelling, including the
\end{abstract}


simulations of nanoscale gas transport and phase transition, moving interfaces associated with structural changes, accurate reactions kinetics, experimental validation, and how to make 3D microstructure modelling truly impactful through the design of better electrochemical devices.

Keywords: 3D microstructure modelling, Transport, Electrochemical performance, Solid oxide fuel cells, Proton exchange membrane fuel cells, Redox flow batteries, Lithium ion batteries

\section{Introduction}

Electrochemical energy devices including fuel cells and batteries are clean, sustainable and highly efficient power sources which are becoming increasingly important in the low carbon energy transition. Both fuel cells and batteries work via electrochemical reactions, meaning that they do not produce noise and harmful gases / particulates as heat engines do. Furthermore, energy generation via electrochemistry is usually more thermodynamically efficient than combustion alternatives. Fuel cells generate electricity by consuming hydrogen or hydrocarbon fuels and oxygen (in the air), whilst emitting only heat and water (and $\mathrm{CO}_{2}$ in the case of using hydrocarbon fuels). Batteries store electricity from the grid or from renewable sources such as wind and solar, and can supply the stored electricity on demand. There are various types of fuel cells and batteries, which vary in terms of the materials, the working temperature and the working principles, which then determine the most suitable applications. This review mainly focuses on solid oxide fuel cells (SOFCs), proton exchange membrane fuel cells (PEMFCs), redox flow batteries (RFBs), and lithium ion batteries (LIBs). SOFCs, working at relatively high temperatures $\left(500-1000{ }^{\circ} \mathrm{C}\right)$, are considered as promising power sources for distributed electricity generation and have been used for combined heat and power (CHP) applications [1,2]. PEMFCs, operating at relatively low temperatures $\left(80-100{ }^{\circ} \mathrm{C}\right)$, find most of their applications in small backup power sources and green hydrogen fuel cell electric vehicles such as buses, cars, boats, and trains, due to their ability to quickly respond to the power demand $[3,4]$. RFBs have tuneable power and energy density and are more suitable in grid energy storage applications [5-7]. LIBs have been widely used in portable electric devices and are considered as the ultimate choice for electric vehicles, due to their relatively high energy / power density combination, which are suitable for automotive applications [8,9].

Although there has been considerable development and deployment, the wide-spread applications of these electrochemical energy technologies remain hindered by their relatively high cost, durability, and the need to further improve energy / power density [1-9]. Continued research and development is needed to improve the available devices and to innovate new concepts, and this is underpinned by the development of materials which are the key to improving performance. A lot of research has 
therefore been dedicated to developing new materials chemistry, particularly new electrode materials which hold the key to energy and power density [2,9-12]. More recently, the importance of the physical structure (alongside the chemistry) of the electrode materials has also been recognized [1320]. Interestingly, different electrochemical energy devices share common features for their electrodes: regardless of the difference in materials chemistry, they all have a complex porous structure to facilitate the transport of electrolyte species, electrons, and ions, with electrochemical reactions happening across the volume of the electrode. The interplay of the physical properties of the porous structure (such as pore size, pore size distribution, porosity, tortuosity, particle size, and shape) affect the performance of the electrode and thus the device. To understand the effect of these physical properties on the transport and electrochemical performance of the electrode, considerable efforts have been made using both experimental and modelling approaches [13-20]. The experimental approaches are often trial-and-error; the processes of manufacturing electrodes with different structure properties, and measuring the electrochemical performance, prove to be time consuming. Often the electrodes are insufficiently characterised due to the lack of proper instruments, whilst the measurement of the distribution of electrochemical performance within a 3D electrode is challenging. On the other hand, modelling approaches have been úsed to provide an elegant way to relate the electrode properties to the performance [21], providing insights where experiments are difficult. Different modelling approaches exist, including continuum models [22-28], microstructure resolved models [29-38], and molecular/atomic models [39-42]. Molecular/atomic models are not included in this review, as they do not provide porous structure information such as porosity and particle size, due to the limited scale. Continuum models are only briefly mentioned here, due to their shortcomings as discussed below. This review focuses on the microstructure resolved modelling activities, as they are able to relate specific porous structures to performance.

Continuum models were firstly employed, in which the porous structure is treated as an effective medium, and bulk structure properties such as porosity, pore size, and tortuosity are input values to the models [22-28]. These continuum models are useful as they provide a quick way to manipulate the bulk structure properties and feedback to the performance. Continuum models normally require a large number of input parameters: some physical and microstructural parameters can be independently measured, while others cannot be determined directly, and thus the development of the models requires parameter fitting to find the right set of parameters for a particular system. Generally speaking, using a model to fit an unknown parameter may mask the shortcomings of the model itself, thus resulting in an inaccurate estimation of the parameter and in ambiguous model predictions, especially in multi-physics simulations relying on parameters obtained by different sources. Furthermore, since the porous structure is treated as an effective medium, the structure properties are 
only averaged values which do not reflect the heterogeneous nature of the porous structure. The simulations cannot give insights to how a localised artefact within the electrode affects the transport and reactions.

In the past decade, three-dimensional (3D) electrode microstructure resolved modelling, enabled by improved computational power, has become more and more popular [29-38]. Such models use a 3D electrode microstructure (either artificially generated in computers [29,34-36], or reconstructed from real electrodes using imaging techniques [33,37,38,43-47]) as the input of the model. The transport and electrochemical reactions are simulated directly on the 3D microstructure. This enables a direct link of the electrode structure to the electrode performance, with the capability to fully examine the heterogeneous structure, and its effect on the transport and reactions at local positions within the electrode.

This paper provides a review of different simulation methods which have been developed for modelling transport and electrochemical performance within the $3 \mathrm{D} /$ electrode microstructure. The review will cover different electrochemical devices including SOFCs, PEMFCs, RFBs, and LIBs. These technologies have different technical challenges and the review will shed light on how we can learn from the development in one technology and apply critical insights to another. The paper is organized as follows. In Section 2, these technologies are briefly introduced to explain their working principles and characteristics of electrode structures. In Section 3, the simulations of the multiphysical and chemical processes within the 3D electrode microstructures are reviewed, with different simulation techniques explained. In Section 4, the scientific findings achieved using the 3D electrode microstructure modelling for each of the technologies are reviewed and discussed. Section 5 draws conclusions and provide prospects for future research in this area.

\section{Fuel cell and battery technologies and their electrodes}

In this section, we wish to provide a brief description of the working mechanisms of the four selected technologies (SOFCs, PEMFCs, RFBs, and LIBs), as well as the electrode structures required for such devices.

\subsection{Solid oxide fuel cells (SOFCs)}

Different fuel cells are classified based on the nature of the electrolyte $[48,49]$, which enables transfer of ions between the electrodes while being electrically insulating. SOFCs are named after their solid, ceramic, oxide electrolyte which enables the conduction of oxygen ions at relatively elevated temperaturés (typically, greater than $500{ }^{\circ} \mathrm{C}$ ) [2,50]. 
Figure 1 (a) shows the schematic of the operation principle of an SOFC cell. Taking the example of hydrogen as a fuel, the global fuel oxidation reaction is decomposed into two half-reactions taking place at the anode and cathode, respectively:

$$
\begin{aligned}
& H_{2(g)}+O_{(e y)}^{2-} \rightarrow H_{2} O_{(g)}+2 e_{(a n)}^{-} \\
& \frac{1}{2} O_{2(g)}+2 e_{(c a)}^{-} \rightarrow O_{(e y)}^{2-}
\end{aligned}
$$

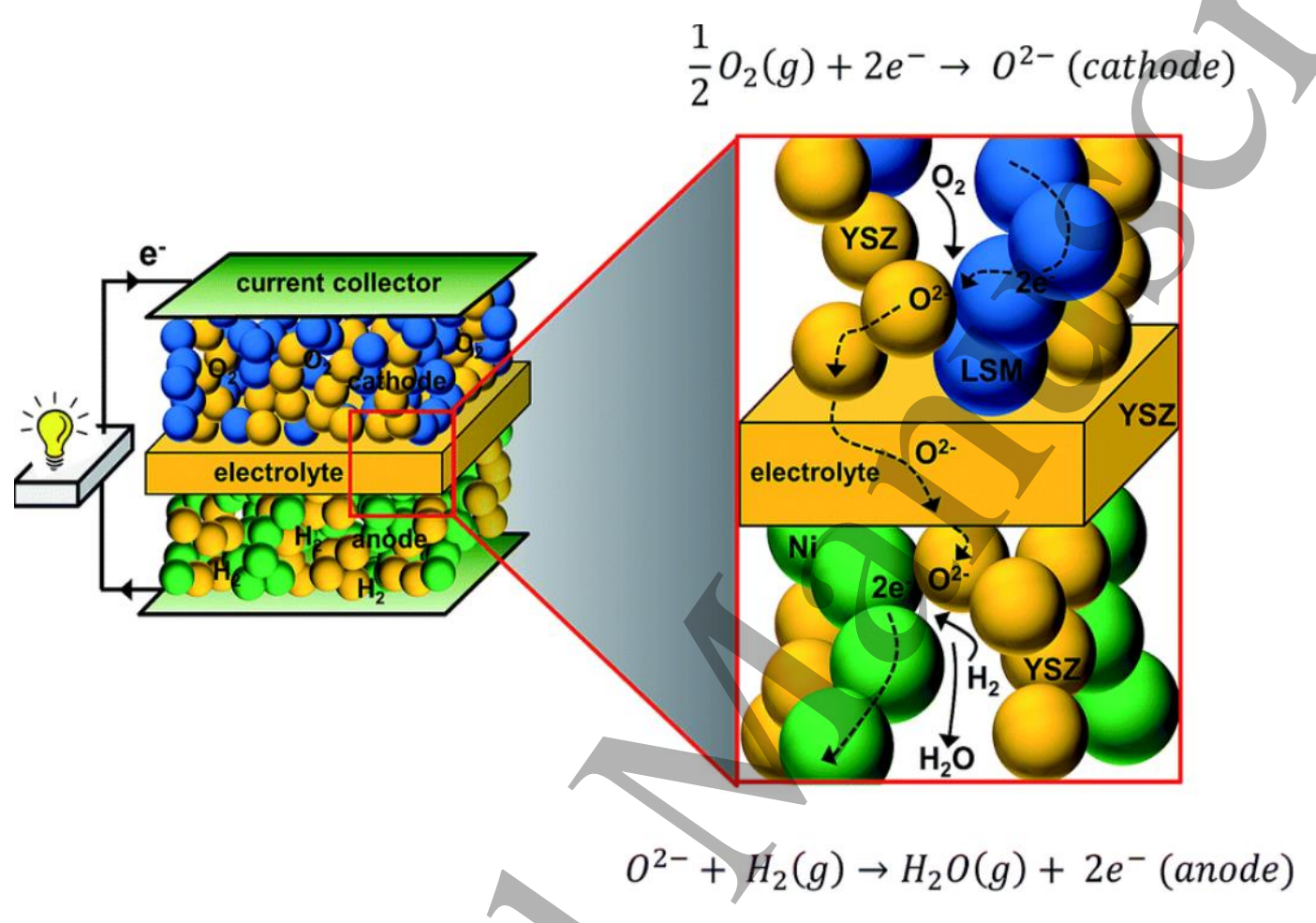

Figure 1: Schematic illustration of the operating principle of an SOFC cell, porous composite SOFC electrodes (reproduced from [51]).

According to these electrochemical reactions, chemical species $\left(\mathrm{H}_{2}, \mathrm{O}_{2}\right.$, and $\left.\mathrm{H}_{2} \mathrm{O}\right)$ are in the gas phase $(g)$, oxygen ions are carried by the electrolyte phase (ey), while electrons are transported by electronic conductors ( $a n$ and $c a$ ). Composite materials of metals and ceramics (so called cermet) are often used to generate both oxide ion and electronic conductivity, in which case, charge-transfer reactions can only occur where the chemical and charged species (gases, ions, and electrons) meet at the so-called triple phase boundaries (TPBs). When a mixed ionic-electronic conductor material (MIEC, generally a ceramic not a composite) is used, which is capable of transferring both $\mathrm{O}^{2-}$ and $\mathrm{e}^{-}$, the charge-transfer reactions happen at the dual phase boundaries (DPBs) which are interfaces. Thus, quantifying both TPB lines and interfaces in real 3D microstructures are important [52-54]. 
This basic description provides a simple picture of the main requirements of an SOFC electrode. The electrode must provide a sufficiently large number of reaction sites (TPBs or DPBs) per unit volume to promote the charge transfer reaction, while ensuring connected and easy access for chemical, ionic and electronic species to the reaction sites. Notably, TPBs or DPBs which are not connected to the source/sink of chemical and charged species are inactive [55]. Porous composites electrodes, typically made by a random dispersion of ion-conducting and electron-conducting particles, are manufactured to meet these requirements. Figure 1 (b) shows a cartoon illustration of such a porous composite electrode. Typical materials include ceramic oxide electrolytes (e.g., yttria-stabilised zirconia (YSZ), gadolinium-doped ceria (GDC)), ceramic electrode materials (e.g., lanthanum strontium cobalt ferrite, LSCF), and metallic conductors (e.g., nickel) [10,56].

Due to the complexity of the system, it is quite challenging to characterise the electrode microstructure and optimise it to maximise the overall energy conversion/efficiency. Critical tasks comprise: i) the determination of the effective transport properties in terms of tortuosity, conductivity, and constrictivity of transport pathways [57-59]; ii) the quantification of the density of active sites and the fraction of connected ones $[13,14,60]$; iii) the mechanistic description of the transport and reaction phenomena of multiple species in different regimes within a complex microstructure $[15,22,61,62]$; iv) the prediction of how the interplay between microstructural properties and electrochemical phenomena vary with time and affect the structural and chemical degradation mechanisms [63-65]. In this regard, modelling represents a useful tool to shed light on all these critical challenges and assist in device development.

\subsection{Proton exchange membrane fuel cells (PEMFCs)}

A PEMFC utilizes a proton exchange membrane as the electrolyte and the operation temperature is around $80-100{ }^{\circ} \mathrm{C}$. Figure 2 (a) shows a schematic of a working PEMFC, in which hydrogen gas at the anode side is converted to protons. This oxidation half-cell reaction or hydrogen oxidation reaction is represented by:

$$
H_{2} \rightarrow 2 H^{+}+2 e^{-}
$$

The newly formed protons permeate through the polymer electrolyte membrane to the cathode side to combine with oxygen and generate water, while electrons travel along an external load circuit to the cathode side, creating the current output of the fuel cell. This reduction half-cell or oxygen reduction reaction is represented by:

$$
\frac{1}{2} \mathrm{O}_{2}+2 \mathrm{H}^{+}+2 e^{-} \rightarrow \mathrm{H}_{2} \mathrm{O}
$$




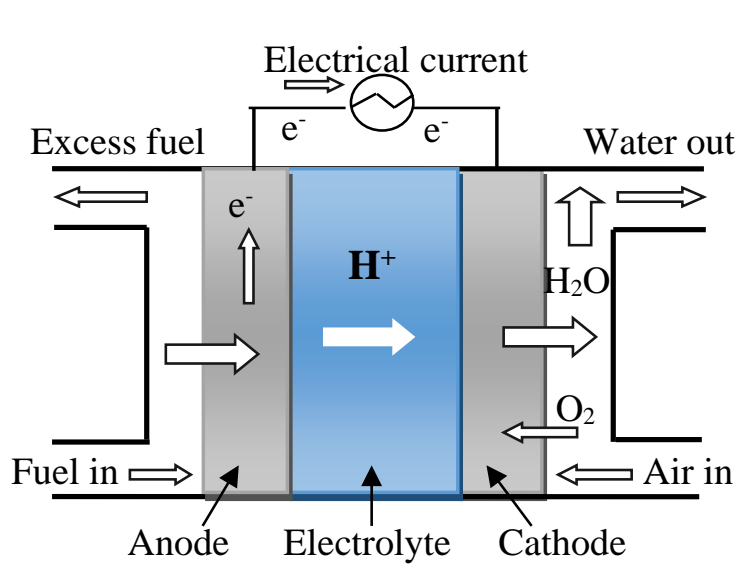

(a)

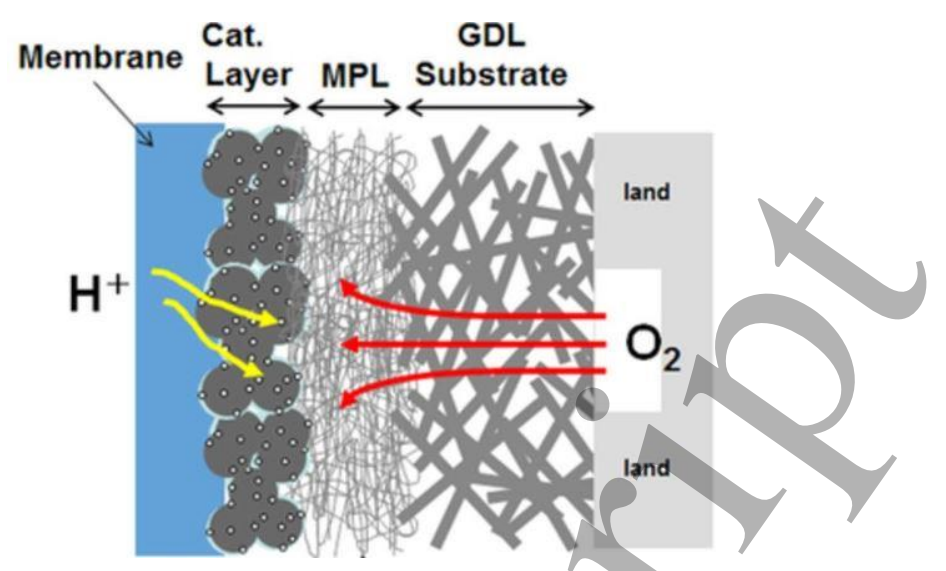

(b)

Figure 2: (a) Schematic of a typical hydrogen PEMFC and (b) cross-sectional view of the components of cathode and their electrochemical contributions [reproduced from 16].

Figure 2 (b) shows the schematic of a typical electrode structure in PEMFCs. Both anode and cathode have a multi-layer structure, namely a gas diffusion layer (GDL), a microporous layer (MPL) and a catalyst layer (CL), to fulfill different functions. The CL, with an/overall thickness of 5-20 $\mu \mathrm{m}$ [3], consists of platinum/carbon $(\mathrm{Pt} / \mathrm{C})$ catalysts, ionomers and pores to form mostly heterogeneous interconnected transport paths for electrons, ions, and reactants, respectively, to facilitate electrochemical reactions. Careful design is required to ensure sufficient reaction sites, i.e. good contacts between catalysts, ionomers, and pores. The porous GDL, commonly carbon paper or woven carbon fabrics [4], are placed on either side of the membrane in a PEMFC to ensure that reactants effectively diffuse to the catalyst layer, and assist electron conduction to and from the CL [66]. Under high current density conditions, oxygen transport loss in the cathode can account for a substantial portion of the performance loss [17]. The oxygen transport loss is triggered by the electrode flooding when the produced liquid water blocks pores in the GDL and covers the electrochemically active sites in the CL, thereby increasing surface overpotential. The CL and GDL, therefore, play a crucial role in PEMFC water management. The MPL, usually fabricated by intermixing a hydrophobic agent (e.g., polytetrafluoroethylene (PTFE)) with carbon black, is placed between the CL and the GDL [11], to enhance cell water management and improve the performance $[67,68]$. The pore-scale modelling is a significant tool that can assist in optimizing the porous structure of the CL, MPL, and the GDL, with the aim to maintain a delicate and functioning balance between reactant transport from the gas channels and water removal from the electrochemically active sites. 
RFBs, as an efficient energy storage technology, provide many advantages such as long cycle life, high reliability, independently tunable power and energy, low cost, and simplified manufacturing [57]. Figure 3 (a) shows a simplified schematic of a RFB. A typical RFB consists of an anolyte reservoir and a catholyte reservoir, from which the anolyte and catholyte are circulated by pumping through two carbon-based porous electrodes, where electrochemical reductions and oxidation of redox couples occur separately at anode and cathode, during charge and discharge respectively [12]. Both anolyte and catholyte can be generalized as "electrolyte". For the most commonly used vanadium $\mathrm{RFB}$, an anolyte contains $\mathrm{V}^{2+}$ and $\mathrm{V}^{3+}$ ions, whilst a catholyte contains $\mathrm{VO}_{2}{ }^{+}$and $\mathrm{VO}^{2+}$ ions dissolved in a sulfuric acid $\left(\mathrm{H}_{2} \mathrm{SO}_{4}\right)$ solution. During the discharge cycle, the following reactions take place at the surface of the carbon fibres [30].

Negative half cell: $\mathrm{V}^{2+} \rightarrow \mathrm{V}^{3+}+\mathrm{e}^{-}$
Positive half cell: $\mathrm{VO}_{2}{ }^{+}+2 \mathrm{H}^{+}+\mathrm{e}^{-} \rightarrow \mathrm{VO}^{2+}+\mathrm{H}_{2} \mathrm{O}$

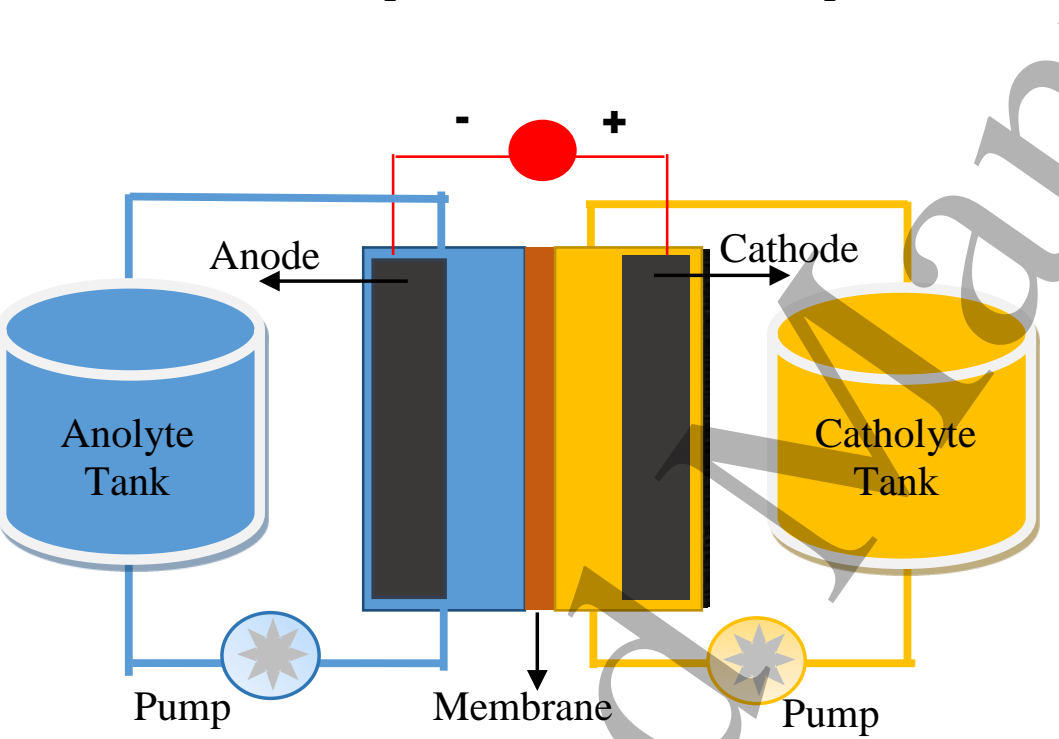

(a)

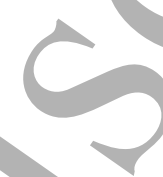

Figure 3: (a) Simplified schematic of a vanadium redox flow battery; (b) bubble evolution in a fibrous electrode [69].

The electrodes in RFBs are responsible for providing active sites for redox reactions and facilitating the distribution of chemical species. State-of-the art commercially available RFB electrodes consist of randomly distributed or aligned carbon fibres that form porous carbon mats with varying microstructural properties, and include non-woven structures (e.g., carbon papers and felts) and woven carbon cloths [18]. Such materials show complex microscopic porous structures, in which fibres contact, overlap or intersect with each other, leading to non-uniform pore size distributions. Therefore, the properties of the electrodes, in particular, their microstructure, play an important role in determining the mass transfer and pressure drop of electrolyte flow through electrodes. 
Understanding of the relationship between the electrode microstructure and RFB performance remains in its infancy when compared to SOFCs and PEMFCs due to several challenging reasons, including the difficulty in seeing the subtle differences that occur in the RFB microstructure through current imaging methods. For the first time known to the authors it has only been possible very recently to dynamically see the liquid flow in RFB electrodes [8]. The understanding of the performance is enhanced through continuously evolving experimental methods (as different systems are built) and complexity in modelling (e.g. multiple-phase flow inside the porous electrode [69] during the liquid electrolyte wetting process, and hydrogen gas bubbles produced from side reactions during battery operations). Mass transfer losses can lead to large concentration overpotentials due to the inability to deliver adequate reactants to the surface of the electrode. Moreover, as shown in Figure 3 (b), in some cases, air bubbles produced from side reactions can occupy the pore space used for electrolyte transport, preventing reactant species reaching active surface, and further increasing the overpotential loss. As the concentration overpotential of the cell and pressure drops increases, the overall efficiency is reduced. In addition, it is worth highlighting that bubble pockets can accelerate localized degradation of the carbon fibres. So, how to evaluate and design the microstructure of electrode to improve the overall efficiency of RFBs is quite challenging. Pore-scale modelling provides an efficient way for in-depth understanding of the multiple processes in porous electrodes.

\subsection{Lithium ion batteries (LIBs)}

LIBs represent the current state-of-the-art technology for secondary batteries that can provide a good combination of high power and energy densities with acceptable lifetime, making them suitable for a wide variety of energy applications. The energy density of a rechargeable LIB relies on the quantity of active material which is capable of storing $\mathrm{Li}$ in the negative electrode (i.e., the anode during discharge) and accommodating $\mathrm{Li}^{+}$in the positive electrode (i.e., the cathode during discharge), thus shuffling $\mathrm{Li}^{+}$between the electrodes according to the following simplified electrochemical reactions (see Figure 4 (a)) [9,19]:

$$
\begin{aligned}
& L i_{x} C_{y} \leftrightarrow x L i_{(e y)}^{+}+x e^{-}+y C \\
& x L i_{(e y)}^{+}+x e^{-}+L i_{1-x} M O_{z} \leftrightarrow L i M O_{z}
\end{aligned}
$$

where $\mathrm{C}$ represents the negative electrode material, typically graphite, and $\mathrm{LiMO}_{\mathrm{z}}$ is the positive

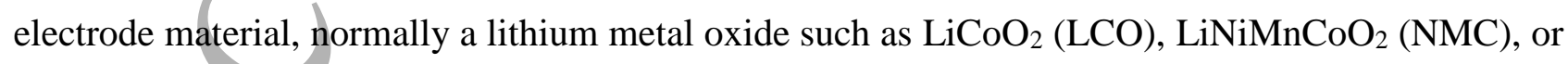
$\mathrm{LiFePO}_{4}(\mathrm{LFP})[9,70] . \mathrm{Li}^{+}\left(\right.$ey) is the cation of a lithium salt (e.g., $\left.\mathrm{LiPF}_{6}\right)$ dissolved in a mixed organic solvent (e.g., ethylene carbonate-dimethyl carbonate, EC-DMC) [9]. Power density is determined by the rate at which energy can be extracted or stored in the battery and is more fundamentally linked to diffusion and interfacial contacts in battery electrodes. Electrons are carried from/towards the current 
collector plates by the electrode material if it is sufficiently electronically conductive (as in the case of graphite) and/or by conductive additives mixed with the active material particles to enhance electron transport throughout the electrode thickness (Figure 4(b)).

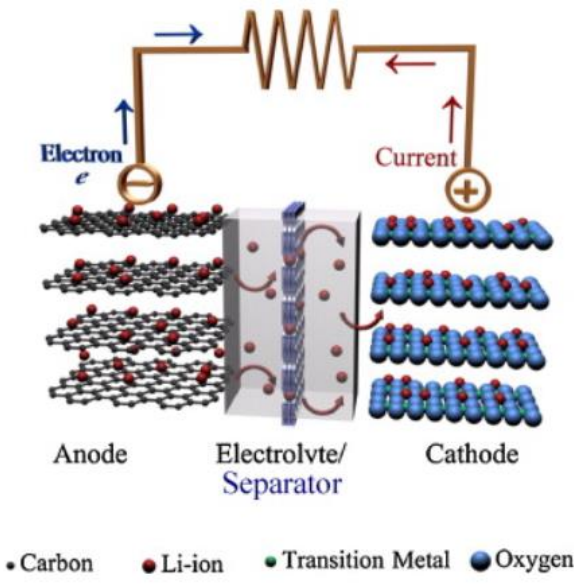

(a)

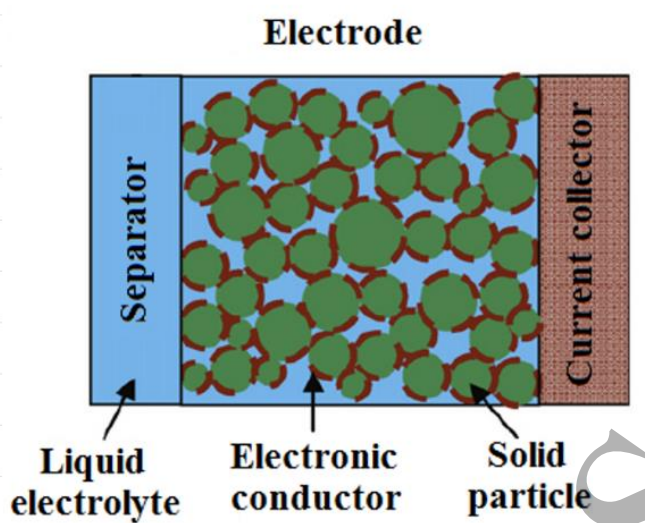

(b)

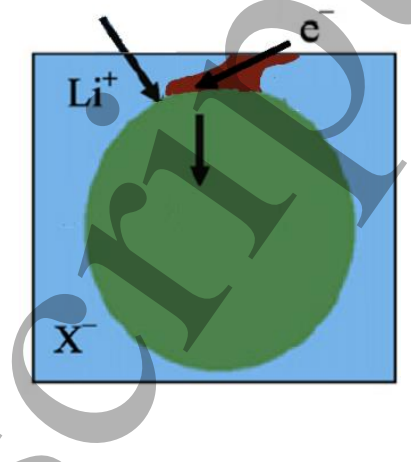

(c)

Figure 4: Schematic illustration of (a) a typical lithium-ion battery with graphite as anode and a transition metal oxide as a cathode[70], (b) a porous LIB electrode with conductive additives, c) electrochemical intercalation reaction at the particle surface along with diffusion of lithium within the particle [adapted from [24]]

The reactions in Eq. (2.4a) occur from left to right during discharge (i.e., when the battery provides electrical energy) and from right to left during charge (i.e., when the battery stores electric energy to recover the initial charged state). In a fully charged LIB, lithium is stored as a Li complex within the particles of the negative electrode. During discharge, Li diffuses out of the particles and undergoes the de-intercalation reaction at the anode side/(Eq. (2.4a)), forming $\mathrm{Li}^{+} . \mathrm{Li}^{+}$ions diffuse and migrate within the liquid electrolyte phase throughout the anode and the separator towards the cathode, wherein the cathodic intercalation reaction (Eq. (2.4b)) takes place, enabling Li storage within the positive electrode particles. During charge, the same phenomena occur in the reverse direction, releasing electrons via an external circuit which can be used for useful work.

Regarding the LIB electrochemical performance, the electrodes must fulfil two functions: 1) storing the maximum amount of lithium per unit volume - this is related to the energy and capacity density of the system and;2) ensuring fast intercalation/de-intercalation processes - this is related to the power density of the battery [71]. The electrode microstructure is the key to exploiting both these functionalities. In fact, the volume fraction of active material within the electrode and the distribution of particles determine the accessible capacity of the battery [72]. On the other hand, other microstructural properties affect the rate of charge/discharge, such as: i) the size of the particles 
determines the rate of lithium intra-particle diffusion and the surface area available for the chargetransfer reaction [73]; ii) the volume fraction and tortuosity of the porous phase affects the rate of $\mathrm{Li}^{+}$ migration [74,75]; and iii) the connectivity of conductive additives determines the fraction of particles electronically connected to the current collector [76-78]. In addition, since typically lithium intercalation causes particle swelling, the electrode microstructure may change over several cycles [79]. The accessible capacity and power of a given battery may be different from its theoretical capacity and power. A large portion of this difference can be due to the way in which the electrodes are structured and put together in layers to form the battery. All these phenomena and their interplay with the electrode microstructure must be properly characterised and modelled to guide the rational design of optimised electrodes $[20,80]$.

\subsection{Imaging techniques for obtaining the 3D microstructure of fuel cells and battery electrodes} There are multiple forms of 3D imaging techniques that can be deployed to probe, investigate and capture the real structure and geometry of different electrodes. The precise suitability of which method to deploy depends on the nature of the sample and size of features that are of interest. Broadly they can be considered as 'true' tomography and 'pseudo-tomography.' The true tomography relies on transmission of typically electromagnetic waves and their reconstruction from sinograms to reproduce the actual object. Techniques in this category include X-ray computed tomography and electron tomography. These techniques are non-destructive towards the specimen they investigate. The pseudo-tomography physically slices the sample in a serial fashion while imaging the revealed face to reconstruct the object, or sputters atoms from the sample to determine their position. Techniques in this category include: focused ion beam-scanning electron microscopy (FIB-SEM), dual beam focused ion beam (DB FIB), and Atom Probe Tomography (APT). These are destructive towards the specimen they investigate. The volume and length scales the above techniques typically operate are shown in Figure 5 [81,82]. Progression in each technique continues to push for higher resolution and better accuracy. The 3D structure from different tomographic techniques can then be integrated together (multi-scale tomography) and used in modelling spanning very large ranges in length scales. This unique approach of incorporating multi-scale tomography and modelling reveals the nature of the sample's behavior that would not otherwise be apparent [83].

For most applications of electrochemistry and electrodes, the most relevant methods are X-ray computed tomography (X-ray CT) and FIB-SEM $[37,38,44-47,84]$. This is due to the nature of samples and feature sizes of most interest. Subsequent uses of the different tomographic methods in this review will mainly refer to these subcategories and scales. The electrodes in fuel cells and batteries under research are normally tens to hundreds micrometers in the thickness dimension, and 
centimers in the other two dimensions. The particle size in these electrodes can span from a few hundred nanometers $(\mathrm{nm})$ to a few hundred micrometers $(\mu \mathrm{m})$. For obtaining microstructures of such electrodes, a voxel scale (or resolution) of $10 \mathrm{~nm}$ to $50 \mu \mathrm{m}$ is considered, depending on the actual particle size and the accuracy required. Since it is highly time-consuming and costly to obtain an entire electrode, imaging techniques are often focused on a small region of interest within the electrode, for acquiring a representative electrode microstructure. The typical size of such representative microstructure can vary from $100 \mu \mathrm{m}^{3}$ to $10 \mathrm{~mm}^{3}$, as shown in Figure 5.

\section{Tomography Methods}

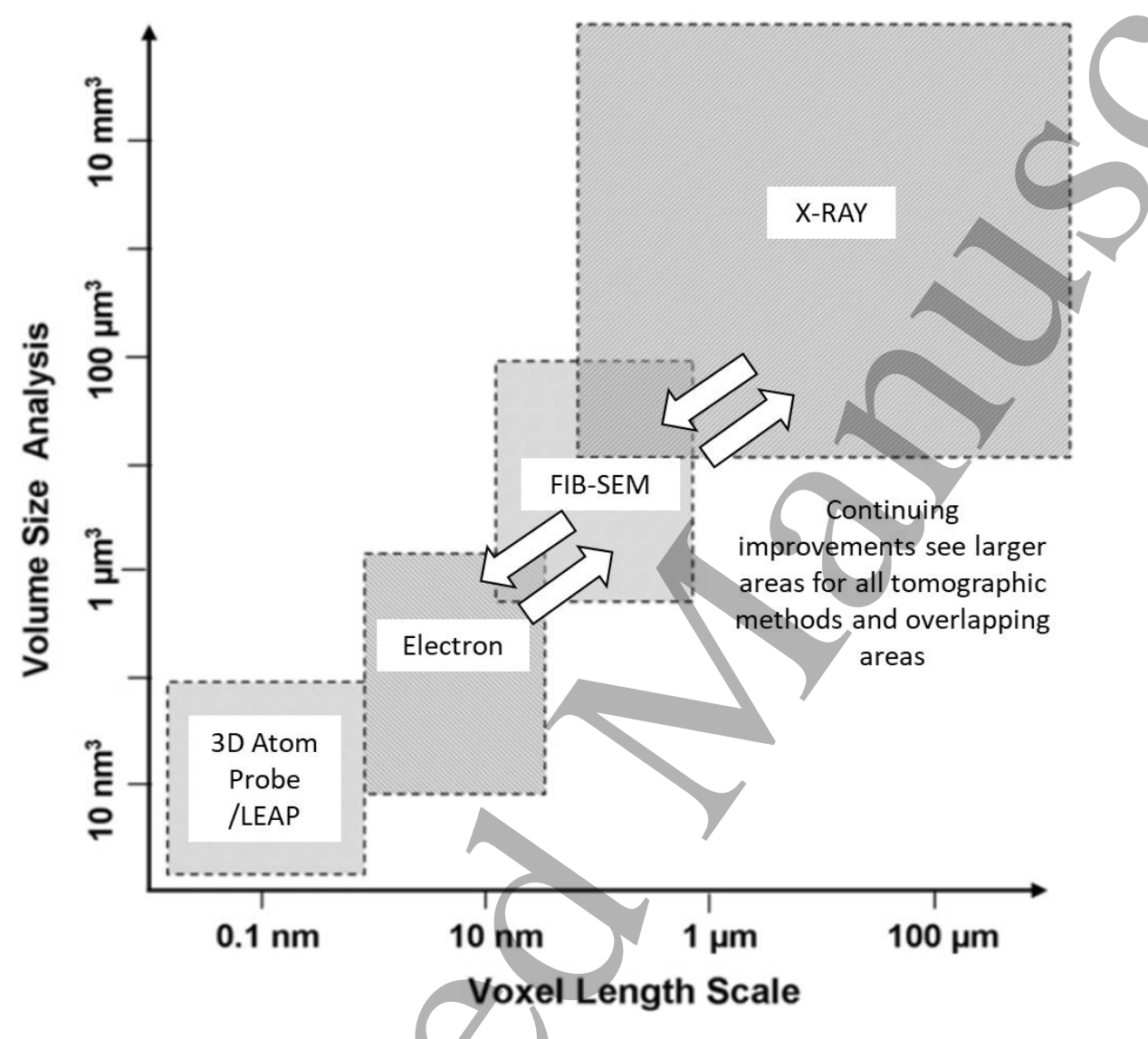

Figure 5: Different tomographic techniques with their typical resolution and volume size capabilities. The figure is adapted after [81].

\section{Transport modelling and electrochemical performance simulations}

In this section, we will review the fundamental mechanisms and governing equations for modelling transport and electrochemical performance within the 3D electrode microstructures of fuel cells and batteries. These 3D microstructures are complex, porous, tortuous, and composite, with materials and functions that are unique to each technology. Furthermore, the 3D microstructures adopted for the modelling normally have a domain size in the range of $100 \mu \mathrm{m}^{3}$ to $10 \mathrm{~mm}^{3}$, with a voxel size from 
$10 \mathrm{~nm}$ to $50 \mu \mathrm{m}$, as discussed in Section 2.5. This gives rise to: complex boundaries, additional phenomena comparing to macroscopic continuum modelling, and challenges in solving equations on such complex geometries. Adequate methods need to be developed, to deal with the multi-physics and reactions on complex geometries. To give a clear view of these issues, Section 3.1 reviews fundamental mechanisms and governing equations specific to each technology; whilst Section 3.2 reviews the development of the numerical methods for modelling transport and electrochemical performance on $3 \mathrm{D}$ electrode microstructures.

\subsection{Simulations of transport and reaction processes}

\subsubsection{SOFCs}

When a typical cermet material (which is a composite of metal and ceramic) is used for SOFC electrodes (e.g. Ni/YSZ as SOFC anode), simulations are required to consider the transport of electrons in $\mathrm{Ni}$ metal phase and transport of oxygen ions in YSZ ceramic phase, as well as the transport of gases in the pore phase. In the case of a ceramic material (such as GDC) acting as a mixed ionic-electronic conductor (MIEC), the transport of both electrons and ions within the ceramic materials need to be considered. Transport of electrons and ions are modelled considering the electronic and ionic potentials [85,86]:

$$
\begin{aligned}
& \nabla \cdot\left(\sigma_{e l} \nabla \phi_{e l}\right)=0 \\
& \nabla \cdot\left(\sigma_{i o n} \nabla \phi_{i o n}\right)=0
\end{aligned}
$$

where $\emptyset_{e l}$ and $\emptyset_{i o n}$ are electronic and ionic potentials, $\sigma_{e l}$ and $\sigma_{i o n}$ are conductivities in electron and ion conductors.

The transport of gases is modelled considering the concentration gradient [87]:

$$
\nabla\left(D \nabla C_{i}\right)=0
$$

where $C_{i}$ is the concentration of species $i$, and a singular diffusion coefficient $D$ suffices for gaseous reagents, $D$ is the bulk diffusion coefficient and is calculated by considering binary gas composition. For an SOFC anode where $\mathrm{H}_{2}$ reduction and $\mathrm{H}_{2} \mathrm{O}$ generation occur, a binary gas of $\mathrm{H}_{2} / \mathrm{H}_{2} \mathrm{O}$ is considered [29,87-89]:

$$
D=\frac{p_{\mathrm{H}_{2} \mathrm{O}}}{p} D_{\mathrm{H}_{2}}+\frac{p_{\mathrm{H}_{2}}}{p} D_{\mathrm{H}_{2} \mathrm{O}}
$$

where $p$ is the absolute pressure, and $p_{\mathrm{H}_{2} \mathrm{O}}, p_{\mathrm{H}_{2}}$ are partial pressures of $\mathrm{H}_{2} \mathrm{O}$ and $\mathrm{H}_{2}$. Notably, this approach is surely simplistic and does not consider transport mechanisms such as Knudsen diffusion, which is significant in case of nanopores. Knudsen diffusion has been explicitly considered by Lu $e t$ 
al. [15,90] via Direct Simulation Monte Carlo, although the diffusion simulation is not directly coupled to electrochemical reactions and other transport phenomena.

Electrochemical reactions take place at the triple phase boundaries (TPBs) in SOFC cermet electrodes. When a MIEC is used capable of transferring both $\mathrm{O}^{2-}$ and $\mathrm{e}^{-}$, the charge-transfer reactions happen at the dual phase boundaries (DPBs). The reaction current is given by the Butler-Volmer equation as follows [85-87].

$$
j=j_{0}\left[\exp \left(\alpha \frac{F}{R T} \eta\right)-\exp \left(-(1-\alpha) \frac{F}{R T} \eta\right)\right]
$$

where $j$ is the local current density at the triple phase boundary, $F$ is the Faraday's constant, $\alpha$ is the charge transfer coefficient, which is a measure of the symmetry of the energy barrier of the charge transfer reaction affecting the symmetry of the current-overpotential curve [91], and $\eta$ is the local activation overpotential which can be defined as:

$$
\eta=\phi_{e l}-\phi_{i o n}-E_{e q}
$$

where $E_{e q}$ is the local equilibrium potential difference, which depends on local gas partial pressures according to Nernst law [22,92]. It is noteworthy to mention that an alternative definition of activation overpotential, based on the local chemical potential difference between oxygen in the ceramic and gas phases as described by Mizusaki et al. [93,94], which eventually requires the electrochemical potentials of electrons and oxygen ions as field variables instead of $\phi_{e l}$ and $\phi_{i o n}$, has been used in both cathode [95,96] and anode [97] models.

The exchange current density $j_{0}$ is defined as (here referred to a hydrogen fuel electrode):

$$
j_{0}=k_{0} p_{\mathrm{H}_{2}}^{\beta} p_{\mathrm{H}_{2} \mathrm{O}}^{\gamma}
$$

where $p_{\mathrm{i}}$ is the local partial pressure of gas species $i$, while $\beta$ and $\gamma$ are dimensionless exponents and $k_{0}$ is the reaction rate which is dependent on the materials. The factors $\alpha, \beta, \gamma$, and $k_{0}$ are intrinsic properties of the materials and gas reactants, which are typically obtained by fitting of dedicated kinetic experimental measurements.

\subsubsection{PEMFCs}

The unique functions of the multilayer structure of the PEMFC electrodes require different modelling solutions for each layer. In the anode, simulation of the transport of $\mathrm{H}_{2}$ and water vapour through the GDL and MPL to the CL is necessary. In the cathode, the reactant water vapor condenses into liquid water in the CL and GDL. It is therefore important to simulate gas-liquid two phase flow in GDL and 
MPL, as these two layers facilitate the transport of $\mathrm{O}_{2}$ to the $\mathrm{CL}$, while carrying away water that has been generated at the CL. Transport of $\mathrm{H}_{2}, \mathrm{O}_{2}$ and water vapour in the GDL and MPL is described as the same as Eq. (3.1.1 c). The treatment of gas-liquid two phase flow will be discussed in Section 3.2, with specific numerical methods.

Apart from transporting gases and liquid, the GDL and MPL structures also transport electrons as they are electron conductors. The electronic potentials in electron conductors can be considered as Eq. (3.1.1 a).

The CL is a mixture of electron conductors, ion conductors and catalysts and has a complex porous structure. In addition to the transport of gases and electrons, transport of ions in ion conductors needs to be considered, as Eq. (3.1.1 b).

Within the CL, electrochemical reactions happen where electron conductors, ion conductors, catalysts and pore phase are connected. In the same manner as SOFCs, the Butler-Volmer equation (Eq. (3.1.1.e)) is used to get the local current density $j$ at active sites. Parallel reaction pathways or microkinetic schemes based on multiple reaction steps are seldom considered [98], such as the detailed oxygen reduction reaction mechanism comprising oxygen dissolution, diffusion within the ionomer film and reaction at the ionomer-catalyst interface applied by Sabharwal et al. [99]. This is owing to the complexity in including these factors and simpler models often providing sufficient correlation to experimental values for most studied requirements.

\subsubsection{RFBs}

In RFB electrodes, the reactive species are transported within the liquid electrolyte which is pumped through the electrode pore space, whilst electrochemical reactions happen at the interfaces of the liquid and the solid electrode materials. It is important to use the convection-diffusion equation to describe the transport of the reactive species in porous electrodes. The convection-diffusion equation can be written as [100]:

$$
\frac{\partial C_{i}}{\partial t}+\nabla \cdot\left(C_{i} \mathbf{u}\right)=\nabla \cdot\left(D_{i} \nabla C_{i}\right)+\nabla \cdot\left[\frac{z_{i} F C_{i} D_{i}}{R T} \nabla \varphi\right]
$$

where $C_{i}$ is the concentration of species $i$ in electrolyte, $D_{i}$ is the diffusivity of species $i$ in electrolyte and $\mathbf{u}$ is the velocity of the flow field $\left[\mathrm{m} \bullet \mathrm{s}^{-1}\right]$. The velocity of electrolyte plays a vital role in RFBs, which affect the supply rate of reactant and decide the performance of battery. The effect of migration 
on the species transport is described as $\frac{\nabla \cdot\left[\frac{z_{i} F C_{i} D_{i}}{R T} \nabla \phi\right]}{R T}, \phi$ is the electrical potential, $z_{i}$ is the charge of species and $F$ is the Faraday's constant.

Fibrous carbon materials are often used as RFB electrodes. Inside the solid carbon fibres, the governing equations for the potential field can be described as Eq. (3.1.1a)

In the electrolyte, the potential field can be obtained as:

$$
\nabla \cdot\left[\sigma_{i o n} \nabla \phi_{i o n}+F \sum_{j} D_{j} \nabla C_{j}\right]=0
$$

where the effective conductivity of electrolyte $\sigma_{\text {ion }}$ is defined as [30,101]:

$$
\sigma_{\text {ion }}=\frac{F^{2}}{R T} \sum_{j} z_{j}^{2} D_{j} C_{j}
$$

In porous electrodes, electrochemical reactions take place at the solid-liquid interfaces. ButlerVolmer kinetics is used to evaluate the local current density $j$ (referred per unit of surface area ), taking the negative of vanadium RFBs as an example [102]:

$$
j=k_{0} F\left(C_{V^{2+}}\right)^{\alpha}\left(C_{V^{3+}}\right)^{(1-\alpha)}\left[\frac{C_{V^{2+}}^{S}}{C_{V^{2+}}} \exp \left(\frac{(1-\alpha) F \eta}{R T}\right)-\frac{C_{V^{3+}}^{S}}{C_{V^{3+}}} \exp \left(-\frac{\alpha F \eta}{R T}\right)\right]
$$

where $C_{j}^{S}$ is the concentration of species $j$ on the surface of carbon fibres, while $C_{j}$ is the bulk concentration of species $j$.

The overpotential $\eta$ at the electrode-electrolyte interface for the single electron transfer is defined by the Nernst equation [102,103]:

$$
\begin{aligned}
& E_{e q}=E^{0}+\frac{R T}{F} \ln \left(\frac{C_{V^{3+}}^{S}}{C_{V^{2+}}^{S}}\right) \\
& \eta=\phi_{s}-\phi_{e}-E_{e q}
\end{aligned}
$$

where $\phi_{s}$ and $\phi_{e}$ are local potentials for electrode and electrolyte, respectively, and $E^{0}$ is the standard equilibrium electrode potential.

\subsubsection{LIBs}

As discussed in Section 2.4, a conventional LIB electrode consists of electron conducting materials, active materials and polymer binders, forming a tortuous porous structure. 3D microstructure modelling needs to consider charge transfer and transport of $\mathrm{Li}$ ions in the active particles, potential field within the active materials and the electrolyte, as well as the transport of Li ions within the electrolyte. 
The charge balance in the active particles follows the Ohm's law which can be described as Eq. (3.1.1a). The active particles are usually on the order of 1-10 $\mu_{m}$, whereas conductive additives and binders are comparatively much smaller (tens-hundreds $\mathrm{nm}$ ). Due to their small particle size, the conductive additives and binders are often neglected in the electrode structure for modelling transport and electrochemical performance. Recognizing the considerable volume and the important role of conductive additives and binders in the electrode, researchers have tried to include a conductive binder domain (CBD), which treats the conductive additive and binder as a joint phase [77,104-106]. The ion conduction is neglected for $\mathrm{CBD}$ as its conductivity is approximately $5 \%$ of the ionic conductivity of the electrolyte, while the electron conduction in CBD is formulated by Eq. (3.1.1a). One could argue that since the conductive additive particles are not distinguished from the binders, such formulation of electron conduction in CBD may give inaccurate information on the electron transport pathways, therefore leading to inaccurate prediction of electrochemical performance.

The transport of Li ions in active particles is described as:

$$
\frac{\partial c_{1}}{\partial t}+\nabla \cdot\left(-D_{1} \nabla c_{1}\right)=0
$$

where $c_{1}$ is the Li concentration in active particles, $D_{1}$ is the diffusivity of $\mathrm{Li}$ in active particles.

The potential field in electrolyte phase can be obtained as [107]:

$$
\nabla \cdot\left[-\sigma_{i o n} \nabla \phi_{i o n}+\frac{2 \sigma_{i o n} R T}{F c_{2}}\left(1+\frac{\partial \ln f_{2}}{\partial \ln c_{2}}\right)\left(1-t_{+}\right) \nabla c_{2}\right]=0
$$

where $\sigma_{\text {ion }}$ is the electrical conductivity of the ion conductor electrolyte, $\phi_{i o n}$ is the electrical potential, $c_{2}$ is the $\mathrm{Li}$ ion concentration in the electrolyte, $t_{+}$is the transference number of positive ions, $f_{2}$ is the mean molar activity coefficient of the electrolyte.

The mass transport of $\mathrm{Li}$ ions in the electrolyte is expressed as:

$$
\frac{\partial c_{2}}{\partial t}+\nabla \cdot\left[-D_{2}\left(1-\frac{d \ln c_{0}}{d \ln c_{2}}\right) \nabla c_{2}+\frac{J_{2} t_{+}}{F}\right]=0
$$

where $D_{2}$ is the reference Li ion diffusivity in the electrolyte, $d \operatorname{lnc}_{0} / d \operatorname{lnc}_{2}$ is the concentrated solution correction to salt diffusivity, $J_{2}$ is the current density in the electrolyte.

At the active particle-electrolyte interface, the Butler-Volmer equation is used to describe the chemical kinetics: 


$$
N=\frac{i_{0}}{F} \times\left\{\exp \left[\frac{\alpha F \eta}{R T}\right]-\exp \left[\frac{-(1-\alpha) F \eta}{R T}\right]\right\}
$$

where $N$ is the flux of lithium ions, $i_{0}$ is the exchange current density, $\alpha$ is the charge transfer coefficient, $\eta$ is the overpotential at the particle-electrolyte interface. Simple Marcus kinetics have been proposed [108] but not applied so far in 3D microstructure-resolved simulations.

The exchange current density $i_{0}$ is evaluated as a function of the local species concentration in Li-ion battery modelling [32,33,109-111], such as:

$$
i_{0}=k_{0} c_{2}^{\alpha} c_{1}^{\alpha}\left(c_{1}^{\max }-c_{s}\right)^{1-\alpha}
$$

where $c_{2}$ is the $\mathrm{Li}^{+}$concentration in the electrolyte, $c_{1}$ is the Li concentration in the active particle and $c_{1}{ }^{\max }$ the maximum capacity of the material.

The overpotential $\eta$ is given by:

$\eta=\phi_{1}-\phi_{2}-E_{e q}$

where $E_{e q}$ is the open circuit equilibrium potential at the solid/electrolyte interface.

In some cases, as in resistor network models [112,113], the electrochemical kinetic expression is linearized [114-116]. This simplification, totally acceptable for low activation overpotentials, removes a non-linear term in the/governing equations, resulting in a more robust stability of the numerical scheme. Concentration losses are normally considered in Eq. (3.1.4 c) by including the equilibrium potential difference within the definition of the local activation overpotential [92]. For example, in Li-ion batteries the equilibrium reference potential $E_{e q}$ is evaluated as a function of the local state-of-lithiation, normally by using empirical correlations [109,117] based on fitting of discharge curves at low C rates or, more rarely, based on Redlich-Kister expansion [118]. For phaseseparating materials, such as LFP, the kinetics of lithium intercalation must be properly coupled to phase transformation as shown by Bazant and co-workers [119-122].

\subsection{Numerical methods}

Different numerical methods have been employed to solve the transport and reaction processes within 3D porous electrodes. The most popular methods include the finite element method, finite volume method, and lattice Boltzmann method, which are reviewed in this section. All the methods reviewed here can work with both synthetic structures generated in computers and real structures obtained from 
experimental tomography. Pore network models (PNM) have also been used, but mainly for PEMFCs and RFBs, with its own limitation of working with simplified geometry. For this reason, we do not cover the numerical details of the PNM method here, but rather discuss their applications in Section 4.

\subsubsection{Finite element method (FEM)}

The FEM is a numerical method used for solving systems of partial differential equations (PDEs) by recasting them as a system of algebraic equations through the discretisation of the continuum problem over a finite number of nodes and elements, which constitute the computational mesh. Generally speaking, the FEM can be regarded as the evolution of the finite difference method (FDM), which is still used in 3D modelling for the efficient evaluation of effective properties [57], for electrochemical impedance spectroscopy (EIS) diffusion simulations [123,124] and multi-scale modelling [125]. For a general introduction to FEM, the reader is referred to specific textbooks [126]. This section reviews the application of FEM to 3D electrode microstructure modelling, focusing the attention on the efforts made in producing an accurate mesh, coupling different physics and applying specific strategies to help model convergence to a solution.

The popularity of FEM in the electrochemical energy community has been boosted by the widespread use of commercial software, among which COMSOL Multiphysics [127] is probably the most popular one for electrochemistry [33,98,111,114,116,128-137]. Nonetheless, open-source codes based on deal.ii library [138], such as OpenFCST [99] and ParCell3D [115,139], Sandia's Sierra Module Aria [78,140-143] or NIST Object Oriented Finite Element Analysis program [144] are used as well, while in-house FEM implementations are mainly used to overcome specific limitations of available codes [118].

One of the challenging tasks in using FEM is the production of a satisfactory computational mesh, normally based on tetrahedral [114,118,132,140-143] or tetragonal elements [145]. While the problem is not dramatic in studies which exploit symmetry axes [146], performed in 2D slices $[32,112,133,135,144]$ or in regular 3D patterns [114], meshing a complex multiphase 3D microstructure is typically quite demanding as the number of mesh elements ranges from one million or less $[111,118,145]$ up to tens of million $[115,132,139]$, or even more than one hundred million [142], requiring up to one TB of memory. Normally, specific meshing software is used, such as commercial Simpleware ScanIP [132], the XWind extension of Amira-Avizo [147], open-source iso2mesh [98,131], and TetWild [148], while using voxels as a node in the FE mesh is rarely done [99]. Maintaining high fidelity to real microstructure shapes while minimising mesh elements for 
computational speed, while also maintaining computational accuracy of the solutions reached is a considerable challenge. Significant progress has been made by Roberts and co-workers [78,140143,149], who use a conformal decomposition FEM (CDFEM) scheme by first generating a tetrahedral background mesh, which is then decomposed into smaller elements at particle/electrolyte interfaces to ensure a conformal mesh (Figure 6). A solution verification of CDFEM on mesh size was performed first by Roberts et al. [140] by investigating the convergence of five quantities of interest for different mesh resolutions and, more extensively, in another study on both synthetic and tomography-derived microstructures [149]. Notably, ensuring a conformal mesh is even more critical for reactions taking place at the three-phase boundary, as in SOFCs [98].
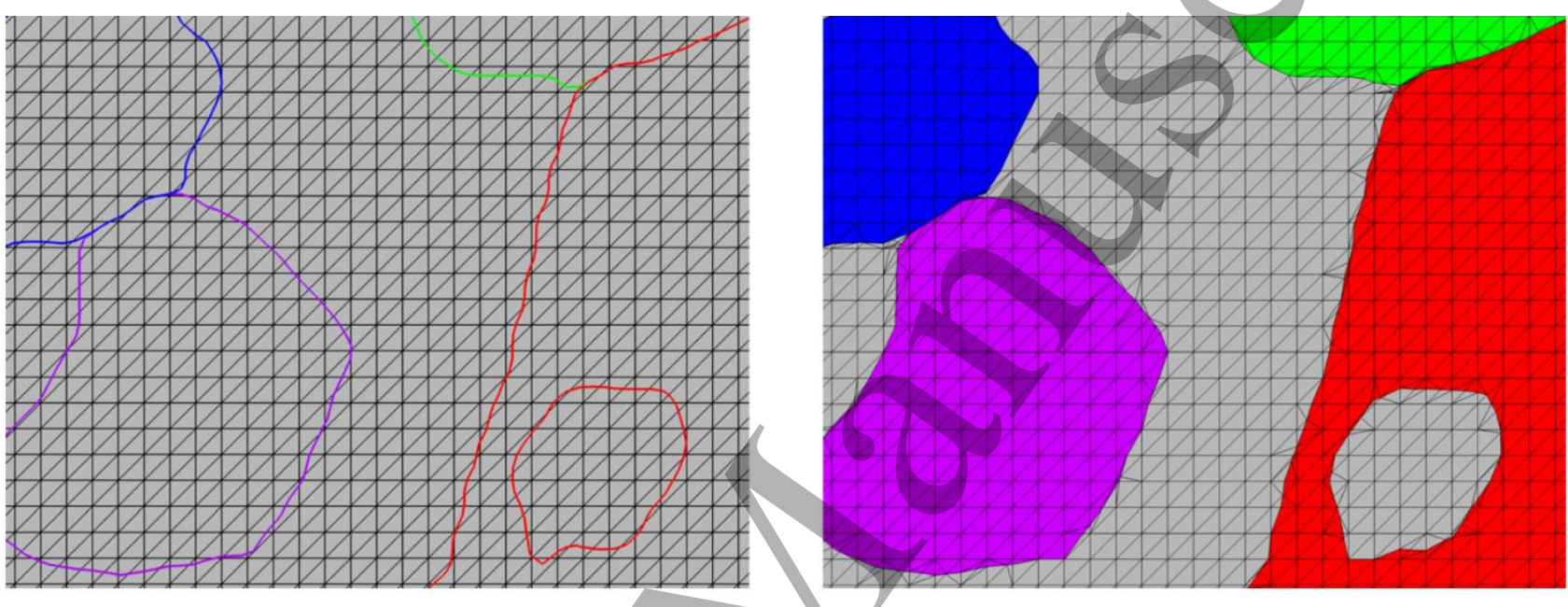

Figure 6: Illustration of the CDFEM process for the reconstruction of LIB electrode microstructure, visualised on a two-dimensional plane: first a tetráhedral background mesh is generated over the entire domain using linear (Q1) basis functions, then the elements that contain facets are automatically decomposed into smaller elements to enforce/mesh conformity [reproduced from [140]]

An alternative approach to address the challenges described has been comprehensively described by Fang et al. [118,150], who introduced a mortar-based finite element approach for 3D modelling of Li-ion battery electrodes. Instead of relying on conformal mesh at the particle/electrolyte interface, which requires strong pointwise constraints on fluxes, they apply a weak imposition of the interfacial constraints, namely, the Butler-Volmer kinetics (which, in mathematical terminology, is a Robin-type interfacial condition). This allows flexible and independent grid generation on the two domains: a finer mesh in the solid domain, where strong interfacial gradients in Li concentration are expected due to its low solid diffusivity, and a coarser mesh in the electrolyte domain, where Butler-Volmer interface integrals are evaluated and then fluxes are projected onto the particle side through a discrete interface mapping. Fang et al. [118] tested the mortar-based approach up to an interfacial node number ratio of $2.5: 1$, thus cutting the overall mesh requirements by one third, showing no numerical 
perturbations at the solid/electrolyte interface and resulting in spatial convergence in the same fashion of conventional matching-node coupling [118].

FEM has been extensively used to solve just a single physics, for example for the evaluation of effective transport properties [110,134,142,151-153] or for structural mechanics [145,154] simulations, sometimes coupling both these aspects [78]. For these applications, FEM is currently more practical than the resistor network approach [112,113,155-157]. Nevertheless, FEM is useful when more physics are simulated together, such as different transport mechanisms occurring in different domains coupled by interfacial (or TPB) charge-transfer kinetics. This is the standard problem simulated in all the microstructure-resolved 3D electrochemical models $[98,99,115,116,118,128,132,136,137,139]$. Conversely, multiple parallel reaction pathways are seldom considered. Especially in LIBs, more physics are simulated, such as Li diffusion and structural mechanics in electrode particles, to take into account the stress generated by Li intercalation and, in turn, the effect of mechanical stress on Li diffusivity $[33,107,141,143]$. Similarly, heat balance is sometimes coupled with the electrochemical model [32,140], so that the temperature field is solved on the same computational mesh used to solve for species concentrations and electric potentials. This normally means that, in the same domain, the mesh requirements are dictated by the physics which requires a finer mesh to resolve stronger gradients, leading to an unnecessary increase in degrees of freedom for the processes which do not need accurate spatial discretisation. Although FEM has been used to simulate single phase transport within porous electrode microstructures, it faces challenges to track the complex interface between two phases (gas and liquid) in the porous electrode [158], which means it may be unsuitable for simulating the gas-liquid two phase flow in PEMFCs.

This problem is basically the same one arising when simulating phenomena taking place at different length scales, which is typically overcome by using a multi-scale approach [15]. An interesting approach in this regard was presented by Lee et al. [111], who proposed a variational multi-scale enrichment method (VMS). Unlike conventional approaches based on homogenisation, the VMS method decomposes the different scales without assuming scale separation. In order to model microscopic details, the domain is partitioned into coarse and fine mesh domains. Notably, the fine domain is not a point in the coarse domain; rather, it is a portion of the coarse domain so that the multi-scaled portion of the coarse domain coincides with its companion microscopic model. In the specific case, the Li concentration in electrode particles remains in the fine domain and is not multiscaled, while the $\mathrm{Li}^{+}$concentration and electric potentials are multi-scaled in coarse and fine components. Taking advantage of a weak integral FE formulation of the governing equations, coupled 
variables from coarse and fine domains appear as force terms, while at the fine scale, the coarse domain variables are assumed to be homogenised. This approach speeds up the solution compared to direct numerical simulations [111].

An even more critical problem arises when large domain deformations are expected, such as when simulating the swelling of silicon particles upon Li intercalation in Li-ion negative electrodes. As an example, Wang et al. [133] reported a coupled electrochemical/mechanical model in which both submodels share the same FE mesh and are coupled through the deformation fields and Li concentration. The large mechanical deformation of silicon particles is simulated according to the finite strain theory, in the material coordinate system. However, mass conservation is not automatically satisfied in the electrochemical sub-model, because Li flux at the silicon/electrolyte interface does not account for particle deformation. In order to circumvent the problem, a correction coefficient at the silicon/electrolyte boundary is introduced by Wang et al. [133] to account for volume expansion. This appears to be only a precursory way to address the issue. The problem of moving interfaces (and also phase transition materials, e.g., $\mathrm{Li}_{x} \mathrm{FePO}_{4}$ in Li-ion batteries) might be more conveniently tackled by using adaptative mesh refinement [159], arbitrary lagrangian-eulerian methods [160] or by phase field approach [146], which are currently seldomly uséd in multi-physics microstructure-resolved models.

The numerical schemes adopted to solve non-linear equations are crucial. A Newton-Raphson iteration scheme [99,111,115,118,140-143] with proper termination criteria [99] is typically used. Direct solvers of the (sparse) linear systems, like MUMPS [99], are used only for single-physics simulations because of their high memory requirements in multi-physics 3D models [115]. Iterative solvers [142] of the linear system, such as GMRES [78,114,115], are more often used. However, since the problem is ill conditioned [115,118], a preconditioner is normally applied, such as ILU [115], multi-grid/multi-level [78,118] or Jacobi [142] preconditioner. Segregated (partitioned) numerical schemes [143], which reduce memory requirements, can be used only when there is weak coupling between different physics, otherwise all the physics must be solved together in a monolithic fashion [118].

Transient simulations are needed in battery models, to capture the dynamic events. Such simulations require time integration, for which second order back differentiation (BDF2) [33,111,143,161] is considered more stable than explicit schemes [123,125,144]. Notably, it is computationally intensive to simulate electrochemical impedance spectroscopy (EIS) with time, following Fourier transformation of the oscillating response $[116,129]$. Therefore, recasting equations in the frequency 
space and solving the resulting steady-state problem [98,123,124] should be preferred in microstructure-resolved simulations.

\subsubsection{Finite volume method (FVM)}

FVM shares many similarities with FEM. In fact, at first sight, FVM may be loosely regarded as a special case of the FEM weak formulation with a constant test function. In addition, as in FEM, the FVM also requires a computational mesh to discretise the PDEs in algebraic form. Nevertheless, there are some remarkable differences between FEM and FVM. The main differences are that, instead of using elements and test functions as in FEM, FVM works on a finite number of control volumes, which are the cells of the computational mesh. In FVM, conservation equations are integrated in each cell as if each control volume were an individual domain: this ensures a stricter local conservation (of mass, energy, momentum) while the FEM guarantees only a global conservation. As such, flux terms, which appear within a divergence operator in the PDEs, are converted to surface integrals in the FVM according to the divergence theorem. Then, while in FEM the implementation of boundary conditions is relatively straightforward, ghost cells are required in the FVM to fictitiously extend the computational domain to add the boundary conditions. The final main notable difference between FVM and FEM is in the numerical stabilisation of convection-dominated fluxes, which is dealt more easily in the FVM. However, both FVM and FEM have advantages and drawbacks, so there is not a general rule to select which method may perform better for the specific application. Further details of the FVM formulation can be found in specific textbooks $[162,163]$.

In the context of microstructure-resolved 3D modelling of electrochemical devices, FVM has been applied less frequently than FEM. In the majority of cases, an in-house FVM implementation is used [76,77,88,89,164-169] but open-source [170] and commercial [37,109,170-172] software is applied as well.

The computational mesh for FVM is typically made by regular cuboid [88] and structured hexahedral cells [165,166,168,169], but also polygonal meshes are used [172]. Regular grids are used especially for the volume of fluid (VOF) approach $[89,167,173]$ and in the sub-grid scale modelling approach $[165,166,168,169]$ (Figure 7). In both VOF and sub-grid scale modelling approaches the local microstructural details are not explicitly resolved but are effectively considered via averaged properties within each cell, resulting in a local continuum approximation of the $3 \mathrm{D}$ microstructure at a length scale which is larger than the microscopic feature size but smaller than the electrode thickness. These approaches allow for a substantial reduction in the number of degrees of freedom, 
being more effective than voxel resampling [168]. On the other hand, even in FVM, when local structural details and sharp interfaces are explicitly resolved [76,77,109], i.e., by using the same meshing approach extensively discussed in the previous section, special attention is paid to refine the mesh where required, for example refining a cut-cell mesh at surfaces [37] and TPBs [170]. A specific analysis on the number of finite volume edges bordering three phases and the errors introduced by the voxel-based representation of TPBs is reported by Prokop et al. [88].
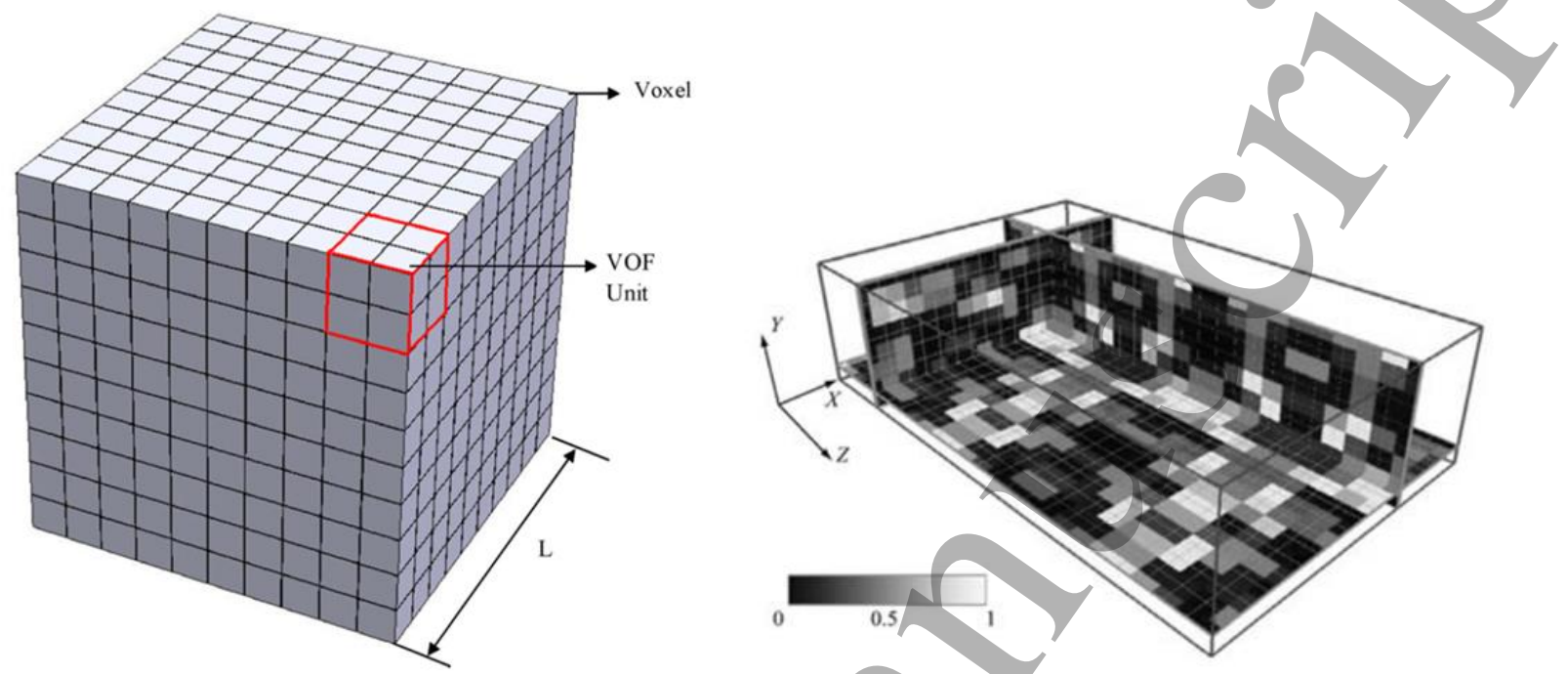

Figure 7: a) Typical cuboid mesh used in the VOF FVM approach (reproduced FVM examples from Fig. 2 of ref [167]); b) distribution of Ni volume fraction in an SOFC anode in the grid used by the sub-grid scale modelling approach (reproduced from ref [168]).

The numerical schemes used to compute the solution are rather similar to the FEM ones. NewtonRaphson [172,174,175] and Picard outer-iteration [37] schemes with successive relaxation [37,88] are used to iteratively solve the non-linear systems, sometimes by computing the Jacobian matrix analytically [172]. At each iteration the resulting linear system is solved with iterative methods, such as GMRES with localised ILU [174,175] or multigrid [37,172] preconditioners. For weakly coupled physics, segregated solvers can be used [37]. For time-dependent models, as in Li-ion batteries, the second-order FV discretisation is often coupled with an implicit second-order algorithm for marching in time [37].

Similar to FEM, FVM faces challenges to track the complex interface between two phases (gas and liquid) in the porous electrode [158], making it unsuitable for simulating the gas-liquid two phase flow in PEMFCs.

\subsubsection{Lattice Boltzmann method (LBM)}


The LBM is a kinetic theory-based numerical technique for solving transport problems. A lattice model is uniquely identified by the number of dimensions D and by the number of speeds Q. Currently, the most employed model for two dimensional simulations is the D2Q9, while for three dimensional ones, models with D3Q7, D3Q15, D3Q19 and D3Q27 [176] are available.

Unlike conventional computational fluid dynamics methods, which are based on the discretization of macroscopic governing equations, the LBM method is based on microscopic models and mesoscopic kinetic equations, in which the collective behavior of the particle distribution function is used to simulate the continuum mechanics of the system[177]. Also, for multiphase flows, the interface between different phases is automatically maintained, hence its reconstruction or tracking is avoided [158]. So, there are some native advantages for LBM simulations. It is easy to apply for complex domains (flows in porous media), easy to handle complex flows such as multi-phase and multicomponent flows without needing to trace the interfaces between different phases. In addition, it is easy to adapt to parallel processing computing. In the lattice Bhatnagar-Gross-Krook (BGK), the collision operator is based on the single-relaxation-time approximation to the local equilibrium distribution [178]. In addition, the numerical stability of LBM,/especially in the high-Reynolds number simulations was increased by the multi-relaxation time (MRT) lattice-Boltzmann model.

LBM has received significant attention in modeling multiphase flow and transport in complex porous microstructures, and has proved to be useful in simulating gas-liquid two phase flow in PEMFC electrodes [67,68,179] and RFB electrodes [31,69]. For this reason, LBM have been adopted to study the water distribution and water evolution in the GDL [68].

In the LBM model, the two-phase flow can be expressed as [68]:

$$
f_{\alpha}\left(\boldsymbol{x}+\boldsymbol{e}_{\alpha} \delta t, t+\delta t\right)=f_{\alpha}(\boldsymbol{x}, t)-\frac{1}{\tau}\left[f_{\alpha}(\boldsymbol{x}, t)-f_{\alpha}^{e q}(\boldsymbol{x}, t)\right]+\boldsymbol{F}_{\alpha}
$$

where $f_{a}$ is the particle distribution along the $\alpha_{\mathrm{th}}$ direction and $f_{\alpha}^{e q}$ is the equilibrium distribution, $\delta \mathrm{t}$ is the time step, $e_{a}$ is the particle velocity in the $\alpha_{\mathrm{th}}$ direction, and $\tau$ is the single relaxation time. The total force on each particle $F=F_{1}+F_{2}+F_{3}$ includes the fluid-fluid cohesion $F_{1}$, the fluid-solid adhesion force $F_{2}$ and body force $F_{3}$.

LBM is also well suited for solving the convection-diffusion equation (3.1.3a) for describing the liquid electrolyte flow in RFBs [31,69]. Although in principle, FEM and FVM would be possible to solve the convection-diffusion equation, there have not been such studies in previous publications, at least for microstructure-resolved electrochemical systems. 
Although LBM proves to be a promising approach, as an emerging method, there are currently no established commercial or open-source codes based on LBM to incorporate multi-physics interactions such as transport of species with charge transfer/electrochemical reactions in electrochemical electrodes. As a result, the LBM method has not been as widely used for the electrochemical energy devices as FEM and FVM. Nonetheless, a few research groups including the authors [30,31,68,69,101,180-184] have made considerable progress in developing LBM/based modeling frameworks with the capability of simulating multiphase flow and charge transfer process, for fuel cells and batteries. More details will be provided in Section 4.

\section{Applications of 3D microstructure modelling in different electrochemical energy devices}

\subsection{Solid oxide fuel cells (SOFCs)}

A popular use of 3D microstructure modelling in SOFCs consists of the evaluation of effective transport properties, such as the tortuosity and the constriction factors $[58,152,185,186]$, by solving the Laplace equation $\nabla^{2} c=0$ within the 3D domain of the selected solid or porous phase $[187,188]$, while paying special attention to the representativeness of the domain [189-191], which is either reconstructed from tomographic datasets or synthetically generated via packing algorithms. In this regard, the open-source application TauFactor [57] is increasingly employed in the field, being as easy to use and fast as resistor network approaches [192,193]. Similarly plugins for these calculations have also become available in ImageJ and in commercial software such as FEI Avizo [194]. However, grain boundary and interfacial resistances in ohmic transport are only rarely considered $[123,191]$.

Similarly, microstructure-resolved solid mechanics FEM simulations have been increasingly used to determine the effective elastic modulus and Poisson ratio of SOFC electrodes [145,154]. These have been directly correlated with nano-indentation and cracking experiments [195], and effects on phases in composite electrodes [196]. Thermo-mechanical equations have been recently coupled with complete electrochemical modelling comprising balance of charge, species, heat, and momentum, as well as Butler-Volmer kinetics in Ni-YSZ cermets by Celik et al. [135]. Although the study is performed only on a representative 2D slice, the model predicts high stress values at Ni grains upon heating, which may result in micro-cracks (Figure 8). Cracks are also detected upon anode reoxidation [197]. Nakajo et al. [193], by using an electrochemical fin model which represents the microstructure as a network of axisymmetric fins, identify a significant increase of ca. $12 \%$ in area-specific 
resistance upon cracking of the YSZ framework, highlighting that the standard topological metrics (e.g., mean particle size, geometric tortuosity) are not sensitive enough to identify cracks.
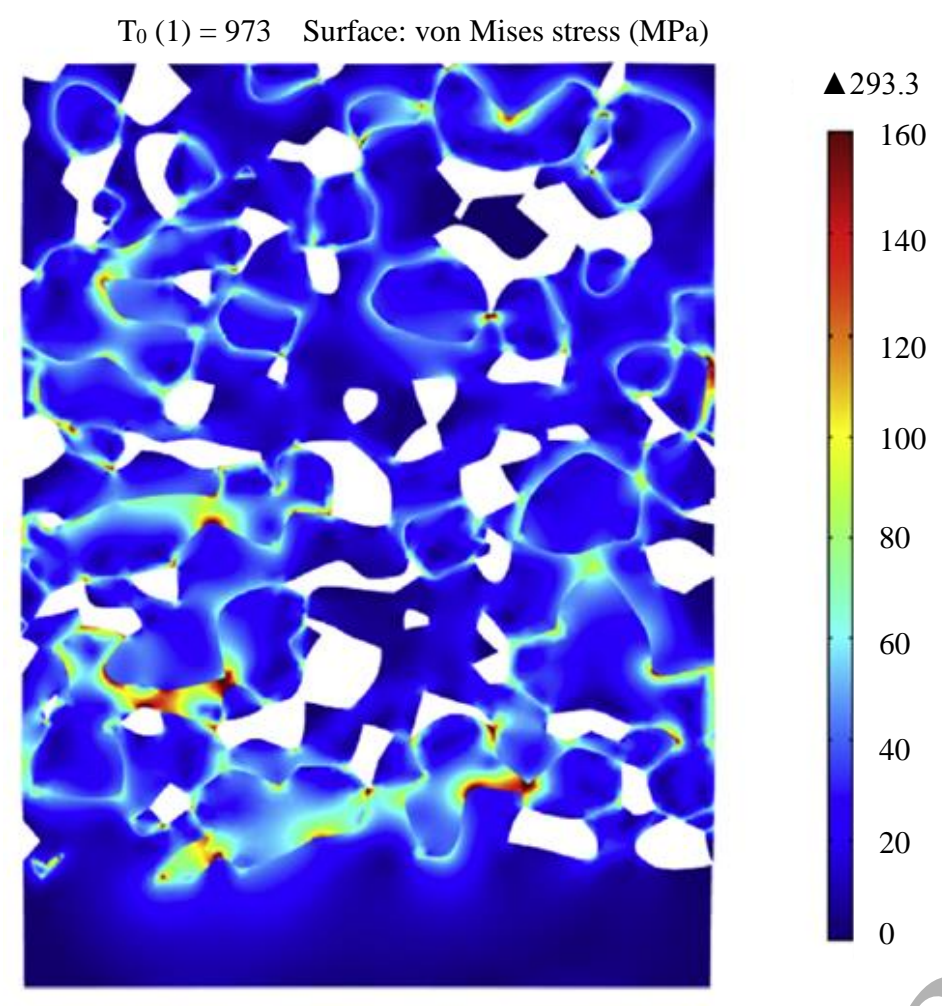

Figure 8: The stress distribution simulated by a thermo-mechanical model in a 2D slice of a Ni-YSZ anode, showing stress concentration at the Ni grains. (reproduced from [135])

In recent years, several research groups have developed microstructure-resolved models focusing mainly on solving for species and charge transport within 3D electrode microstructures in order to guide electrode design. It was found that modern SOFC electrode scaffolds are good structures for performance [21]. In addition, as a rapid, yet detailed, microstructure performance assessment tool, resistor network [157,198] and electrochemical fin models [131,199] have been consistently applied to understand transport behaviour in electrodes. Schneider et al. [157] investigated the effect of the volumetric fraction of solid phases in synthetic microstructures, using a resistor network model, suggesting that the lowest polarisation resistance is expected for a composition close to the percolation threshold of the electron-conducting phase. Similar conclusions were reached by Gawel et al. [170] for computer-generated Ni/YSZ anodes using FVM and taking into account gas concentration losses, predicting a maximum current production for a 40\%/60\% Ni/YSZ composition with $15 \%$ porosity. These indications were verified by Kishimoto et al. [169], who tested, reconstructed and modelled Ni/YSZ anodes with different compositions, highlighting the importance of Ni percolation and active TPB density distribution along the thickness. Electronic and ionic percolation are improved by grading the composition along the electrode thickness, i.e., by using a larger volume fraction of the ion-conducting phase close to the electrolyte interface and linearly 
reducing it in favour of the electron-conducting phase close to the current collector, as investigated by Schneider et al. [198]. Nevertheless, simulations show that the graded electrode does not perform significantly better than a composite electrode with optimized uniform composition [198] or indeed a scaffold structure [21]. Lynch et al. [98] simulated the bulk and surface pathways of the oxygen reduction reaction in LSM-YSZ cathodes by using the FEM. EIS simulations reveal that the surface reaction path is dominant at low overpotentials, in fair agreement with continuum modelling predictions [200-202], suggesting that nanostructured LSM networks near the TPB region may provide increased surface area and thus boost the cathode performance. The role of the electrode thickness was investigated via FVM by Cai et al. [167] and Miyawaki et al. [165], who predict an active thickness in the order of 5-15 $\mu \mathrm{m}$ for Ni-YSZ anodes and provide simple analytical expressions for its estimation. Finally, the optimal sintering temperature of Ni/YSZ anodes was investigated by Suzue et al. [87] by comparing experimental ASR values with LBM predictions. On the other hand, physically-based 3D microstructure-resolved simulations have been rarely used to assess the impact of degradation phenomena, for example by integrating phase field modelling of nickel coarsening with a LBM solving for species transport and electrochemistry as shown by Jiao et al. [203].

Special attention has been paid to compare model predictions with experimental data, mostly in the form of polarisation curves (i.e., I-V curves) or polarisation resistance measured via EIS. In fact, several models have been properly validated against experimental data in different operating conditions $[89,95,165]$. Very often the exchange current density is adjusted to fit experimental data [86,89] or different exchange current density models are compared [85].

3D microstructure-resolved models are also often compared against predictions made by wellestablished 1D continuum models in order to validate the framework [88,132,165]. A comprehensive investigation in this sense was made by Ivers-Tiffée and co-workers [115,116,139]. Microstructureresolved simulations of a LSCF cathode, comprising gas diffusion, oxygen ion transport and surface exchange reaction, were compared with the well-known Adler-Lane-Steele (ALS) continuum model [204], showing good agreement of macroscopic fields, such as the current density as a function of the distance from electrolyte, and only $5 \%$ error in ASR predictions [115,139]. The major discrepancy, in the order of $12 \%$, arises in the prediction of the penetration depth as a consequence of the approximation of the current decay and the averaging method of the ALS model. A good agreement between microstructure-resolved and continuum models was also proved in EIS simulations [116], giving a comprehensive explanation of the origin of the Gerischer impedance response of MIEC cathodes. Kreller et al. [129] elaborate further on the EIS response showing that, when the electrochemically-active thickness is significantly larger than the particle size, the $1 \mathrm{D}$ continuum 
approximation is sufficient for an accurate prediction of the impedance response. Only when the particle size is comparable to the active thickness (though this is rarely the case), the threedimensional microstructural details at the electrolyte interface are required for an accurate EIS simulation, suggesting that a hybrid FEM approach (Figure 9), comprising microstructure-resolved simulation at the electrolyte interface coupled with a continuum 1D model beyond a certain cut-off distance, can be used to reduce the computational effort [130]. The effect of microstructure heterogeneity on the EIS response was also discussed by Cooper et al. [124] by considering only Fickian diffusion. In summary, these studies confirm that 1D continuum models provide accurate macroscopic predictions for homogeneous electrode microstructures in typical operating conditions.

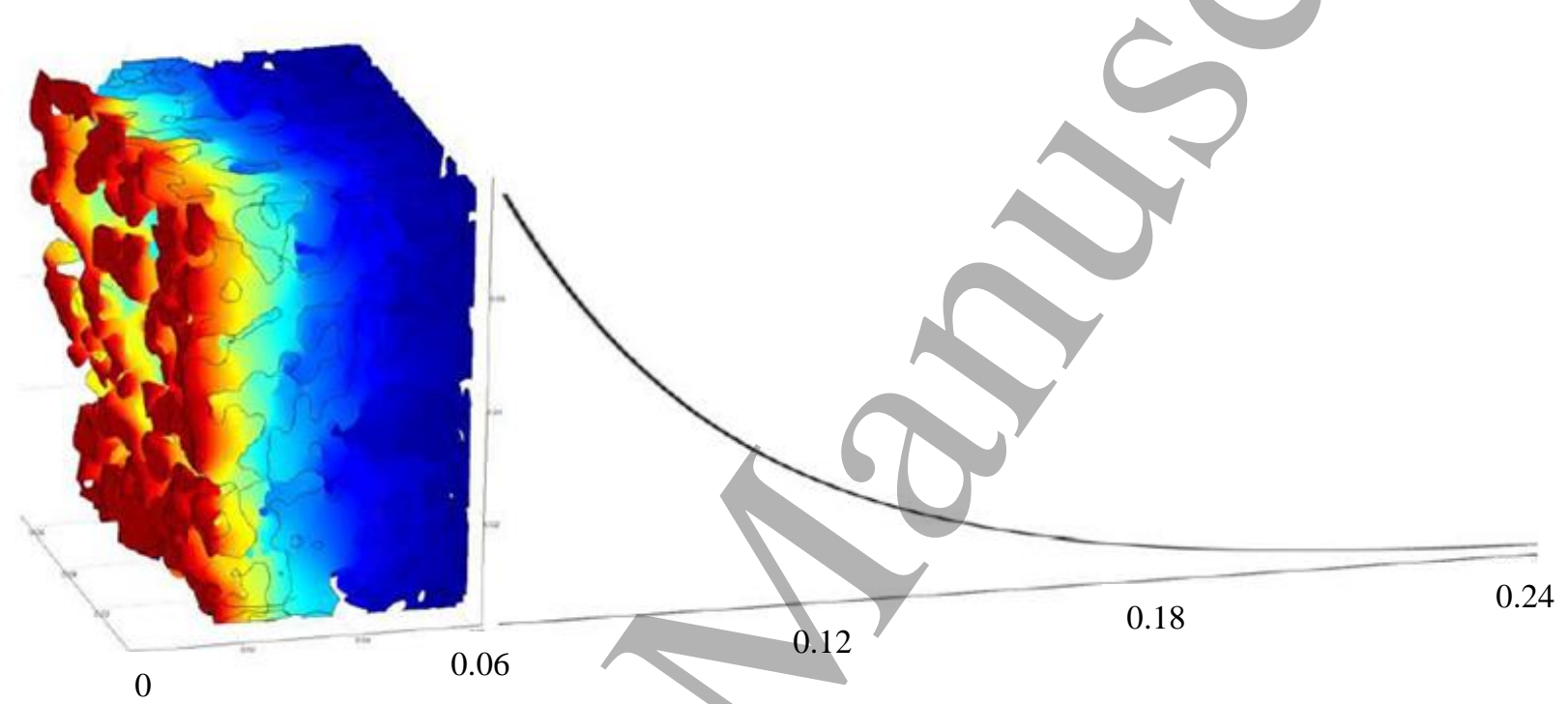

Figure 9: Representation of the hybrid FEM approach proposed in Ref. [130], showing that a 3Dresolved simulation is required at the electrolyte interface while the numerical solution of macroscopic fields can be prolongated by using a continuum 1D model beyond a certain cut-off distance. (reproduced from [130])

On the other hand, such a satisfactory agreement supports the fact that the macroscopic behaviour of SOFC electrodes can be predicted with high fidelity by using continuum models. In other words, provided that the microstructure is homogeneous, and a right set of parameters are obtainable, continuum models can be useful enough, to assess the effect of electrode properties on the global electrode response. Such continuum models are able to predict the polarisation curve, impedance response and even the average voltage and current profiles along the electrode thickness. 

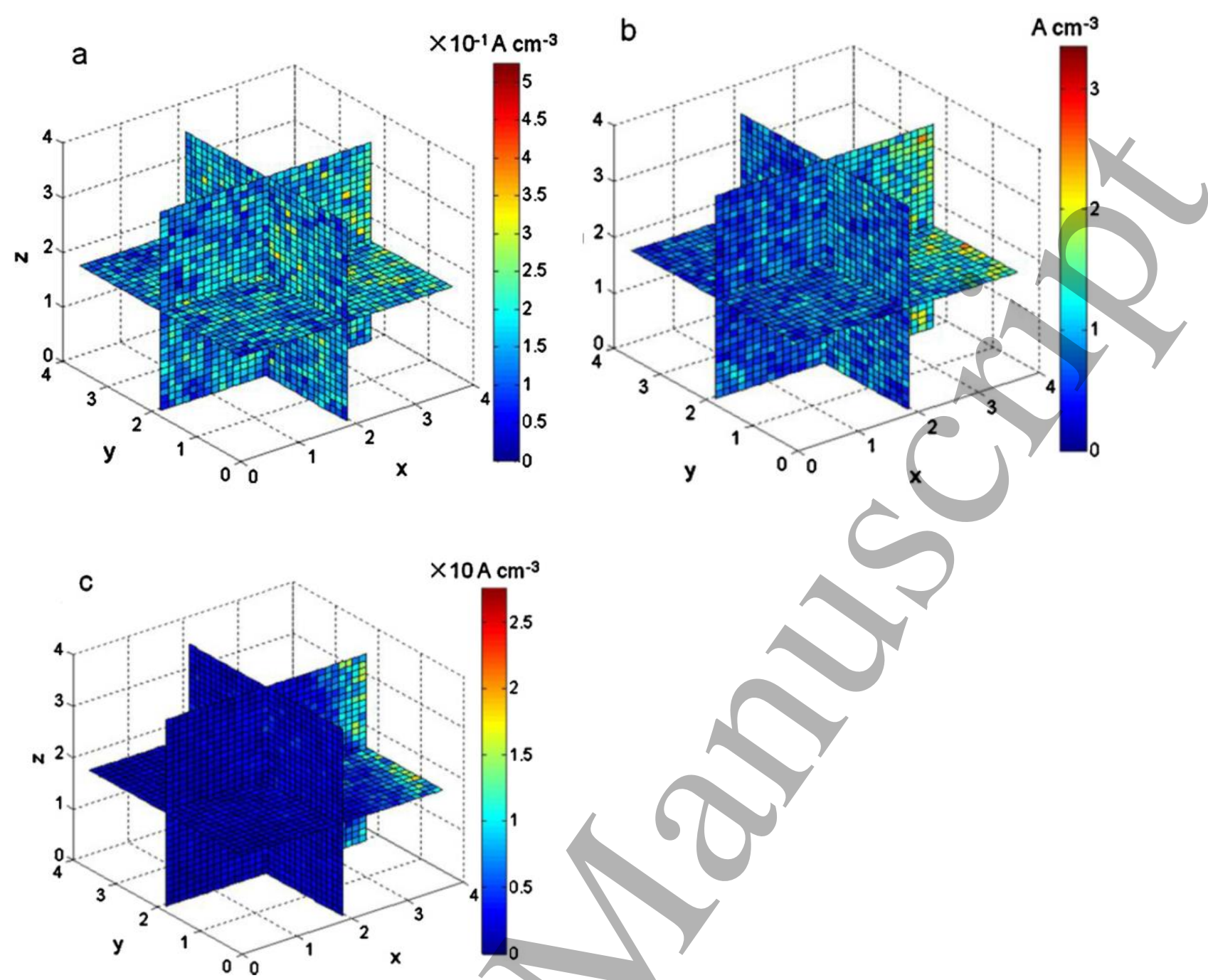

Figure 10. The 3D map of current density distribution in the electrode when over-potential is (a) 50 $\mathrm{mV}$, (b) $200 \mathrm{mV}$, and (c) $400 \mathrm{mV}$ (reproduced from [29]). At $\mathrm{x}=0$ is the interface of current collector with the electrode, and at $\mathrm{x}=3.75$ is the interface of the electrode with the electrolyte.

Therefore, microstructure-resolved 3D models should be used to go beyond such macroscopic predictions and provide detailed quantitative information that macro-homogeneous models cannot resolve [132]. As an example, Prokop et al. [88] investigated the effect of a microstructure heterogeneity by artificially adding a disturbance (i.e., a sphere of pore, conducting or inert phase spanning two thirds of the domain width) to the Ni/YSZ anode microstructure reconstructed with FIB-SEM tomography. FVM simulations show that major discrepancies compared to the homogeneous case arise when the disturbance is placed close to the electrolyte interface, that is, within the active region of the electrode. Heterogeneous current distribution across the electrode was predicted by Cai et al. [29] in 3D microstructures of an SOFC anode (Figure 10), with higher current generation found at the electrode-electrolyte interface when operating at high overpotential. Recently, 
Bertei et al. [132] proposed a novel approach to quantify inhomogeneous current distribution at the particle level by applying microstructure-resolved 3D electrochemical FEM modelling and computing statistics of the local current density experienced by each individual particle. The study reveals that there is a wide scattering of current distribution at the particle level in homogeneous random microstructures, indicating that hot spots may locally arise and that a large fraction of particles (up to $30 \%$ ) is underutilized, which suggests that alternative strategies for microstructure optimisation should be considered (Figure 11). Importantly for the first time the work opens the possibility to predict behaviour, and design electrodes for fabrication, according to their microstructure. Thus, simulation and quantification of local heterogeneity is still at an early stage in the SOFC field. Only recently advanced microstructural descriptors, such as the tortuosity distribution of conducting pathways [205] and the accessible TPB [206], have been proposed. These new metrics enable the quantification of the non-uniform utilization of the electrode microstructure or the heterogeneous distributions caused by degradation phenomena, such as nickel depletion at the electrolyte interface, and loss in connected TPB density as a consequence of Ni coarsening after longterm SOEC and SOFC operation [207], respectively. Investigation of the non-uniform utilization of the electrode microstructure is probably the next direction for SOFC 3D electrode modelling.

a)

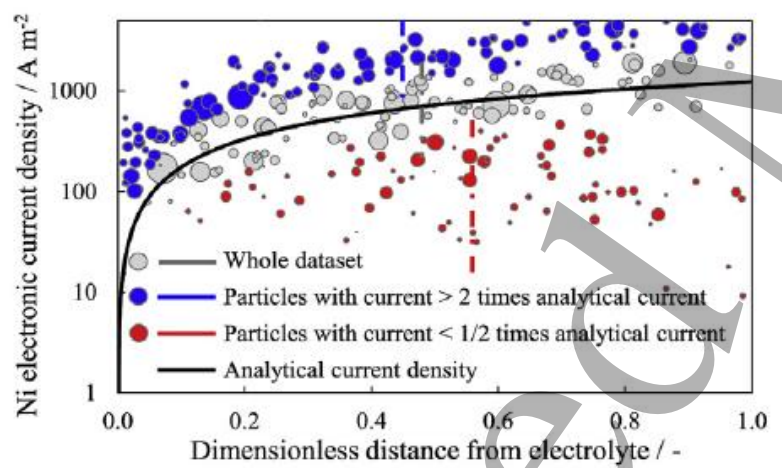

c)

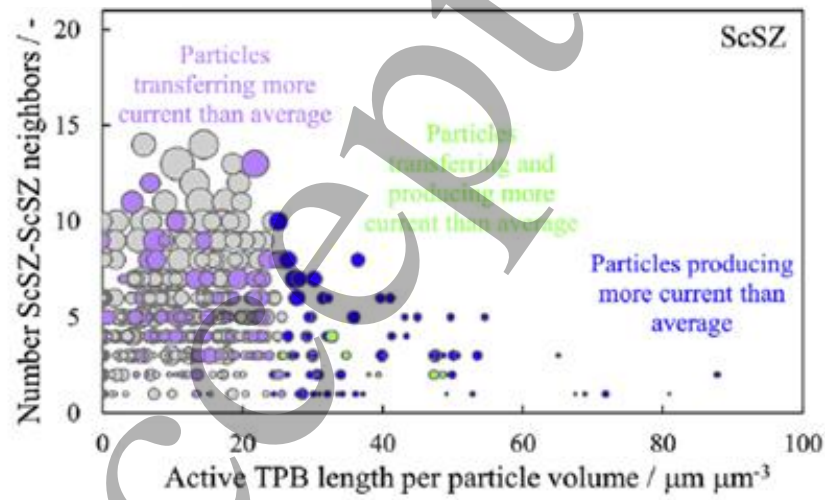

b)

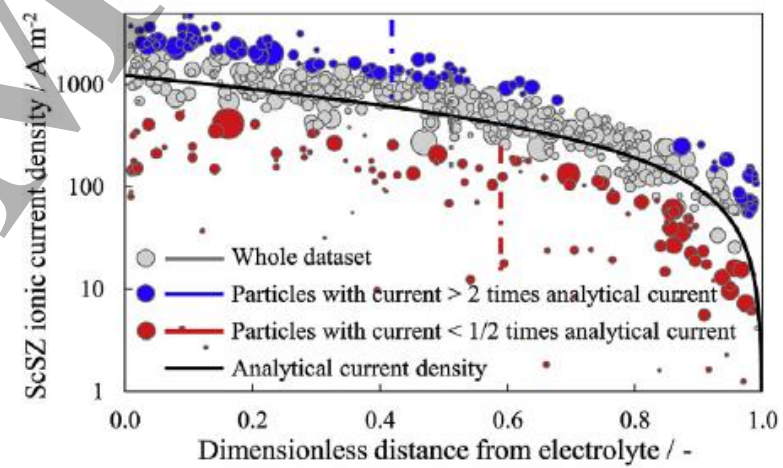

Figure 11: The novel approach proposed by Bertei et al. [132] to quantify inhomogeneous current distribution at the particle level. Current density as a function of the distance from the electrolyte for 
Ni particles (a) and ScSZ particles (b). c) Classification of ion-conducting particles as a function of their connectivity and TPB length, showing that particles which excel in both conducting and producing current (in green) represent only a tiny fraction. In all these plots, each bubble represents an individual particle, whose volume is proportional to the bubble area.

\subsection{Proton exchange membrane fuel cells (PEMFCs)}

Numerical models are required both at the macro- and micro-scales to understand water transport in PEMFCs, to develop improved water and heat management strategies, and design advanced GDL, MPL and CL micro structures. Water management is a crucial problem for efficient operation of PEMFCs. It is essential to understand the liquid water behavior in cathode electrode and its effect on the performance. There are two major approaches based on the macro-continuum methods to model the liquid water distribution in the gas diffusion media (GDM) (which includes GDL and MPL) and CL of PEMFCs, i.e. two-fluid model [23,208-214] and multiphase mixture model [215-220]. By using these two macro-continuum models, it was revealed that the capillary pressure and relative permeability have significant impact on the distribution of liquid water saturation and transport, and relative permeability has even more impact on the liquid water transport than the capillary pressure [213]. Moreover, the transport and reaction processes in the CL have been studied, and it was found that the cathode performance is dependent on the Nafion and platinum loadings and distributions [221]. However, the continuum macro models have several limitations when considering micro-scale phenomena during liquid water and species transport in porous media because the accurate prediction is dependent on the correct liquid and species transport properties, such as capillary relationship, permeability and effective diffusivity and so on.

Microstructure-resolved (or pore-scale) modeling of PEMFC electrodes solves the equations describing gas transport, charged particle conduction, heat transfer, and the electrochemical reactions on the $3 \mathrm{D}$ porous microstructure of electrodes, so that the effects of microstructures are explicitly considered. As for the numerical schemes, the governing equations are typically solved by FVM [174,175,222-225], FEM [99,226-229] or finite difference method (FDM) [125] approaches when transport in the pores considers only the gas phase. Singh et al. [222] constructed the CL structure of the PEMFC electrode by using tomography, and simulated values of the effective oxygen and water vapor diffusivity, proton and electron conductivity, and thermal conductivity using FVM. The simulated values were in reasonable agreement with measured data from experiments reported in the literature. Lange et al. [175] computed the effective transport properties of the CL over a range of operating conditions including the effective water vapor diffusivity, effective oxygen diffusivity, effective proton conductivity, effective electron conductivity and the effective thermal conductivity. 
Recently, the effect of porosity and water saturation on the oxygen diffusivity in the CL was investigated using FVM to solve the governing equations for immiscible, two-phase flow [223]. The liquid water and oxygen concentration distribution in the CL is shown in Figure 12. In order to explore the wettability effects, simulations were performed using different contact angles to represent the hydrophilic and hydrophobic [224] properties of the surface. Results indicate hydrophilic surface gives larger diffusive transport values compared to the hydrophobic media where the liquid water preferentially floods the larger pores. Cetinbas et al. [226] developed an agglomerate model employing a distribution of discrete catalyst particles within the agglomerate to investigate particlelevel diffusion effects based on FEM. Then, with the same method, the effects of Pt and ionomer loading were investigated and the results were found to be in excellent agreement with experiments [227]. In order to accelerate pore-scale simulation of the CL, Zheng et al. [125] combined the macroscopic approach with pore-scale simulation by decomposing a physical quantity into macroscopic and local variations. It was shown that this multiscale scheme reduces the computational cost without sacrificing accuracy.

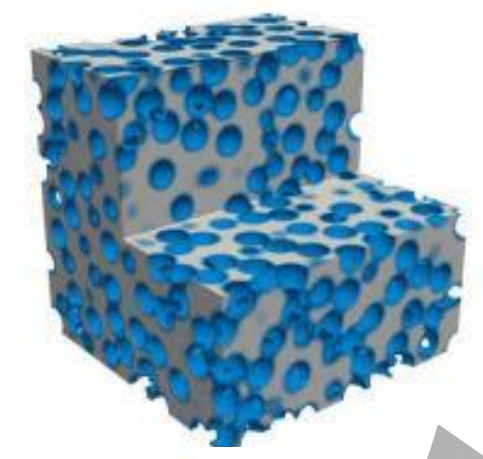

(a)

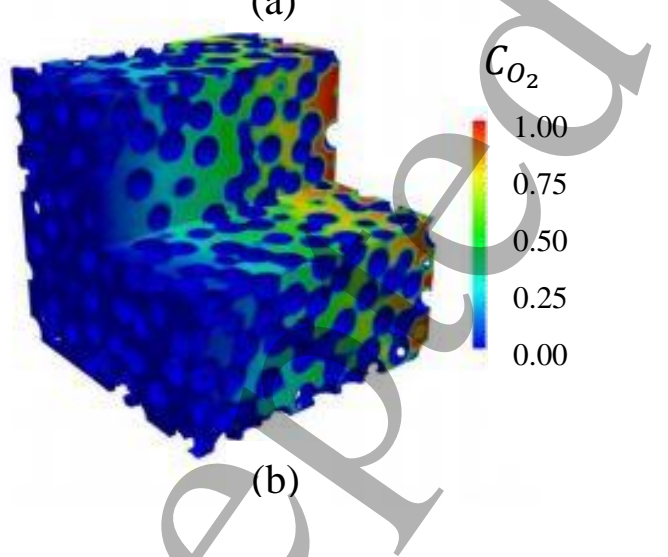

Figure 12: (a) liquid water distribution (with liquid water shown in blue and the gas phase in white) and (b) oxygen concentration distribution within the computational domain having a porosity value of 0.61 and a saturation level of 0.2. (reproduced from [223])

Pore-network models (PNMs) as a micro-scale model have been adopted to investigate liquid water transportinside the GDM $[230,231]$ and the impacts of mixed wettability on liquid water and reactant 
gas transport through the GDL of PEMFCs [232]. Ma et al. [233] investigated the limiting effect of strongly hydrophobic sub-micron pores on water transport in the MPL. As observed in experiments, simulation results indicate the structural preferential paths in an MPL are likely to be responsible for significant liquid water transport from the CL to GDL. Nano-tomography and pore-scale modeling were used to study the conflicting impacts caused by the existence of hydrophobic micro-porous layer [234]. The hydrophobic MPL in PEMFCs improves water management but reduces oxygen transport. The effect of compression on the distribution of liquid water and gaseous oxygen in the GDL was investigated by the pore networks with detailed information from tomography images of compressed GDLs [235]. Recently, a PNM has been developed to simulate the liquid water formation by vapor condensation in the GDL of a cathode when considering spatial temperature variations [236]. A newly developed PNM, referred to as the Mixed Injection Pore Network Model, is able to simulate both the liquid injection and the pore filling by liquid water due to condensation [237]. Some studies have used PNMs to explore the presence of two-phase flow in the CL [238-240]. For example, Hannach et al. [238] developed a pore network consisting of spheres and cylinders representative of pore spaces to study multi-phase transport phenomenon inside hydrophilic and hydrophobic catalyst layer of PEMFCs. It was observed that a change from hydrophobic to hydrophilic has a significant impact on the results of breakthrough point density and water saturation in the CL. The coupled transport and reaction processes in the cathode CL of PEMFC is investigated, and it is found that the cell voltage increases at the high current density with the CL thickness [241]. The PNM is useful in simulating the two-phase flow in porous electrodes. However, its inherent way of representing the porous structure using a simplified pore space geometry means that it has its own limitation by not able to capture the realistic microstructure.

The LBM is capable of simulating both single-phase [242,243] and multi-phase [179,244-253] flows in an arbitrary pore space geometry and topology rather than using a simplification of the pore space geometry. Therefore, LBM has been used to simulate realistic microstructures. A 3D LBM was developed to simulate the single-phase flow in cathode electrode of a PEMFC when considering the electrochemical reaction in the CL [180]. Simulation results demonstrate that normally oriented carbon fibres cause larger current variations on the CL. Fluid flow and mass transport in the gas channel and the GDL of a PEMFC were simulated by coupling FVM and LBM schemes [219, 220], which can not only capture the pore-scale information of fluid flow and species transport, but also save computational resource. The transport properties of carbon-paper and carbon cloth GDLs were further investigated using LBM [256]. The effective transport properties were calculated with the LBM in compressed GDL [257]. It is found that the results agree well with a trend based on the Kozeny-Carman equation. Recently, the GDL compression effects on the performance of a PEMFC 
cathode was presented by a LBM model [258]. It is found that there is an optimum compression factor of 0.85 for which the highest average current density is achieved. Moreover, LBM model was used to study the liquid water distribution in electrode. Mukherjee et al. [239] presented a LBM study of the influence of the pore structure and surface wettability on liquid water transport and interfacial dynamics in the PEMFC CL and GDL structure. The effects of the wettability and the anisotropic characteristics on relative permeabilities of the GDL were investigated by using the free energy multiphase LBM [259]. Moreover, the capillary pressures in a carbon paper GDL of PEMFCs were investigated and the results from LBM simulations are in good agreement with those obtained by experiment [260]. Kim et al. [67] investigated the liquid water transport in the MPL and GDL of PEMFC electrodes. The liquid water content can be reduced in the GDL by employing a thicker MPL and more hydrophobic surfaces, as shown in Figure 13. Jeon and Kim [179] revealed that the compression ratio significantly affects the water transport in the GDL. Most recently, the transport of liquid water inside the GDL was revealed by using LBM [261,262]. The overall LBM predictions of water evolution within the GDL agree well with the in-situ flow visualization from X-ray CT. Zhang et al. [68] proposed a 3D LBM to simulate liquid water transport in GDL of PEMFC with electrochemical reaction on the catalyst layer and oxygen diffusion in GDL taken into account in transient state. The modeling study showed that the existence of MPL embedded in GDL improved the mass transport and enhanced the performance of the PEMFCs, as shown in Figure 14.
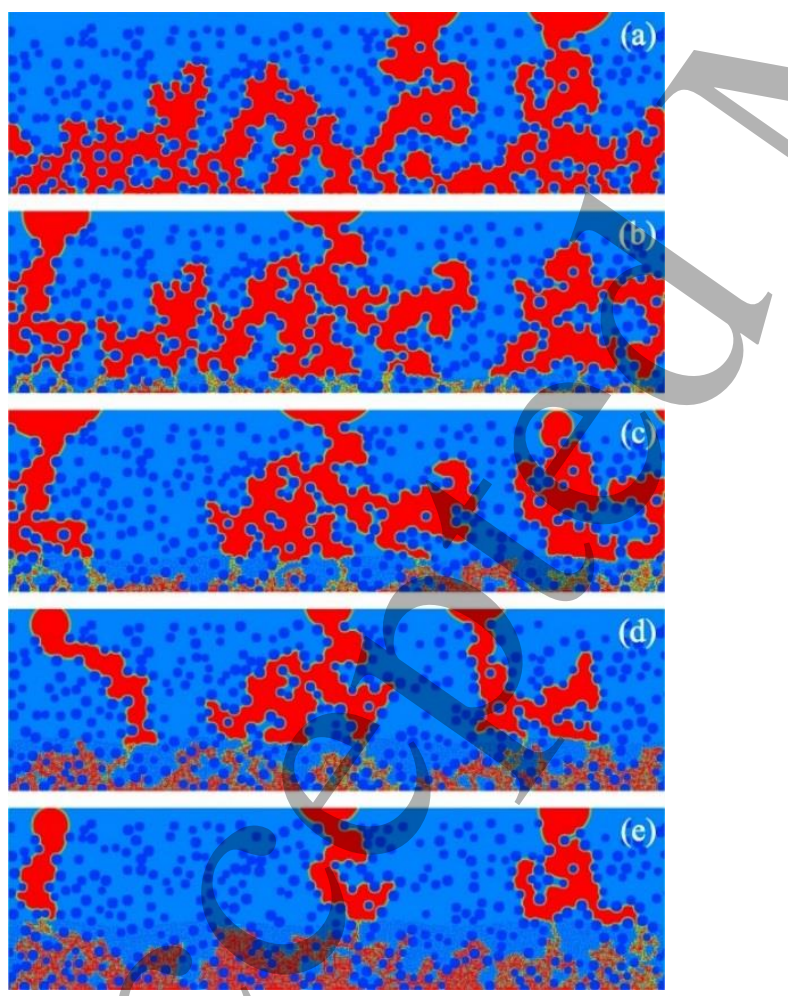

Figure 13: Steady-state distribution of liquid water clusters in GDL[67]: (a) without an MPL (LMPL = $0 \mu \mathrm{m}$ ), (b) $\mathrm{L}_{M P L}=25 \mu \mathrm{m}$, (c) $\mathrm{L}_{M P L}=25 \mu \mathrm{m}$, (d) $\mathrm{L}_{M P L}=75 \mu \mathrm{m}$, (e) $\mathrm{L}_{M P L}=100 \mu \mathrm{m}$. 


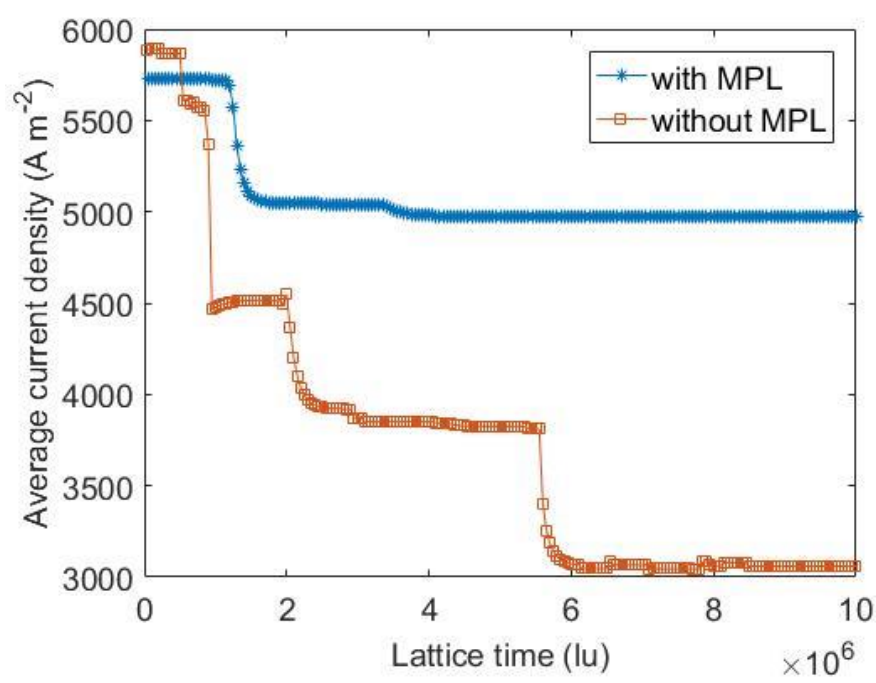

Figure 14: Time evolution of average current density on catalyst layer for different artificial cathode electrodes (reproduced from ref [68]).

In addition to GDL, LBM is used to study cathode CL/which is another critical component of PEMFCs [181,263-268]. Shin et al.[268] studied the mass transport properties and catalyst utilization of the CL on the performance of PEMFC using a 3D LBM model based on the quasi-random nanostructural model. Based on microstructures reconstructed by the sphere-based simulated annealing method, the LBM was used to evaluate the effects of the CL structural parameters, water saturation and Knudsen diffusion on the effective oxygen diffusivity in the CL [265]. Molaeimanesh and Akbari [267] proposed a 2D LBM agglomerate model to simulate electrochemical reaction in the catalyst layer. Then, they developed a 3D LBM agglomerate model to study the species distributions, electrical potential distribution in the electrolyte film and current density distribution at the interface of the CL [266]. The agglomerates of carbon black particles are considered as ellipsoids which can have different level of stretching. Oxygen and water vapor mole fraction variation is unsmooth and disturbed inside the CL, and the manner becomes more severe by increasing of ellipsoid stretching. Most recently, Chen et al. [181] conducted a pore-scale simulation based on LBM to study oxygen transport in the CL including carbon particle, ionomer, $\mathrm{Pt}$ particles and primary pores inside the carbon particle. It was suggested that local transport characteristics should be considered when developing 1D agglomeration model of CL.

So far, LBM has been very successful in simulating the gas and water transport in the CL and GDL with electrochemical reactions. To be able to simulate the CL more accurately, the process of liquid water formation from water vapor should be included into further LBM simulations. In the pore scale model, it is quite difficult to simulate the phase change phenomenon occurs in the CL; most of 
research assumes pure gas transport in the $\mathrm{CL}$ with chemical reaction and the liquid water-gas twophase flow in the GDL. The phase change from gas to liquid is neglected and its impact on the performance of PEMFC cannot be evaluated. Moreover, the marked difference in the pore size between the GDL $(\sim \mu \mathrm{m})$ and the $\mathrm{CL}(\sim \mathrm{nm})$ also brings challenges to the pore scale simulation. It is difficult to include both GDL and CL in one simulation as it requires very fine grid across both GDL and $\mathrm{CL}$, making the computation too expensive. One could argue that the predicted performance based on the simulations of the CL microstructure alone cannot truly represent the performance of the whole PEMFC electrode. Therefore no validation of the pore-scale modeling based on the CL structure has been made to compare the polarization curves from simulation and experimental measurement. Nonetheless, validation of the model based on the comparison of the effective diffusivity, effective conductivity [222] and water distribution in the GDL [261,262] have been attempted.

\subsection{Redox flow batteries (RFBs)}

The application of numerical modeling can provide a fundamental understanding of the coupled fluid flow, ion transport, electron conduction, and electrochemical reaction that occur in RFB electrodes [25]. The numerical models that have been developed for RFBs can be classified into macroscopic continuum models and microstructure-based pore-scale-models. In macroscopic continuum models [26,27,269-271], volumetric averaging conservation equations, such as mass, momentum, charge and energy, are solved in each elementary volume and the porous electrode is assumed as homogeneous continuum with isotropic or anisotropic transport properties. Most continuum models have been used to simulate cell performance [238-243]. The continuum models have also been made to investigate the effect of the electrode properties on the cell performance. For example, a recent continuum model developed for a hydrogen-bromine RFB considered the effect of the porous electrode by manipulating different porosity and surface area values, as well as the overall architecture of the cell [272]. It was found that the smaller fibres increase the surface area of the electrode to allow for more reactions to take place simultaneously. The formation of oxygen and hydrogen bubbles due to the side reactions and their effects on the performance of a vanadium RFB were also studied by a multi-phase mixture model [27,269]. Although useful for predicting the cell performance, the continuum models at the cell scale cannot be used to evaluate the precise effect of the electrode microstructure on the RFB performance, especially as there is no detailed information on how the wetting surface area available for electrochemical reactions affects the performance of RFBs. Alternatively, the pore-scale models provide an/efficient way for in-depth understanding of transport phenomena in different porous electrodes. Kok et al.[273] presented a study to determine the mass transfer coefficient in three 
commercial fibrous media for RFBs using LBM and direct numerical simulations on 3D tomograms. PNM approaches have also been used to investigate different transport properties of four carbon felt electrodes for vanadium RFBs [274]. The single-phase diffusion and permeability were found to decrease linearly with an increase in electrode thickness. Most recently, liquid-gas two-phase flow without reaction in the fibrous electrodes of vanadium RFBs was simulated using the LBM [69] to study the effects of porosity, fibre diameter, wettability of the fibre surface, saturation of the porous electrode on gas bubble migration, and reduction of reactive surface area due to coverage of bubbles on solid surface. The gas coverage ratio from the pore-scale model was lower than that adopted in the continuum model in the literature [269].

In order to model cell performance by incorporating the coupled transport and reaction processes, Qiu et al. [30,101] adopted the LBM to model electrolyte flow through a porous electrode structure obtained using X-ray CT, while FVM was used to solve the coupled species and charge transport and predict the electrochemical performance of a vanadium RFB. The simulation results clearly showed that the cell voltage increases with increasing electrolyte flow rate due to the decrease of concentration gradients of vanadium species in the porous electrode, and it tends to a marginal value once the concentration field approaches uniformity. The air bubbles produced from side reactions will occupy the pore space used for liquid electrolyte transport and affect the performance. Zhang et al. [31] developed a 3D LBM framework to simulate the transport of electrolyte and species, coupled with charge transfer in RFB electrode microstructures. This LBM framework enables the study of the effect of wetting area in carbon paper electrode on the performance of a vanadium RFB electrode. It was found that the electrochemical performance of positive half-cell is reduced with air bubbles trapped inside the electrode. In previous studies, the gas bubble distribution is considered as a steady state with the electrochemical reaction occurs at the interface between the electrolyte and solid fibres. Actually, the process that gas bubbles generate from the side reactions and transport in the porous electrode is a dynamic process. So, the assumption of the steady state gas bubble distribution cannot describe the real physical phenomenon in the RFB. The dynamic evolution of gas bubbles with electrochemical reaction is currently not able to be captured by pore-scale modelling.

The 3D pore-scale LBM model provides a powerful tool to study the real electrode microstructures, and to investigate their effects on cell performance. In a more recent study, Zhang et al. [182] have made efforts to validate their 3D pore-scale LBM model, by direct comparisons between modeled electrochemical performance and experimental measurement based on the same electrode materials applied in simulations and experiments. Excellent agreement was achieved between the simulated and experimentally measured performance, indicating the validity of the model for predicting RFB 
performance. Future work should focus on the optimization of electrode microstructure to improve the performance of RFB electrodes.

\subsection{Lithium-ion batteries (LIBs)}

The complex microstructure of the LIB electrodes has a significant effect on their performance. Numerous numerical methods have been proposed to improve the understanding of the transport phenomenon in porous electrodes as well as the relationship between the macroscopic battery performance and the microscopic transport. Initially, Newman et al. [275-277] developed a meanfield pseudo two-dimensional (P2D) model which used homogenized spatial distribution of electrode material and electrode porosity to reflect the microscopic effect. In subsequent years, the P2D model has been extended by many researchers [278-282]. Although the P2D model helped improve the battery design in some aspect, the impact of the electrode microstructure cannot be explicitly investigated in such a model. The model assumes certain bulk behavior and homogeneity which is not present in real structures. Nevertheless the model has been widely adopted and has considerable value.

In order to consider the microstructure of the battery electrode on the LIB performance, there is a growing interest to develop 2D [144] and 3D microstructure-resolved LIB models [80,161,283,284]. The FEM [111,143,285] and FVM methods [171,172,286,287] have often been utilized to solve the charge and species transport governing equation for LIB modeling due to readily available commercial software packages, such as COMSOL, StarCD++ and ANSYS. The 3D pore scale simulation of Galvanostatic discharge of $\mathrm{LiCoO}_{2}$ cathode based on X-ray Nano-CT images is shown in Figure 15 [46]. Reinholz et al. [142] adopted FEM simulations to reveal important challenges in the combined experimental and computational investigation of the link between processing, composition, microstructure, and the electrical conductivity of pressed-powder $\mathrm{Li} / \mathrm{FeS}_{2}$ thermal battery cathodes. Moreover, recent studies demonstrate that the inclusion of the carbon-binder domain (CBD) content changes the morphology and the ionic and electronic transport parameters dramatically, and thus cannot be neglected [77,104,106,161,288,289] in the electrode microstructure. The existence of CBD ensures sufficient electron conduction and mechanical rigidity but they also reduce the available reaction sites by reducing active material-electrolyte contact area. In addition, the pore phase transport resistance decreased as the CBD phase content is increased [77]. Trembacki et al. [78] presented a novel method to place a composite binder phase throughout the mesostructure, and compare effective thermal, electrical, and ionic conductivities across several binder placement approaches. Dependence of effective transport properties as a function of PVDF/carbon black loading 
and porosity would provide more information to microstructure models that may wish to study the long-range effects of varying such parameters. In order to evaluate CBD content and morphology on the electrochemical performance, the macroscale model based on the Newman et al. [275-277] was developed [76,77,104,106,161,288,289], which cannot explicitly connect the CBD distribution inside electrode and the electrochemical performance. So, it is suggested that future research may focus on this direction.

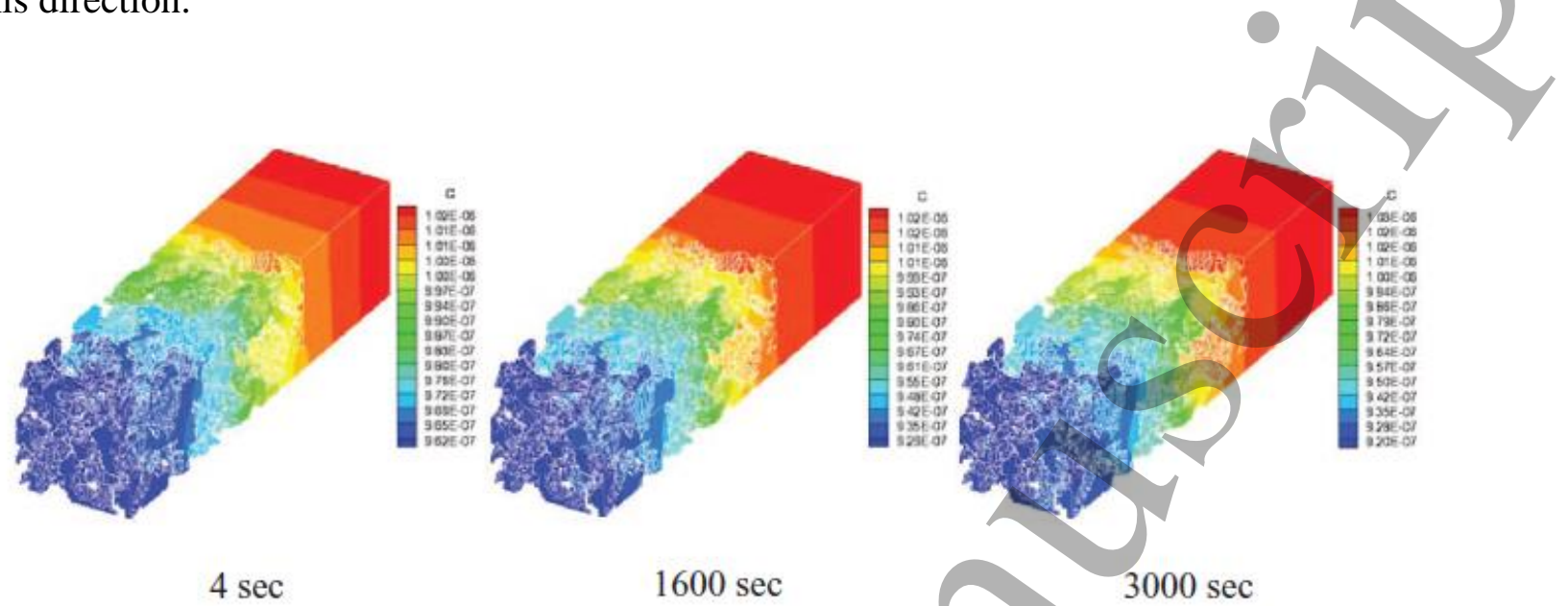

(a) concentration (unit: $\mathrm{mol} / \mathrm{mm}^{3}$ )

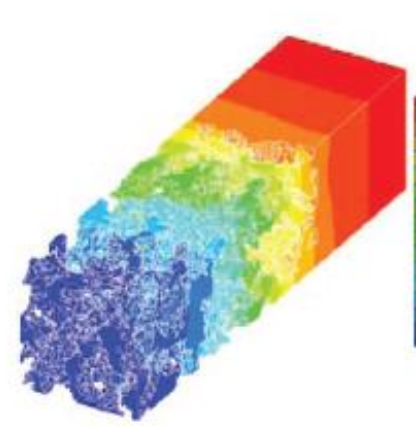

$4 \mathrm{~s}$ sec

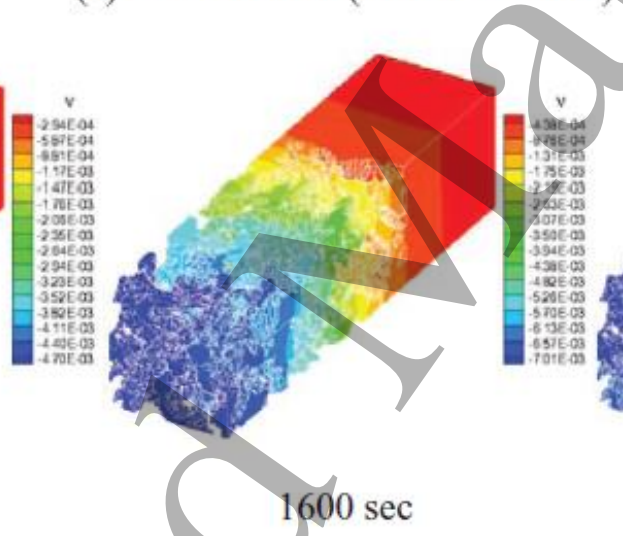

(b) voltage (unit: V)

Figure 15: Distribution of lithium ion concentration and voltage of electrolyte inside the electrode microstructure during $1 \mathrm{C}$ discharge process (reproduced from ref [46]).

The interaction behavior of the active material in a set of nickel-manganese-cobalt (NMC) cathodes was analyzed by microstructural characterization and computational modeling [290]. The result indicated that the particle size reduction from calendering and ball milling may improve rate capability. Electrochemical tests indicate that, during delithiation of graphite electrodes, the effective transport plays the most important role in cell performance [291]. Yan et al. [292] conducted a FVM study to simulate heat generation of a semi lithium ion battery during isothermal galvanostatic discharge processes. The microstructure of the LIB cathode electrode was reconstructed based on Xray nano computed technology. Simulation work demonstrated that inhomogeneous electrode 
microstructure leads to complicated and non-uniform spatial distribution of heat generation rates in the electrode during discharge processes. The heat generation calculated from 3D model based on the realistic microstructure is higher than that from porous-electrode theory model at large discharge rates. Mai et al. [34] employed a microstructure reconstruction algorithm to virtually build electrode microstructures and the effects of various microstructural features on the performance was investigated. The study showed that the shape/size of particles could affect the cell voltage. The larger particles in a cathode with a wide range of particle size distribution are not properly utilized.

However, difficulties may occur when dealing with the large complex porous electrode for FEM and FEM, and the computational time required for the calculation is enormous. Most recently, the LBM is also utilized for LIB modeling[183,184,293-296], owing to its ability to model complex transport processes with stochastic electrode geometries. He et al. [295] predicted the effective electrical and species transport properties of a LIB graphite anode via LBM. In the electrode through-plane direction, the effective electric and species transport properties decrease if the ellipsoidal particles are thinner and flatter. In addition, the simulation work indicated that the normal distribution of particle size increases the electronic charge conductivity in the through-plane direction and decreases in the inplane direction. Jiang et al. $[183,184]$ studied the relationship between the electrode microstructure and battery performance by developing a 2D LBM considering the ion and electron transport within an randomly reconstructed electrode geometry. The simulation work showed that a higher depth of discharge could be induced by a decrease in the porosity of the anode and an increase in the porosity of the cathode. It suggested that the particles of electrodes should be designed such that they are monodispersed and uniformly distributed to overcome the performance decline of LIBs. Lee $e t$ al. [293] evaluated the effect of the compression ratio of a porous electrode on wettability with variations of porosity and particle shape. The results showed that the effect of the compression ratio on wettability is significant. The area of liquid electrolyte (2D) in the compressed electrodes is reduced compared to uncompressed electrodes and the wetting capability is decreased with the increase of the compression ratio. Most recently, Mohammadian and Zhang [296] explored the effects of embedded microchannels inside the electrodes on electrolyte transport. It was revealed that the use of microchannels inside the electrodes significantly improves the wettability of the electrodes and could prevent the cell from thermal runaway occurrence.

Mechanics haye a critical influence on the performance and the lifetime of LIBs. LIBs suffer from chemo-mechanical degradation [297-299], which contributes significantly to capacity fade and impedance increase of a battery over charge/discharge cycles. So, numerical methods are developed to model the coupled chemo-mechanical behavior of LIBs $[33,79,107,143,299-307]$ by using FEM. 
For example, Roberts et al. [143] presented a framework for coupled simulations of electrode mechanics including swelling, deformation, and stress generation driven by lithium intercalation, as shown in Figure 16. It was demonstrated that for particles that swell isotropically, the largest stresses are located at the particle-to-particle contacts, due to neighboring particles constraining the swelling of a particle. Wu et al. [33,107] studied the stress generation caused by phase transitions and $\mathrm{Li}$ intercalation of NMC based half cell with realistic 3D microstructures, and it was shown that the stresses near the concave and convex regions are the highest. $\mathrm{Wu}$ and $\mathrm{Lu}$ [308] developed a multiscale mechanical-electrochemical model which enables fully coupled mechanics and electrochemistry at both particle and electrode levels. The stress arising from particle interaction simulated in particle level is incorporated in a continuum model. It has an important finding based on the simulation work that an electrochemically inactive region in an electrode causes stress built-up. A multiphysics microstructure-resolved model for LIB cells using alternative chemistries such as a Si anode was developed by Wang et al. [133]. The model was validated with experimental results, in which the electrochemical reactions, Li transport in electrolyte and electrodes, dimensional changes and stresses, property evolution with the structure, and the coupling relationships were considered. In-operando experiments coupled with stress models by Tariq et al. [79] for silicon based cells showed that peak stresses at anode-current collector interfaces cause failure at this surface and the progression speed is proportional to the peak stress experienced. Kim et al. [32] coupled electrochemical-mechanical-thermal factors in a multiphysics FEM model to investigate thermal-and diffusion-induced stresses with the reconstructed porous microstructures of commercial $\mathrm{LiFePO}_{4}$ batteries. Increasing temperature results in larger thermal stresses and higher diffusion-induced stresses were observed at the connecting areas between particles. It was suggested that the higher stresses may result from higher concentration variations in the connecting area. The study on the mechanism of lithium dendrite growth and its effect on the electrode performance is probably the next research direction of pore-scale modeling.

The key outcome revealed from LIB modelling is the true effect of inhomogeneity of the electrode microstructures on performance when considering real or near real structures. These are often computationally expensive to run, with computationally cheaper solutions sought while maintaining accuracy. The sources of inhomogeneity in the electrode (stress, intercalation, thermal etc) are all sources for initiating degradation within the battery which subsequently limit lifetime. In contrast, the bulk models are exceptionally good at calculating mean expected performance of a battery with certain attributes (e.g. electrode particle sizes etc) and provide a starting ball-park of expected performance and are computationally easier than fully coupled 3D microstructure models. A 
combination of the two, or their integration with each other, as discussed here is often needed to provide a comprehensive view of LIB performance.

It is essential to validate the microstructure resolved modeling study for the LIB. Microstructureresolved modeling has been widely used in the simulation research on the LIB, but the validation of the model against experiment has not received significant attention. To our knowledge, only a few studies $[66,67,255]$ have compared their 3D microstructure-resolved simulation results with the experimental measurements for the half-cell or full-cell galvanostatic discharge performance.
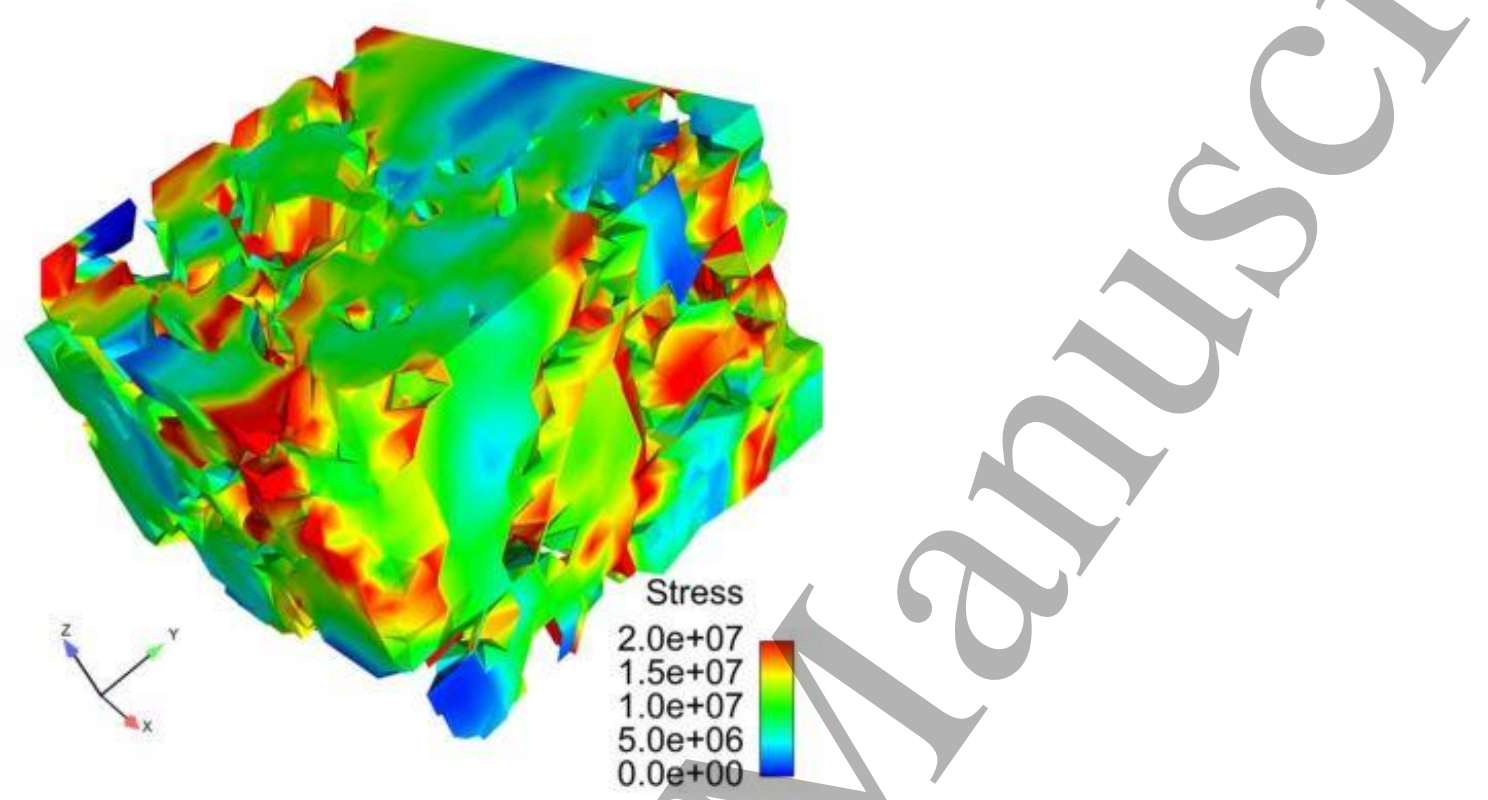

Figure 16: Von Mises stress in the solid electrode, in units of Pascals (reproduced from ref [143]).

\section{Conclusions and Prospects}

We have reviewed extensively the current development in the field of 3D microstructure resolved modelling and performance simulations for four popular electrochemical energy technologies including solid oxide fuel cells (SOFCs), proton exchange membrane fuel cells (PEMFCs), redox flow batteries (RFBs) and lithium ion batteries (LIBs). Particular attention has been given to three most widely used and proven methods including finite element method (FEM), finite volume method (FVM) and lattice Boltzmann method (LBM). Compared to continuum models, the advantage of the microstructure modelling lies in its ability of directly perform transport and performance simulations on 3D microstructures that may be synthetic (generated in computers), or real (obtained from FIBSEM or X-ray tomography), therefore allowing us to relate the microstructure to the electrochemical performance. Microstructure modelling is particularly useful when the electrode structures cannot be 
treated as homogenous media (which is the case for most of the electrodes in fuel cells and batteries), and when it is necessary to understand how a local defect or artefact affects the performance or lifetime.

Comparing different methods, FEM and FVM are established with a good number of commercial software and open-source platforms available to use, and thus have been widely applied in research. These existing platforms provide means to couple different chemical and physical phenomenon within the microstructure, including transport, electrochemical reactions, thermal and mechanical behaviours. FEM and FVM have been extensively applied in simulating electrodes in SOFCs and LIBs, for which the species transport within the porous space is validly treated as a single phase (e.g. gas phase for SOFCs and liquid phase for LIBs). The applications of FEM and FVM to simulating electrodes in PEMFCs and RFBs have been limited, mainly because of the challenges of simulating gas-liquid two-phase flow within the 3D microstructure, which is important as the existence of water in PEMFCs and gas bubbles in RFBs will make some part of the electrode inaccessible for reactions and affect the performance. LBM, on the other hand, is well suited to simulating multiphase flow and has therefore been used for studying electrodes in PEMFCs and RFBs. A relatively new method, the development and applications of LBM for microstructure modelling in fuel cells and batteries is the focus of current research efforts from individual groups who have made considerable progress in simulating transport and multiphase flow, coupled with electrochemical performance.

The trend in microstructure modelling is going from simulating a single physical/chemical process (such as heat transfer, transport, charge transfer, mechanical stress-strain behaviour) to coupled multiphysics. LIB modelling has reached quite a high level of maturity in terms of transportelectrochemical-thermal-mechanical coupling. In other technologies, the integration of transport with electrochemical reactions has been achieved, with thermal and mechanical effects often being neglected. Whilst coupled simulations provide the ability to correlate different performance indicators and better understand the structure-performance relation, it also imposes challenges. For FEM and FVM based simulations, it is important to have a good mesh to achieve reliable results. Indeed, having a solid and reliable meshing software is probably one of the most critical aspects in the whole workflow. Normally the most demanding mesh requirements are given by the field which shows stronger gradients (for example, Li solid diffusion in LIBs). However, such an accurate mesh is not necessary for other physics (e.g., heat flow, where temperature gradients are not so steep). It is still an open question if it would be better to use the finer mesh for all the physics or using different meshes (finer and coarser within the same domain, e.g., to solve for Li concentration and temperature within the same particle) for different physics: i.e. which approach is more computationally efficient? Particularly when simulating phenomena taking place at different length scales, it is typically the 
small-scale phenomenon that dictates the mesh requirements, so that a fine mesh is used to solve also for physics which experience smoother gradients, which inevitably increases the computational cost. Implementing efficient multiple-length scale modelling is still a challenge. For LBM based simulations, there have been a few recent developments in coupling transport with electrochemical reactions for batteries and fuel cells, achieved high efficiency and accuracy validated by experimental results. Coupling heat transfer in LBM is possible and has been done for other/applications. Nonetheless, such coupling has not been done for electrochemical energy devices.

Challenges still remain in 3D microstructure modelling to capture the necessary physical/chemical processes. Particularly when the microstructure consists of pores at the nanometer scale (which is often the case now with nanomaterials widely used in energy devices including fuel cells and batteries), Knudsen diffusion becomes important for SOFCs and PEMFCs where gas transports within the porous structure. However, methodologies to include Knudsen diffusion in the current transport simulation framework remain challenging. Moreover, for simulating gas transport, the Dusty-Gas Model (DGM) is not suited for 3D microstructure-resolved simulations as the DGM implicitly encodes the continuum approximation. So, including Knudsen diffusion (beyond the Bosanquet equation, which is an approximation) in FEM/FVM/LBM frameworks is still an open question. For PEMFCs, there is phase transition happening within the CL structure, where the generated vapour becomes liquid water. The CL porous structure may play a key role in this phase transition process. However, whether it would be possible to include the simulation of this phase transition in the 3D microstructure-resolved simulations is still a question.

Furthermore, the current research in 3D microstructure-resolved simulations is not well equipped with the capability of simulating the structural changes typically happening in SOFCs (where $\mathrm{Ni}$ particle coarsening is critical to performance degradation) and LIBs ( where during battery charge/discharge, electrode/materials particles may undergo swelling/shrinking, particle fracture, thermal expansion, phase separation, etc.). It is difficult to track domain deformation or interface movement in 3D and adapt the mesh and the boundary conditions to predict the different physical and chemical processes. It is still an open question how far a phase field approach can be used or if it is better using adaptative mesh refinement methods.

For simulating electrochemical performance, the Butler-Volmer equation has demonstrated applicability and is commonly used. However, it is recognized that Butler-Volmer (BV) equation has the following limitations. It assumes that charge transfer is rate determining, which is not always the case. It is a generalized and averaged equation for the overall reaction, and does not give information on the elementary chemistry and individual reaction steps (e.g. adsorption, surface migration, charge 
transfer, desorption etc) involved. The structure effects on these elementary reactions/steps are thus overlooked. Furthermore, it is a challenge to obtain a reliable reaction rates as a key input to the BV equation. The reaction rate is often taken from literature, which means the value does not necessarily correspond to the simulated system as it relies on the specific materials and the operation conditions etc.

It is clearly important to validate the $3 \mathrm{D}$ microstructure modelling. Although validation has been reported with SOFCs and RFBs, it has been scarce for PEMFCs and LIBs. An approach has been established by the authors in experimental validation of the 3D microstructure modelling, by combining with 3D tomography and experimental measurement. In this approach, the "real" microstructure obtained from 3D tomography (FIB-SEM or X-ray CT) is input to the model for simulating the physical and chemical processes. The simulated performance indicators are then compared against the experimentally measured values, to validate the model and provide sensors for improving the model. We recommend that research groups in $3 \mathrm{D}$ microstructure modelling collaborate closely with experimental groups, to obtain reliable reaction rate parameters and to validate and improve the model.

To date, 3D microstructure modelling studies have mostly been used to get averaged properties or global predictions, such as I-V curves or macro-scopic effective properties. To take full advantage of 3D microstructures, we need to find proper 3D microstructural descriptors and couple them with 3D simulations to assess the heterogeneous distribution of temperature, concentrations, current generation, etc. A seminal study is the approach that the authors developed based on particle statistics. The research community should build upon it and use 3D simulations to get truly 3D information (about hot spots, mal-distributions, etc.) that cannot be predicted by continuum models.

The 3D microstructure modelling provides a direct link between the intrinsic 3D microstructures and the transport and electrochemical performance. It therefore opens the door to bottom-up design of new electrode structures to improve device performance. This is fundamentally different from continuum modelling, in which the "design" is optimised by manipulating the averaged structural properties (such as porosity, tortúosity, mean pore size etc). Although averaged structural properties can be useful, they do not provide direct link to a specific structure and, in turn, to specific manufacturing approaches; indeed, there may be a number of structures with different local functions and giving different performance yet sharing the same averaged structural properties. This poses serious limitations for the continuum modelling approach to inform the design about the effect of local phenomena, such as degradation mechanisms. 3D microstructure models, on the other hand, can work with a specific structure and examine the effect of any local morphology, thus pointing towards 
an optimisation strategy which takes into account both the macroscopic electrode response and the microscopic localised effects. Novel electrode microstructures can be created or generated in computers, with their transport and electrochemical performance investigated using 3D microstructure modelling. We believe that 3D microstructure modelling provides a powerful tool for designing next-generation fuel cells and battery electrodes, which can be realized with novel manufacturing techniques.

\section{Acknowledgement}

DZ and QC are grateful for the financial support from the UK Engineering and Physical Science Research Council (EPSRC) (grant number EP/ M027066/1, EP/R512904). The authors wish to acknowledge the unknown reviewers whose detailed and insightful comments significantly improved the quality and clarity of the paper.

\section{References}

[1] R.M. Ormerod, Solid oxide fuel cells, Chem.Soc.Rev. 32(2003) 17-28. doi:10.1039/B105764M.

[2] S.J. Cooper, N.P. Brandon, An Introduction to Solid Oxide Fuel Cell Materials, Technology and Applications, in: N.P. Brandon, E. Ruiz-Trejo, P. Boldrin (Eds.), Solid Oxide Fuel Cell Lifetime Reliab. Crit. Challenges Fuel Cells, Elsevier Academic Press, London, 2017: pp. 118. doi:10.1016/B978-0-08-101102-7.00001-5.

[3] M.M. Menchhh, Fuel Cell Enginesss Fuel Cell Engines, John Wiley \& Sons, 2008. http://www.wiley.com/go/permissions.

[4] A. Jayakumar, S.P. Sethu, M. Ramos, J. Robertson, A. Al-Jumaily, A technical review on gas diffusion, mechanism and medium of PEM fuel cell, Ionics (Kiel). 21 (2015) 1-18. doi:10.1007/s 11581-014-1322-x.

[5] Z. Yang, J. Zhang, M.C.W. Kintner-Meyer, X. Lu, D. Choi, J.P. Lemmon, J. Liu, Electrochemical Energy Storage for Green Grid, Chem. Rev. 111 (2011) 3577-3613. doi:10.1021/cr100290v.

[6] L. Li, X. Wei, B. Li, Z. Yang, Q. Luo, W. Wang, Recent Progress in Redox Flow Battery Research and Development, Adv. Funct. Mater. 23 (2012) 970-986. doi:10.1002/adfm.201200694.

[7] C. Ding, H. Zhang, X. Li, T. Liu, F. Xing, Vanadium flow battery for energy storage: Prospects and challenges, J. Phys. Chem. Lett. 4 (2013) 1281-1294. doi:10.1021/jz4001032. 
[8] F. Tariq, J. Rubio-Garcia, V. Yufit, A. Bertei, B.K. Chakrabarti, A. Kucernak, N. Brandon, Uncovering the mechanisms of electrolyte permeation in porous electrodes for redox flow batteries through real time in situ 3D imaging, Sustain. Energy Fuels. 2 (2018) 2068-2080. doi:10.1039/c8se00174j.

[9] B. Scrosati, J. Garche, Lithium batteries: Status, prospects and future, J. Power Sources. 195 (2010) 2419-2430. doi:10.1016/j.jpowsour.2009.11.048.

[10] B.C.H. Steele, Survey of materials selection for ceramic fuel cells: II. Cathodes and anodes, Solid State Ionics. 86-88 (1996) 1223-1234. doi:10.1016/0167-2738(96)00291-3.

[11] S. Litster, G. McLean, PEM fuel cell electrodes, J. Power Sources. 130 (2004) 61-76. doi:org/10.1016/j.jpowsour.2003.12.055.

[12] K.J. Kim, M.-S. Park, Y.-J. Kim, J.H. Kim, S.X. Dou, M. Skyllas-Kazacos, A technology review of electrodes and reaction mechanisms in vanadium redox flow batteries, J. Mater. Chem. A. 3 (2015) 16913-16933. doi:10.1039/C5TA02613J.

[13] M. Kishimoto, M. Lomberg, E. Ruiz-Trejo, N.P. Brandon, Enhanced triple-phase boundary density in infiltrated electrodes for solid oxide fuel cells demonstrated by high-resolution tomography, J. Power Sources. 266 (2014) 291-295. doi:10.1016/j.jpowsour.2014.05.038.

[14] S. Amitai, A. Bertei, R. Blumenfeld, Theory-based design of sintered granular composites triples three-phase boundary in fuel cells, Phys. Rev. E. 96 (2017) 052903. doi:10.1103/PhysRevE.96.052903,

[15] X. Lu, T. Li, A. Bertei, J.I.S. Cho, T.M.M. Heenan, M.F. Rabuni, K. Li, D.J.L. Brett, P.R. Shearing, The application of hierarchical structures in energy devices: New insights into the design of solid oxide fuel cells with enhanced mass transport, Energy Environ. Sci. 11 (2018) 2390-2403. doi:10.1039/c8ee01064a.

[16] T. Tanuma, M. Kawamoto, S. Kinoshita, Effect of Properties of Hydrophilic Microporous Layer (MPL) on PEFC Performance, J. Electrochem. Soc. 164 (2017) F499-F503. doi:10.1149/2.0371706jes.

[17] W. Zheng, S.H. Kim, The Effects of Catalyst Layer Microstructure and Water Saturation on the Effective Diffusivity in PEMFC, J. Electrochem. Soc. 165 (2018) F468-F478. doi:10.1149/2.0711807jes.

[18] X.L. Zhou, T.S. Zhao, Y.K. Zeng, L. An, L. Wei, A highly permeable and enhanced surface area carbon-cloth electrode for vanadium redox flow batteries, J. Power Sources. 329 (2016) 247-254. doi:10.1016/j.jpowsour.2016.08.085.

[19] V.Etacheri, R. Marom, R. Elazari, G. Salitra, D. Aurbach, Challenges in the development of advanced Li-ion batteries: a review, Energy Environ. Sci. 4 (2011) 3243-3262.

doi:10.1039/C1EE01598B. 
[20] A.N. Mistry, P.P. Mukherjee, Probing spatial coupling of resistive modes in porous intercalation electrodes through impedance spectroscopy, Phys. Chem. Chem. Phys. 21 (2019) 3805-3813. doi:10.1039/c8cp05109g.

[21] A. Bertei, F. Tariq, V. Yufit, E. Ruiz-Trejo, N.P. Brandon, Guidelines for the Rational Design and Engineering of 3D Manufactured Solid Oxide Fuel Cell Composite Electrodes, in: J. Electrochem. Soc., 2016: pp. F89-F98. doi:10.1149/2.0501702jes.

[22] W.G. Bessler, S. Gewies, M. Vogler, A new framework for physically based modeling of solid oxide fuel cells, Electrochim. Acta. 53 (2007) 1782-1800. doi:10.1016/j.electacta.2007.08.030.

[23] P.C. Sui, S. Kumar, N. Djilali, Advanced computational tools for PEM fuel cell design, J. Power Sources. 180 (2008) 410-422. doi:10.1016/j.jpowsour.2008.02.007.

[24] W. Lai, F. Ciucci, Mathematical modeling of porous battery electrodes-Revisit of Newman's model, Electrochim. Acta. 56 (2011) 4369-4377. doi:10.1016/j.electacta.2011.01.012.

[25] Q. Xu, T.S. Zhao, Fundamental models for flow batteries, Prog. Energy Combust. Sci. 49 (2015) 40-58. doi:10.1016/j.pecs.2015.02.001.

[26] D. You, H. Zhang, J. Chen, A simple model for the vanadium redox battery, Electrochim. Acta. 54 (2009) 6827-6836. doi:10.1016/j.electacta.2009.06.086.

[27] H. Al-Fetlawi, A.A. Shah, F.C. Walsh, Modelling the effects of oxygen evolution in the allvanadium redox flow battery, Electrochim. Acta. 55 (2010) 3192-3205. doi:10.1016/j.electacta.2009.12.085.

[28] Lei Xing, Qiong Cai, Xiaoteng Liu, Chunbo Liu, K. Scott, Yongsheng Yan, Anode partial flooding modelling of proton exchange membrane fuel cells: optimisation of electrode properties and channel geometries, Chem. Eng. Sci. 146 (2016) 88-103. doi:org/10.1016/j.ces.2016.02.029.

[29] Q. Cai, C.S. Adjiman, N.P. Brandon, Modelling the 3D microstructure and performance of solid oxide fuel cell electrodes: Computational parameters, Electrochim. Acta. 56 (2011) 5804-5814. doi:10.1016/j.electacta.2011.04.065.

[30] G. Qiu, E.C. Kumbur, Y. Sun, A.S. Joshi, K.W. Knehr, C.R. Dennison, 3-D pore-scale resolved model for coupled species/charge/fluid transport in a vanadium redox flow battery, Electrochim. Acta. 64 (2012) 46-64. doi:10.1016/j.electacta.2011.12.065.

[31] D. Zhang, Q. Cai, O.O. Taiwo, V. Yufit, N.P. Brandon, S. Gu, The effect of wetting area in carbon paper electrode on the performance of vanadium redox flow batteries: A threedimensional lattice Boltzmann study, Electrochim. Acta. 283 (2018) 1806-1819. doi:10.1016/j.electacta.2018.07.027. 
[32] S. Kim, J. Wee, K. Peters, H.Y.S. Huang, Multiphysics coupling in lithium-ion batteries with reconstructed porous microstructures, J. Phys. Chem. C. 122 (2018) 5280-5290. doi:10.1021/acs.jpcc.7b12388.

[33] L. Wu, Y. Wen, J. Zhang, Three-Dimensional Finite Element Study on Li Diffusion Induced Stress in FIB-SEM Reconstructed LiCoO2Half Cell, Electrochim. Acta. 222 (2016) 814820. doi:10.1016/j.electacta.2016.11.042.

[34] W. Mai, M. Yang, S. Soghrati, A particle-resolved 3D finite element model to study the effect of cathode microstructure on the behavior of lithium ion batteries, Electrochim. Acta. 294 (2019) 192-209. doi:10.1016/j.electacta.2018.10.072.

[35] G.M. Goldin, A.M. Colclasure, A.H. Wiedemann, R.J. Kee, Three-dimensional particleresolved models of Li-ion batteries to assist the evaluation of empirical parameters in onedimensional models, Electrochim. Acta. 64 (2012) 118-129. doi:10.1016/j.electacta.2011.12.119.

[36] T.D. Le, D. Lasseux, X.P. Nguyen, G. Vignoles, N. Mano, A. Kuhn, Multi-scale modeling of diffusion and electrochemical reactions in porous micro-electrodes, Chem. Eng. Sci. 173 (2017) 153-167. doi:10.1016/j.ces.2017.07.039.

[37] A.H. Wiedemann, G.M. Goldin, S.A. Barnett, H. Zhu, R.J. Kee, Effects of three-dimensional cathode microstructure on the performance of lithium-ion battery cathodes, Electrochim. Acta. 88 (2013) 580-588. doi:10.1016/j.electacta.2012.10.104.

[38] A.G. Kashkooli, A. Amirfazli, S. Farhad, D.U. Lee, S. Felicelli, H.W. Park, K. Feng, V. De Andrade, Z. Chen, Representative volume element model of lithium-ion battery electrodes based on X-ray nano-tomography, J. Appl. Electrochem. 47 (2017) 281-293. doi:10.1007/s 10800-016-1037-y.

[39] G. Ceder, Opportunities and challenges for first-principles materials design and applications to Li battery materials, MRS Bull. 35 (2010) 693-701.

[40] R.R. Maphanga, P.E. Ngoepe, Computational Modelling as a Value Add in Energy Storage Materials, in: Nanomater. Adv. Batter. Supercapacitors, Springer, 2016: pp. 481-513.

[41] M.S. Islam, C.A.J. Fisher, Lithium and sodium battery cathode materials: computational insights into yoltage, diffúsion and nanostructural properties, Chem. Soc. Rev. 43 (2014) 185-204.

[42] A. Jain, Y. Shin, K.A. Persson, Computational predictions of energy materials using density functional theory, Nat. Rev. Mater. 1 (2016) 15004.

[43] K. Rhazaoui, Q. Cai, M. Kishimoto, F. Tariq, M.R. Somalu, C.S. Adjiman, N.P. Brandon, Towards the 3D modelling of the effective conductivity of solid oxide fuel cell electrodes - 
Validation against experimental measurements and prediction of electrochemical performance, Electrochim. Acta. 168 (2015) 139-147. doi:10.1016/j.electacta.2015.04.005.

[44] C. Wieser, T. Prill, K. Schladitz, Multiscale simulation process and application to additives in porous composite battery electrodes, J. Power Sources. 277 (2015) 64-75. doi:10.1016/j.jpowsour.2014.11.090.

[45] A.G. Kashkooli, S. Farhad, D.U. Lee, K. Feng, S. Litster, S.K. Babu, L. Zhu, Z. Chen, Multiscale modeling of lithium-ion battery electrodes based on nano-scale X-ray computed tomography, J. Power Sources. 307 (2016) 496-509. doi:10.1016/j.jpowsour.2015.12.134.

[46] B. Yan, C. Lim, L. Yin, L. Zhu, Three Dimensional Simulation of Galvanostatic Discharge of LiCoO 2 Cathode Based on X-ray Nano-CT Images , J. Electrochem. Soc. 159 (2012) A1604-A1614. doi:10.1149/2.024210jes.

[47] M. Ebner, F. Geldmacher, F. Marone, M. Stampanoni, V. Wood, X - ray tomography of porous, transition metal oxide based lithium ion battery electrodes, Adv. Energy Mater. 3 (2013) 845-850.

[48] P.T. Moseley, Fuel Cell Systems Explained, Wiley, New York, 2002. doi:10.1016/s03787753(00)00571-1.

[49] N.M. Sammes, K. Galloway, M.F. Serincan, T. Suzuki, T. Yamaguchi, M. Awano, W. Colella, Solid oxide fuel cells, Handb. Clim. Chang. Mitig. 4 (2012) 1703-1727. doi:10.1007/978-1-4419-7991-9_44.

[50] N.Q. Minh, Solid oxide fuel cell technology - Features and applications, Solid State Ionics. 174 (2004) 271-277. doi:10.1016/j.ssi.2004.07.042.

[51] W.H. Kan, A.J. Samson, V. Thangadurai, Trends in electrode development for next generation solid oxide fuel cells, J. Mater. Chem. A. 4 (2016) 17913-17932.

[52] S.B. Adler, Factors Governing Oxygen Reduction in Solid Oxide Fuel Cell Cathodes $\dagger$, Chem. Rev. 104 (2004) 4791-4844. doi:10.1021/cr020724o.

[53] P. Costamagna, P. Costa, V. Antonucci, Micro-modelling of solid oxide fuel cell electrodes, Electrochim. Acta. 43 (1998) 375-394. doi:10.1016/S0013-4686(97)00063-7.

[54] F. Tariq, N.P. Brandon, G. Cui, M. Somalu, V. Yufit, M. Kishimoto, 3D imaging and quantification of interfaces in SOFC anodes, J. Eur. Ceram. Soc. 34 (2014) 3755-3761. doi:10.1016/j.jeurceramsoc.2014.05.003.

[55] P. Costamagna, P. Costa, E. Arato, Some more considerations on the optimization of cermet solid oxide fuel cell electrodes, Electrochim. Acta. 43 (1998) 967-972. doi:10.1016/S00134686(97)00262-4.

[56] A. Atkinson, Solid Oxide Fuel Cell Electrolytes-Factors Influencing Lifetime, in: N.P. Brandon, E. Ruiz-Trejo, P. Boldrin (Eds.), Solid Oxide Fuel Cell Lifetime Reliab. Crit. 
Challenges Fuel Cells, Elsevier Academic Press, London, 2017: pp. 19-35. doi:10.1016/B978-0-08-101102-7.00002-7.

[57] S.J. Cooper, A. Bertei, P.R. Shearing, J.A. Kilner, N.P. Brandon, TauFactor: An open-source application for calculating tortuosity factors from tomographic data, SoftwareX. 5 (2016) 203-210. doi:10.1016/j.softx.2016.09.002.

[58] L. Holzer, D. Wiedenmann, B. Münch, L. Keller, M. Prestat, P. Gasser, I. Robertson, B. Grobéty, The influence of constrictivity on the effective transport properties of porous layers in electrolysis and fuel cells, J. Mater. Sci. 48 (2013) 2934-2952. doi:10.1007/s10853-0126968-z.

[59] K. Rhazaoui, Q. Cai, C.S. Adjiman, N.P. Brandon, Towards the 3D modeling of the effective conductivity of solid oxide fuel cell electrodes - II: Computational parameters, Chem. Eng. Sci. 116 (2014) 781-792. doi:10.1016/j.ces.2014.05.045.

[60] B. Kenney, M. Valdmanis, C. Baker, J.G. Pharoah, K. Karan, Computation of TPB length, surface area and pore size from numerical reconstruction of composite solid oxide fuel cell electrodes, J. Power Sources. 189 (2009) 1051-1059. doi:10.1016/j.jpowsour.2008.12.145.

[61] H. Zhu, R.J. Kee, V.M. Janardhanan, O. Deutschmann, D.G. Goodwin, Modeling Elementary Heterogeneous Chemistry and Electrochemistry in Solid-Oxide Fuel Cells, J. Electrochem. Soc. 152 (2005) A2427. doi:10.1149/1.2116607.

[62] V. Yurkiv, A. Utz, A. Weber, E. Ivers-Tiffée, H.-R. Volpp, W.G. Bessler, Elementary kinetic modeling and experimental validation of electrochemical CO oxidation on $\mathrm{Ni} / \mathrm{YSZ}$ pattern anodes, Electrochim. Acta. 59 (2012) 573-580. doi:10.1016/j.electacta.2011.11.020.

[63] G.J. Nelson, K.N. Grew, J.R. Izzo, J.J. Lombardo, W.M. Harris, A. Faes, A. Hessler-Wyser, J. Van Herle, S. Wang, Y.S. Chu, A. V. Virkar, W.K.S. Chiu, Three-dimensional microstructural changes in the Ni-YSZ solid oxide fuel cell anode during operation, Acta Mater. 60 (2012) 3491-3500. doi:10.1016/j.actamat.2012.02.041.

[64] Z. Jiao, N. Shikazono, Simulation of Solid Oxide Fuel Cell Anode Microstructure Evolution Using Phase Field Method, J. Electrochem. Soc. 160 (2013) F709-F715. doi:10.1149/2.139306jes.

[65] D. Kennouche, Y.C.K. Chen-Wiegart, K.J. Yakal-Kremski, J. Wang, J.W. Gibbs, P.W. Voorhees, S.A. Barnett, Observing the microstructural evolution of Ni-Yttria-stabilized zirconia solid oxide fuel cell anodes, Acta Mater. 103 (2016) 204-210. doi:10.1016/j.actamat.2015.09.055.

[66] R.K. Shah, Introduction to Fuel Cells BT - Recent Trends in Fuel Cell Science and Technology, in: Polym. Electrolyte Membr. Fuel Cell, Anamaya Publisher, 2007: pp. 1-9. doi:10.1007/978-0-387-68815-2_1. 
[67] K.N. Kim, J.H. Kang, S.G. Lee, J.H. Nam, C.-J. Kim, Lattice Boltzmann simulation of liquid water transport in microporous and gas diffusion layers of polymer electrolyte membrane fuel cells, J. Power Sources. 278 (2015) 703-717. doi:org/10.1016/j.jpowsour.2014.12.044.

[68] D. Zhang, Q. Cai, S. Gu, Three-dimensional lattice-Boltzmann model for liquid water transport and oxygen diffusion in cathode of polymer electrolyte membrane fuel cell with electrochemical reaction, Electrochim. Acta. 262 (2018) 282-296. doi:10.1016/j.electacta.2017.12.189.

[69] L. Chen, Y.L. He, W.Q. Tao, P. Zelenay, R. Mukundan, Q. Kang, Pore-scale study of multiphase reactive transport in fibrous electrodes of vanadium redox flow batteries, Electrochim. Acta. 248 (2017) 425-439. doi:10.1016/j.electacta.2017.07.086.

[70] M.K. Song, S. Park, F.M. Alamgir, J. Cho, M. Liu, Nanostructured electrodes for lithium-ion and lithium-air batteries: The latest developments, challenges, and perspectives, Mater. Sci. Eng. R Reports. 72 (2011) 203-252. doi:10.1016/j.mser.2011.06.001.

[71] A.S. Aricò, P. Bruce, B. Scrosati, J.-M. Tarascon, W.A. van Schalkwijk, Nanostructured materials for advanced energy conversion and storage devices, Nat. Mater. 4 (2005) 366377. doi:10.1038/nmat1368.

[72] C. Huang, N.P. Young, J. Zhang, H.J. Snaith, P.S. Grant, A two layer electrode structure for improved Li Ion diffusion and volumetric capacity in Li Ion batteries, Nano Energy. 31 (2017) 377-385. doi:10.1016/j.nanoen.2016.11.043.

[73] S.P. Sheu, C.Y. Yao, J.M. Chen, Y.C. Chiou, Influence of the LiCoO2 particle size on the performance of lithium-ion batteries, J. Power Sources. 68 (1997) 533-535. doi:10.1016/S0378-7753(97)02623-2.

[74] M.F. Lagadec, R. Zahn, S. Müller, V. Wood, Topological and network analysis of lithium ion battery components: The importance of pore space connectivity for cell operation, Energy Environ. Sci. 11 (2018) 3194-3200. doi:10.1039/c8ee00875b.

[75] C. Huang, P.S. Grant, Coral-like directional porosity lithium ion battery cathodes by ice templating, J. Mater. Chem. A. 6 (2018) 14689-14699. doi:10.1039/c8ta05049j.

[76] A. Mistry, D. Juarez-Robles, M. Stein, K. Smith, P.P. Mukherjee, Analysis of long-range interaction in lithium-ion battery electrodes, J. Electrochem. Energy Convers. Storage. 13 (2016) 031006. doi:10.1115/1.4035198.

[77] A.N. Mistry, K. Smith, P.P. Mukherjee, Secondary-phase stochastics in lithium-ion battery electrodes, ACS Appl. Mater. Interfaces. 10 (2018) 6317-6326.doi:10.1021/acsami.7b17771.

[78] B.L. Trembacki, D.R. Noble, V.E. Brunini, M.E. Ferraro, S.A. Roberts, Mesoscale Effective Property Simulations Incorporating Conductive Binder, J. Electrochem. Soc. 164 (2017) E3613-E3626. doi:10.1149/2.0601711jes. 
[79] F. Tariq, V. Yufit, D.S. Eastwood, Y. Merla, M. Biton, B. Wu, Z. Chen, K. Freedman, G. Offer, E. Peled, P.D. Lee, D. Golodnitsky, N. Brandon, In-Operando X-ray Tomography Study of Lithiation Induced Delamination of Si Based Anodes for Lithium-Ion Batteries, ECS Electrochem. Lett. 3 (2014) A76-A78. doi:10.1149/2.0081407eel.

[80] A.N. Mistry, K. Smith, P.P. Mukherjee, Electrochemistry coupled mesoscale complexations in electrodes lead to thermo-electrochemical extremes, ACS Appl. Mater. Interfaces. 10 (2018) 28644-28655. doi:10.1021/acsami.8b08993.

[81] F. Tariq, R. Haswell, P.D. Lee, D.W. McComb, Characterization of hierarchical pore structures in ceramics using multiscale tomography, Acta Mater. 59 (2011) 2109 22120. doi:10.1016/j.actamat.2010.12.012.

[82] M.D. Uchic, L. Holzer, B.J. Inkson, E.L. Principe, P. Munroe, Three-dimensional microstructural characterization using focused ion beam tomography, Mrs Bull. 32 (2007) 408-416.

[83] F. Tariq, P.D. Lee, R. Haswell, D.W. McComb, The influence of nanoscale microstructural variations on the pellet scale flow properties of hierarchical porous catalytic structures using multiscale 3D imaging, Chem. Eng. Sci. 66 (2011) 5804-5812.

[84] P. Shearing, Y. Wu, S.J. Harris, N. Brandon, In situ X-ray spectroscopy and imaging of battery materials, Electrochem. Soc. Interface. 20 (2011) 43-47.

[85] D. Kanno, N. Shikazono, N. Takagi, K. Matsuzaki, N. Kasagi, Evaluation of SOFC anode polarization simulation using three-dimensional microstructures reconstructed by FIB tomography, Electrochim. Acta. 56 (2011) 4015-4021. doi:10.1016/j.electacta.2011.02.010.

[86] T. Shimura, Z. Jiao, N. Shikazono, Evaluation of nickel-yttria stabilized zirconia anode degradation during discharge operation and redox cycles operation by electrochemical calculation, J. Power Sources. 330 (2016) 149-155. doi:10.1016/j.jpowsour.2016.09.006.

[87] Y. Suzue, N. Shikazono, N. Kasagi, Micro modeling of solid oxide fuel cell anode based on stochastic reconstruction, J. Power Sources. 184 (2008) 52-59. doi:10.1016/j.jpowsour.2008.06.029.

[88] T.A. Prokop, K. Berent, H. Iwai, J.S. Szmyd, G. Brus, A three-dimensional heterogeneity analysis of electrochemical energy conversion in SOFC anodes using electron nanotomography and mathematical modeling, Int. J. Hydrogen Energy. 43 (2018) 1001610030. doi:10.1016/j.ijhydene.2018.04.023.

[89] P.R. Shearing, Q. Cai, J.I. Golbert, V. Yufit, C.S. Adjiman, N.P. Brandon, Microstructural analysis of a solid oxide fuel cell anode using focused ion beam techniques coupled with electrochemical simulation, J. Power Sources. 195 (2010) 4804-4810.

doi:10.1016/j.jpowsour.2010.02.047. 
[90] X. Lu, A. Bertei, T.M.M. Heenan, Y. Wu, D.J.L. Brett, P.R. Shearing, Multi-length scale microstructural design of micro-tubular Solid Oxide Fuel Cells for optimised power density and mechanical robustness, J. Power Sources. 434 (2019) 226744. doi:10.1016/j.jpowsour.2019.226744.

[91] A.J. Bard, L.R. Faulkner, Electrochemical methods: fundamentals and applications, Wiley, New York, 2001.

[92] A. Bertei, J. Mertens, C. Nicolella, Electrochemical simulation of planar solid oxide fuel cells with detailed microstructural modeling, Electrochim. Acta. 146 (2014) 151-163. doi:10.1016/j.electacta.2014.08.120.

[93] J. Mizusaki, K. Amano, S. Yamauchi, K. Fueki, Electrode reaction at Pt, O2(g)/stabilized zirconia interfaces. Part I: Theoretical consideration of reactio model, Solid State Ionics. 22 (1987) 313-322. doi:10.1016/0167-2738(87)90149-4.

[94] J. Mizusaki, Model for solid electrolyte gas electrode reaction kinetics; key concepts, basic model construction, extension of models, new experimental techniques for model confirmation, and future prospects, Electrochemistry. 82 (2014) 819-829. doi:10.5796/electrochemistry.82.819.

[95] K. Matsuzaki, N. Shikazono, N. Kasagi, Three-dimensional numerical analysis of mixed ionic and electronic conducting cathode reconstructed by focused ion beam scanning electron microscope, J. Power Sources. 196 (2011) 3073-3082. doi:10.1016/j.jpowsour.2010.11.142.

[96] K. Miyoshi, T. Miyamae, H. Iwai, M. Saito, M. Kishimoto, H. Yoshida, Exchange current model for ( $\mathrm{La} 0.8 \mathrm{Sr} 0.2) 0.95 \mathrm{MnO} 3$ (LSM) porous cathode for solid oxide fuel cells, J. Power Sources. 315 (2016) 63-69. doi:10.1016/j.jpowsour.2016.02.076.

[97] N. Shikazono, D. Kanno, K. Matsuzaki, H. Teshima, S. Sumino, N. Kasagi, Numerical assessment of SOFC anode polarization based on three-dimensional model microstructure reconstructed from FIB-SEM images, J. Electrochem. Soc. 157 (2010) B665-B672. doi:10.1149/1.3330568.

[98] M.E. Lynch, D. Ding, W.M. Harris, J.J. Lombardo, G.J. Nelson, W.K.S. Chiu, M. Liu, Flexible multiphysics simulation of porous electrodes: Conformal to 3D reconstructed microstructures, Nano Enérgy. 2 (2013) 105-115. doi:10.1016/j.nanoen.2012.08.002.

[99] M. Sabharwal, L.M. Pant, A. Putz, D. Susac, J. Jankovic, M. Secanell, Analysis of Catalyst Layer Microstructures: From Imaging to Performance, Fuel Cells. 16 (2016) 734-753. doi:10.1002/fuce.201600008.

[100] E.M. Ryan, P.P. Mukherjee, Mesoscale modeling in electrochemical devices-A critical perspective, Prog. Energy Combust. Sci. 71 (2019) 118-142.

doi:10.1016/j.pecs.2018.11.002. 
[101] G. Qiu, C.R. Dennison, K.W. Knehr, E.C. Kumbur, Y. Sun, Pore-scale analysis of effects of electrode morphology and electrolyte flow conditions on performance of vanadium redox flow batteries, J. Power Sources. 219 (2012) 223-234. doi:10.1016/j.jpowsour.2012.07.042.

[102] X. You, Q. Ye, P. Cheng, The Dependence of Mass Transfer Coefficient on the Electrolyte Velocity in Carbon Felt Electrodes: Determination and Validation, J. Electrochem. Soc. 164 (2017) E3386-E3394. doi:10.1149/2.0401711jes.

[103] S.K. Murthy, A.K. Sharma, C. Choo, E. Birgersson, Analysis of Concentration Overpotential in an All-Vanadium Redox Flow Battery, J. Electrochem. Soc. 165 (2018) A1746-A1752. doi:10.1149/2.0681809jes.

[104] L. Zielke, T. Hutzenlaub, D.R. Wheeler, C.W. Chao, I. Manke, A. Hilger, N. Paust, R. Zengerle, S. Thiele, Three-phase multiscale modeling of a LiCoO2 cathode: Combining the advantages of FIB-SEM imaging and X-ray tomography, Adv. Energy Mater. 5 (2015) 1-8. doi:10.1002/aenm.201401612.

[105] B.L. Trembacki, A.N. Mistry, D.R. Noble, M.E. Ferraro, P.P. Mukherjee, S.A. Roberts, Editors' Choice-Mesoscale Analysis of Conductive Binder Domain Morphology in Lithium-Ion Battery Electrodes, J. Electrochem. Soc. 165 (2018) E725-E736. doi:10.1149/2.0981813jes.

[106] F.L.E. Usseglio-Viretta, A. Colclasure, A.N. Mistry, K.P.Y. Claver, F. Pouraghajan, D.P. Finegan, T.M.M. Heenan, D. Abraham, P.P. Mukherjee, D. Wheeler, P. Shearing, S.J. Cooper, K. Smith, Resolving the Discrepancy in Tortuosity Factor Estimation for Li-Ion Battery Electrodes through Micro-Macro Modeling and Experiment, J. Electrochem. Soc. 165 (2018) A3403-A3426. doi:10.1149/2.0731814jes.

[107] L. Wu, X. Xiao, Y. Wen, J. Zhang, Three-dimensional finite element study on stress generation in synchrotron $X$-ray tomography reconstructed nickel-manganese-cobalt based half cell, J. Power Sources. 336 (2016) 8-18. doi:10.1016/j.jpowsour.2016.10.052.

[108] Y. Zeng, R.B. Smith, P. Bai, M.Z. Bazant, Simple formula for Marcus-Hush-Chidsey kinetics, J. Electroanal. Chem. 735 (2014) 77-83. doi:10.1016/j.jelechem.2014.09.038.

[109] T. Danner, M. Singh, S. Hein, J. Kaiser, H. Hahn, A. Latz, Thick electrodes for Li-ion batteries: A model based analysis, J. Power Sources. 334 (2016) 191-201. doi:10.1016/j.jpowsour.2016.09.143.

[110] A.G. Kashkooli, E. Foreman, S. Farhad, D.U. Lee, K. Feng, G. Lui, V. De Andrade, Z. Chen, Morphological and Electrochemical Characterization of Nanostructured Li 4 Ti 5 O 12 Electrodes Using Multiple Imaging Mode Synchrotron X-ray Computed Tomography, J. Electrochem. Soc. 164 (2017) A2861-A2871. doi:10.1149/2.0101713jes. 
[111] S. Lee, A.M. Sastry, J. Park, Study on microstructures of electrodes in lithium-ion batteries using variational multi-scale enrichment, J. Power Sources. 315 (2016) 96-110. doi:10.1016/j.jpowsour.2016.02.086.

[112] L. Kang, W. Fenghui, Z. Xiang, L. Yongjun, Impact of composite particle morphology on the performance of solid oxide fuel cell electrode: A study by finite element modelling, Int. J. Electrochem. Sci. 11 (2016) 1382-1394.

[113] S. Sunde, Monte Carlo Simulations of Polarization Resistance of Composite Electrodes for Solid Oxide Fuel Cells, J. Electrochem. Soc. 143 (2006) 1930. doi:10.1149/1.1836927.

[114] Özden Çelikbilek, D. Jauffrès, E. Siebert, L. Dessemond, M. Burriel, C.L. Martin, E. Djurado, Rational design of hierarchically nanostructured electrodes for solid oxide fuel cells, J. Power Sources. 333 (2016) 72-82. doi:10.1016/j.jpowsour.2016.09.156.

[115] T. Carraro, J. Joos, B. Rüger, A. Weber, E. Ivers-Tiffée, 3D finite element model for reconstructed mixed-conducting cathodes: I. Performance quantification, Electrochim. Acta. 77 (2012) 315-323. doi:10.1016/j.electacta.2012.04.109.

[116] A. Häffelin, J. Joos, M. Ender, A. Weber, E. Ivers-Tiffée, Timé-Dependent 3D Impedance Model of Mixed-Conducting Solid Oxide Fuel Cell Cathodes, J. Electrochem. Soc. 160 (2013) F867-F876. doi:10.1149/2.093308jes.

[117] C.R. Birkl, E. McTurk, M.R. Roberts, P.G. Bruce, D.A. Howey, A Parametric Open Circuit Voltage Model for Lithium Ion Batteries, J. Electrochem. Soc. 162 (2015) A2271-A2280. doi:10.1149/2.0331512jes.

[118] R. Fang, P. Farah, A. Popp, W.A. Wall, A monolithic, mortar-based interface coupling and solution scheme for finite element simulations of lithium-ion cells, Int. J. Numer. Methods Eng. 114 (2018) 1411-1437. doi:10.1002/nme.5792.

[119] M.Z. Bazant, Theory of chemical kinetics and charge transfer based on nonequilibrium thermodynamics, Acc. Chem. Res. 46 (2013) 1144-1160. doi:10.1021/ar300145c.

[120] D.A. Cogswell, M.Z. Bazant, Size-dependent phase morphologies in LiFePO4 battery particles, Electrochem. Commun. 95 (2018) 33-37. doi:10.1016/j.elecom.2018.08.015.

[121] G.K. Singh, G. Ceder, M.Z. Bazant, Intercalation dynamics in rechargeable battery materials: General theory and phase-transformation waves in LiFePO4, Electrochim. Acta. 53 (2008) 7599-7613. doi:10.1016/j.electacta.2008.03.083.

[122] M.Z. Bazant, Thermodynamic stability of driven open systems and control of phase separation by electro-autocatalysis, Faraday Discuss. 199 (2017) 423-463. doi:10.1039/c7fd00037e.

[123] H.C. Yu, M.J. Choe, G.G. Amatucci, Y.M. Chiang, K. Thornton, Smoothed Boundary Method for simulating bulk and grain boundary transport in complex polycrystalline 
microstructures, Comput. Mater. Sci. 121 (2016) 14-22.

doi:10.1016/j.commatsci.2016.04.028.

[124] S.J. Cooper, A. Bertei, D.P. Finegan, N.P. Brandon, Simulated impedance of diffusion in porous media, Electrochim. Acta. 251 (2017) 681-689. doi:10.1016/j.electacta.2017.07.152.

[125] W. Zheng, S.H. Kim, A multiscale approach to accelerate pore-scale simulation of porous electrodes, J. Power Sources. 348 (2017) 21-29. doi:10.1016/j.jpowsour.2017.02.073.

[126] O. C. Zienkiewicz, R. L. Taylor, The Finite Element Method - Volume 1: The Basis, Fifth edit, Butterworth Heinemann, Oxford, 2000.

[127] Comsol, Comsol Multiphysics User's Guide, Burlington, MA, 2012. doi:10.1016/S02608774(99)00111-9.

[128] B. Rüger, A. Weber, E. Ivers-Tiffee, 3D-Modelling and Performance Evaluation of Mixed Conducting (MIEC) Cathodes, ECS Trans. 7 (2007) 2065-2074. doi:10,1149/1.2729320.

[129] C.R. Kreller, M.E. Drake, S. Adler, Influence of Electrode Morphology on Electrochemical Response of SOFC Cathodes, ECS Trans. 28 (2010) 105-121. doi:10.1149/1.3495836.

[130] C. Kreller, M. Drake, S.B. Adler, H.-Y. Chen, H.-C. Yu, K. Thornton, J.R. Wilson, S.A. Barnett, Modeling SOFC Cathodes Based on 3-D Representations of Electrode Microstructure, ECS Trans. 35 (2011) 815-822. doi:10.1149/1.3570062.

[131] G.J. Nelson, A. Nakajo, B.N. Cassenti, M.B. Degostin, K.R. Bagshaw, A.A. Peracchio, G. Xiao, S. Wang, F. Chen, W.K.S. Chiu, A rapid analytical assessment tool for three dimensional electrode microstructural networks with geometric sensitivity, J. Power Sources. 246 (2014) 322-334. doi:10.1016/j.jpowsour.2013.07.009.

[132] A. Bertei, V. Yufit, F. Tariq, N.P. Brandon, A novel approach for the quantification of inhomogeneous 3D current distribution in fuel cell electrodes, J. Power Sources. 396 (2018) 246-256. doi:10.1016/j.jpowsour.2018.06.029.

[133] M. Wang, X. Xiao, X. Huang, A multiphysics microstructure-resolved model for silicon anode lithium-ion batteries, J. Power Sources. 348 (2017) 66-79. doi:10.1016/j.jpowsour.2017.02.037.

[134] M.M. Forouzan, M. Wray, L. Robertson, D.R. Wheeler, Tortuosity of Composite Porous Electrodes with Various Conductive Additives in an Alkaline System, J. Electrochem. Soc. 164 (2017) A3117-A3130. doi:10.1149/2.0911713jes.

[135] S. Celik, B. Ibrahimoglu, M.D. Mat, Y. Kaplan, T.N. Veziroglu, Micro level two dimensional stress and thermal analysis anode/electrolyte interface of a solid oxide fuel cell, Int. J. Hydrogen Energy. 40 (2015) 7895-7902. doi:10.1016/j.ijhydene.2014.10.057. 
[136] A. Utz, J. Joos, A. Weber, E. Ivers-Tiffée, Electrochemical Oxidation at SOFC Anodes: Comparison of Patterned Nickel Anodes and Nickel/8YSZ Cermet Anodes, in: ECS Trans., The Electrochemical Society, 2011: pp. 1669-1682. doi:10.1149/1.3570154.

[137] B. Rüger, J. Joos, A. Weber, T. Carraro, E. Ivers-Tiffée, 3D Electrode Microstructure Reconstruction and Modelling, ECS Trans. 25 (2009) 1211-1220. doi:10.1149/1.3205650.

[138] W. Bangerth, R. Hartmann, G. Kanschat, deal.II---A general-purpose object-oriented finite element library, ACM Trans. Math. Softw. 33 (2007) 24-es. doi:10.1145/1268776.1268779.

[139] T. Carraro, J. Joos, B. Rüger, A. Weber, E. Ivers-Tiffée, 3D finite element model for reconstructed mixed-conducting cathodes: II. Parameter sensitivity analysis, Electrochim. Acta. 77 (2012) 309-314. doi:10.1016/j.electacta.2012.04.163.

[140] S.A. Roberts, H. Mendoza, V.E. Brunini, B.L. Trembacki, D.R. Noble, A.M. Grillet, Insights Into Lithium-Ion Battery Degradation and Safety Mechanisms From Mesoscale Simulations Using Experimentally Reconstructed Mesostructures, J. Electrochem. Energy Convers. Storage. 13 (2016) 031005. doi:10.1115/1.4034410.

[141] H. Mendoza, S.A. Roberts, V.E. Brunini, A.M. Grillet, Mechanical and Electrochemical Response of a LiCoO2 Cathode using Reconstructed Microstructures, Electrochim. Acta. 190 (2016) 1-15. doi:10.1016/j.electacta.2015.12.224.

[142] E.L. Reinholz, S.A. Roberts, C.A. Apblett, J.B. Lechman, P.R. Schunk, Composition and Manufacturing Effects on Electrical Conductivity of Li/FeS 2 Thermal Battery Cathodes, J. Electrochem. Soc. 163 (2016) A1723-A1729. doi:10.1149/2.1191608jes.

[143] S.A. Roberts, V.E. Brunini, K.N. Long, A.M. Grillet, A framework for three-dimensional mesoscale modeling of anisotropic swelling and mechanical deformation in lithium-ion electrodes, J. Electrochem. Soc. 161 (2014) F3052-F3059. doi:10.1149/2.0081411jes.

[144] M. Smith, R.E. García, Q.C. Horn, The Effect of Microstructure on the Galvanostatic Discharge of Graphite Anode Electrodes in LiCoO[sub 2]-Based Rocking-Chair Rechargeable Batteries, J. Electrochem. Soc. 156 (2009) A896. doi:10.1149/1.3216000.

[145] S. Celik, B. Ibrahimoglu, S. Toros, M.D. Mat, Three dimensional stress analysis of solid oxide fuel cell anode micro structure, Int. J. Hydrogen Energy. 39 (2014) 19119-19131. doi:10.1016/j.ijhydene.2014.09.110.

[146] T. Zhang, M. Kamlah, A nonlocal species concentration theory for diffusion and phase changes in electrode particles of lithium ion batteries, Contin. Mech. Thermodyn. 30 (2018) 553-572. doi:10.1007/s00161-018-0624-z.

[147] S. Simaafrookhteh, M. Shakeri, M. Baniassadi, A. Alizadeh Sahraei, Microstructure reconstruction and characterization of the porous GDLs for PEMFC based on fibers orientation distribution, Fuel Cells. 18 (2018) 160-172. doi:10.1002/fuce.201700239. 
[148] Y. Hu, Q. Zhou, X. Gao, A. Jacobson, D. Zorin, D. Panozzo, Tetrahedral meshing in the wild, ACM Trans. Graph. 37 (2018) 60. doi:10.1145/3197517.3201353.

[149] S.A. Roberts, H. Mendoza, V.E. Brunini, D.R. Noble, A verified conformal decomposition finite element method for implicit, many-material geometries, J. Comput. Phys. 375 (2018) 352-367. doi:10.1016/j.jcp.2018.08.022.

[150] R. Fang, M. Kronbichler, M. Wurzer, W.A. Wall, Parallel, physics-oriented, monolithic solvers for three-dimensional, coupled finite element models of lithium-ion cells, Comput. Methodes Appl. Mech. Eng. 350 (2019) 803-835. doi:10.1016/j.cma.2019.03.017.

[151] S.J. Cooper, D.S. Eastwood, J. Gelb, G. Damblanc, D.J.L. Brett, R.S. Bradley, P.J. Withers, P.D. Lee, A.J. Marquis, N.P. Brandon, P.R. Shearing, Image based modelling of microstructural heterogeneity in LiFePO 4 electrodes for Li-ion batteries, J. Power Sources. 247 (2014) 1033-1039. doi:10.1016/j.jpowsour.2013.04.156.

[152] M. Neumann, J. Staněk, O.M. Pecho, L. Holzer, V. Beneš, V. Schmidt, Stochastic 3D modeling of complex three-phase microstructures in SOFC-electrodes with completely connected phases, Comput. Mater. Sci. 118 (2016) 353-364. doi:10.1016/j.commatsci.2016.03.013.

[153] G. Rinaldi, A. Nakajo, J. Van herle, P. Burdet, E. Oveisi, M. Cantoni, Strontium Migration at the GDC-YSZ Interface of Solid Oxide Cells in SOFC and SOEC Modes, ECS Trans. 78 (2017) 3297-3307. doi:10.1149/07801.3297ecst.

[154] Z. Chen, X. Wang, F. Giuliani, A. Atkinson, Analyses of microstructural and elastic properties of porous SOFC cathodes based on focused ion beam tomography, J. Power Sources. 273 (2015) 486-494. doi:10.1016/j.jpowsour.2014.09.131.

[155] S. Sunde, Monte Carlo Simulations of Conductivity of Composite Electrodes for Solid Oxide Fuel Cells, J. Electrochem. Soc. 143 (2006) 1123. doi:10.1149/1.1836595.

[156] S. Sunde, Calculations of impedance of composite anodes for solid oxide fuel cells, Electrochim. Acta. 42 (1997) 2637-2648. doi:10.1016/S0013-4686(96)00455-0.

[157] L.C.R. Schneider, C.L. Martin, Y. Bultel, D. Bouvard, E. Siebert, Discrete modelling of the electrochemical performance of SOFC electrodes, Electrochim. Acta. 52 (2006) 314-324. doi:10.1016/j.electacta.2006.05.018.

[158] S. Hou, X. Shan, Q. Zou, G.D. Doolen, W.E. Soll, Evaluation of two lattice Boltzmann models for multiphase flows, J. Comput. Phys. 138 (1997) 695-713. doi:10.1006/jcph.1997.5839.

[159] C.C. Chueh, M. Secanell, W. Bangerth, N. Djilali, Multi-level adaptive simulation of transient two-phase flow in heterogeneous porous media, Comput. Fluids. 39 (2010) 15851596. doi:10.1016/j.compfluid.2010.05.011. 
[160] J. Donea, A. Huerta, J.-P. Ponthot, A. Rodriguez-Ferran, Arbitrary lagrangian-eulerian methods, in: E. Stein, R. de Borst, T.J.R. Hughes (Eds.), Encycl. Comput. Mech. Vol. 1 Fundam., John Wiley \& Sons, 2004.

[161] K. Shah, N. Balsara, S. Banerjee, M. Chintapalli, A.P. Cocco, W.K.S. Chiu, I. Lahiri, S. Martha, A. Mistry, P.P. Mukherjee, V. Ramadesigan, C.S. Sharma, V.R. Subramanian, S. Mitra, A. Jain, State of the art and future research needs for multiscale analysis of Li-ion cells, J. Electrochem. Energy Convers. Storage. 14 (2017) 020801. doi:10.1115/1.4036456.

[162] R.J. LeVeque, Finite Volume Methods for Hyperbolic Problems, Cambridge University Press, Cambridge, 2010. doi:10.1017/cbo9780511791253.

[163] W. Versteeg, H.K. and Malalasekera, An introduction to computational fluid dynamics: the finite volume method. Pearson Education., 2nd ed., Pearson Education Ltd, Harlow, 2007.

[164] F. Wang, X. Li, Pore-Scale Simulations of Porous Electrodes of Li-O2 Batteries at Different Saturation Levels, ACS Appl. Mater. Interfaces. 10 (2018) 26222-26232. doi:10.1021/acsami.8b06624.

[165] K. Miyawaki, M. Kishimoto, H. Iwai, M. Saito, H. Yoshida, Comprehensive understanding of the active thickness in solid oxide fuel cell anodes using experimental, numerical and semi-analytical approach, J. Power Sourcés. 267 (2014) 503-514. doi:10.1016/j.jpowsour.2014.05.112.

[166] K. Miyoshi, H. Iwai, M. Kishimoto, M. Saito, H. Yoshida, Chromium poisoning in (La,Sr)MnO3 cathode: Three-dimensional simulation of a solid oxide fuel cell, J. Power Sources. 326 (2016) 331-340. doi:10.1016/j.jpowsour.2016.06.110.

[167] Q. Cai, C.S. Adjiman, N.P. Brandon, Investigation of the active thickness of solid oxide fuel cell electrodes using a 3D microstructure model, Electrochim. Acta. 56 (2011) 10809-10819. doi:10.1016/j.electacta.2011.06.105.

[168] M. Kishimoto, H. Iwai, M. Saito, H. Yoshida, Three-dimensional simulation of SOFC anode polarization characteristics based on sub-grid scale modeling of microstructure, $\mathrm{J}$. Electrochem. Soc. 159 (2012) B315-B323. doi:10.1149/2.086203jes.

[169] M. Kishimoto, K. Miyawaki, H. Iwai, M. Saito, H. Yoshida, Effect of composition ratio of Ni-YSZ anode on distribution of effective three-phase boundary and power generation performance, Fuel Cells. 13 (2013) 476-486. doi:10.1002/fuce.201200174.

[170] D.A.W. Gawel, J.G. Pharoah, S.B. Beale, Development of a SOFC performance model to analyze the powder to power performance of electrode microstructures, ECS Trans. 68 (2015) 1979-1987. doi:10.1149/06801.1979ecst.

[171] M. Finsterbusch, T. Danner, C.L. Tsai, S. Uhlenbruck, A. Latz, O. Guillon, High Capacity Garnet-Based All-Solid-State Lithium Batteries: Fabrication and 3D-Microstructure 
Resolved Modeling, ACS Appl. Mater. Interfaces. 10 (2018) 22329-22339. doi:10.1021/acsami.8b06705.

[172] G.B. Less, J.H. Seo, S. Han, A.M. Sastry, J. Zausch, A. Latz, S. Schmidt, C. Wieser, D. Kehrwald, S. Fell, Micro-Scale Modeling of Li-Ion Batteries: Parameterization and Validation, J. Electrochem. Soc. 159 (2012) A697-A704. doi:10.1149/2.096205jes.

[173] J. Golbert, C.S. Adjiman, N.P. Brandon, Microstructural modeling of solid oxide fuel cell anodes, Ind. Eng. Chem. Res. 47 (2008) 7693-7699. doi:10.1021/ie800065w.

[174] K.J. Lange, P.C. Sui, N. Djilali, Determination of effective transport properties in a PEMFC catalyst layer using different reconstruction algorithms, J. Power Sources. 208 (2012) 354365. doi:10.1016/j.jpowsour.2011.11.001.

[175] K.J. Lange, P.C. Sui, N. Djilali, Pore scale modeling of a proton exchange membrane fuel cell catalyst layer: Effects of water vapor and temperature, J. Power Sources. 196 (2011) 3195-3203. doi:10.1016/j.jpowsour.2010.11.118.

[176] E. Monaco, Microphase lattice Boltzmann simulation of microfluidics, (2011).

[177] Y. Peng, S. Laura, Equations of state in a lattice Boltzmann mơdel, Phys. Fluids. 18 (2006) 042101. doi:10.1063/1.2187070.

[178] Y.H. Qian, D. Dhumieres, P. Lallemand, Lattice Bgk Models for Navier-Stokes Equation, Europhys. Lett. 17 (1992) 479-484. doi:10.1209/0295-5075/17/6/001.

[179] D.H. Jeon, D.Es.P. Inc, Lattice Boltzmann Simulation on Water Transport in Gas Diffusion Layer of Polymer Electrolyte Membrane Fuel Cells, 2016 Int. Conf. Comput. Model. Simul. Appl. Math. (Cmsam 2016). 195 (2016) 22-25. doi:10.12783/dtcse/cmsam2016/3545.

[180] G.R. Molaeimanesh, M.H. Akbari, A three-dimensional pore-scale model of the cathode electrode in polymer-electrolyte membrane fuel cell by lattice Boltzmann method, J. Power Sources. 258 (2014) 89-97. doi:10.1016/j.jpowsour.2014.02.027.

[181] L. Chen, R. Zhang, P.,He, Q. Kang, Y.L. He, W.Q. Tao, Nanoscale simulation of local gas transport in catalyst layers of proton exchange membrane fuel cells, J. Power Sources. 400 (2018) 114-125. doi:10.1016/j.jpowsour.2018.07.099.

[182] D. Zhang, A. Forner-Cuenca, O.. Taiwo, V. Yufit, F.. Brushett, N.P. Brandon, S. Gu, Q. Cai, Understanding the role of porous electrodes microstructure in redox flow battery performance using an experimentally validated 3D pore-scale lattice Boltzmann model, J. Power Sources. (2019) Submitted.

[183] Z.Y. Jiang, Z.G. Qu, L. Zhou, W.Q. Tao, A microscopic investigation of ion and electron transport in lithium-ion battery porous electrodes using the lattice Boltzmann method, Appl. Energy. 194 (2016) 530-539. doi:10.1016/j.apenergy.2016.10.125. 
[184] Z.Y. Jiang, Z.G. Qu, L. Zhou, Lattice Boltzmann simulation of ion and electron transport during the discharge process in a randomly reconstructed porous electrode of a lithium-ion battery, Int. J. Heat Mass Transf. 123 (2018) 500-513. doi:10.1016/j.ijheatmasstransfer.2018.03.004.

[185] D. Wiedenmann, L. Keller, L. Holzer, J. Stojadinović, B. Münch, L. Suarez, B. Fumey, H. Hagendorfer, R. Brönnimann, P. Modregger, M. Gorbar, U.F. Vogt, A. Züttel, F. La Mantia, R. Wepf, B. Grobéty, Three-dimensional pore structure and ion conductivity of porous ceramic diaphragms, AIChE J. 59 (2013) 1446-1457. doi:10.1002/aic.14094.

[186] L. Holzer, B. Iwanschitz, T. Hocker, L. Keller, O. Pecho, G. Sartoris, P. Gasser, B. Muench, Redox cycling of Ni-YSZ anodes for solid oxide fuel cells: Influence of tortuosity, constriction and percolation factors on the effective transport properties, J. Power Sources. 242 (2013) 179-194. doi:10.1016/j.jpowsour.2013.05.047.

[187] H.W. Choi, A. Berson, J.G. Pharoah, S.B. Beale, Effective transport/properties of the porous electrodes in solid oxide fuel cells, Proc. Inst. Mech. Eng. Part AJ. Power Energy. 225 (2011) 183-197. doi:10.1177/2041296710394266.

[188] N.S.K. Gunda, H.W. Choi, A. Berson, B. Kenney, K. Karan, J.G. Pharoah, S.K. Mitra, Focused ion beam-scanning electron microscopy on solid-oxide fuel-cell electrode: Image analysis and computing effective transport properties, J. Power Sources. 196 (2011) 35923603. doi:10.1016/j.jpowsour.2010.12.042.

[189] W.M. Harris, W.K.S. Chiu, Determining the representative volume element size for threedimensional microstructural material charactérization. Part 1: Predictive models, J. Power Sources. 282 (2015) 552-561. doi:10.1016/j.jpowsour.2015.02.035.

[190] W.M. Harris, W.K.S. Chiu, Determining the representative volume element size for threedimensional microstructural material characterization. Part 2: Application to experimental data, J. Power Sources. 282 (2015) 622-629. doi:10.1016/j.jpowsour.2015.02.052.

[191] D. Roussel, A. Lichtner, D. Jauffrès, R.K. Bordia, C.L. Martin, Effective transport properties of 3D multi-component microstructures with interface resistance, Comput. Mater. Sci. 96 (2015) 277-283. doi:10.1016/j.commatsci.2014.09.027.

[192] M.B. Degostin, A. Nakajo, B.N. Cassenti, A.A. Peracchio, G.J. Nelson, W.K.S. Chiu, Geometric sensitivity of electrochemical fin shape on three dimensional microstructure network conductivity analysis, J. Power Sources. 291 (2015) 181-194. doi:10.1016/j.jpowsour.2015.04.153.

[193] A. Nakajo, A.M. Kiss, A.P. Cocco, W.M. Harris, M.B. DeGostin, F. Greco, G.J. Nelson, A.A. Peracchio, B.N. Cassenti, A. Deriy, S. Wang, Y. Chen-Wiegart, J. Wang, J. Van herle, W.K.S. Chiu, Characterization of Cracks and their Effects on the Effective Transport 
Pathways in Ni-YSZ Anodes after Reoxidation Using X-Ray Nanotomography, ECS Trans. 68 (2015) 1069-1081. doi:10.1149/06801.1069ecst.

[194] 3D Visualization \& Analysis Software, (n.d.).

https://www.thermofisher.com/uk/en/home/industrial/electron-microscopy/electronmicroscopy-instruments-workflow-solutions/3d-visualization-analysis-software.html.

[195] Z. Chen, X. Wang, A. Atkinson, N. Brandon, Journal of the European Ceramic Society Spherical indentation of porous ceramics : Cracking and toughness, J. Eur. Ceram. Soc. 36 (2016) 3473-3480. doi:10.1016/j.jeurceramsoc.2016.05.010.

[196] R. Clague, P.R. Shearing, P.D. Lee, Z. Zhang, D.J.L. Brett, A.J. Marquis, N.P. Brandon, Stress analysis of solid oxide fuel cell anode microstructure reconstructed from focused ion beam tomography, J. Power Sources. 196 (2011) 9018-9021. doi:10.1016/j.jpowsour.2010.12.097.

[197] B. Song, E. Ruiz-Trejo, A. Bertei, N.P. Brandon, Quantification of the degradation of NiYSZ anodes upon redox cycling, J. Power Sources. 374 (2018) 61-68. doi:10.1016/j.jpowsour.2017.11.024.

[198] L.C.R. Schneider, C.L. Martin, Y. Bultel, L. Dessemond, D. Bouvard, Percolation effects in functionally graded SOFC electrodes, Electrochim. Acta. 52 (2007) 3190-3198. doi:10.1016/j.electacta.2006.09.071.

[199] G.J. Nelson, A.A. Peracchio, W.K.S. Chiu, Analytical investigations of varying cross section microstructures on charge transfer in solid oxide fuel cell electrodes, J. Power Sources. 196 (2011) 4695-4704. doi:10.1016/j.jpowsour.2010.12.103.

[200] A. Banerjee, O. Deutschmann, Elementary kinetics of the oxygen reduction reaction on LSM-YSZ composite cathodes, J. Catal. 346 (2017) 30-49. doi:10.1016/j.jcat.2016.11.035.

[201] M. Gong, R.S. Gemmen, X. Liu, Modeling of oxygen reduction mechanism for 3PB and 2PB pathways at solid oxide fuel cell cathode from multi-step charge transfer, J. Power Sources. 201 (2012) 204-218. doi:10,1016/j.jpowsour.2011.11.002.

[202] A. Bertei, M.P. Carpanese, D. Clematis, A. Barbucci, M.Z. Bazant, C. Nicolella, Understanding the electrochemical behaviour of LSM-based SOFC cathodes. Part II Mechanistic modelling and physically-based interpretation, Solid State Ionics. 303 (2017) 181-190. doi:10.1016/j.ssi.2016.09.028.

[203] Z. Jiao, T. Shimura, N. Shikazono, Numerical assessment of SOFC anode polarization with microstructure evolution, ECS Trans. 68 (2015) 1281-1289. doi:10.1149/06801.1281ecst.

[204] S.B. Adler, J.A. Lane, B.C.H. Steele, Electrode kinetics of porous mixed-conducting oxygen electrodes, J. Electrochem. Soc. 143 (1996) 3554-3564. doi:10.1149/1.1837252. 
[205] Y.C.K. Chen-Wiegart, R. Demike, C. Erdonmez, K. Thornton, S.A. Barnett, J. Wang, Tortuosity characterization of 3D microstructure at nano-scale for energy storage and conversion materials, J. Power Sources. 249 (2014) 349-356. doi:10.1016/j.jpowsour.2013.10.026.

[206] A. Nakajo, A.P. Cocco, M.B. DeGostin, A.A. Peracchio, B.N. Cassenti, M. Cantoni, J. Van herle, W.K.S. Chiu, Accessible triple-phase boundary length: A performance metric to account for transport pathways in heterogeneous electrochemical materials, J. Power Sources. 325 (2016) 786-800. doi:10.1016/j.jpowsour.2016.06.046.

[207] A. Nakajo, A.P. Cocco, M.B. DeGostin, P. Burdet, A.A. Peracchio, B.N. Cassenti, M. Cantoni, J. Van herle, W.K.S. Chiu, Evolution of 3-D Transport Pathways and Triple-Phase Boundaries in the Ni-YSZ Hydrogen Electrode upon Fuel Cell or Electrolysis Cell Operation, ECS Trans. 78 (2017) 3205-3215. doi:10.1149/07801.3205ecst.

[208] W. He, J.S. Yi, T. Van Nguyen, Two-phase flow model of the cathode of PEM fuel cells using interdigitated flow fields, AIChE J. 46 (2000) 2053-2064. doi:10.1002/aic.690461016.

[209] D. Natarajan, T. Van Nguyen, A Two-Dimensional, Two-Phasé, Multicomponent, Transient Model for the Cathode of a Proton Exchange Membrane Fuel Cell Using Conventional Gas Distributors [Journal of the Electrochemical Society 148, A1324 (2001)], J. Electrochem. Soc. 150 (2003) L5. doi:10.1149/1.1554417.

[210] T. V Nguyen, D. Natarajan, Three dimensional effects of liquid water flooding in the cathode of a PEM fuel cell, J. Power Sources. 115 (2003) 66-80. doi:org/10.1016/S03787753(02)00624-9.

[211] G. Lin, T. Van Nguyen, A Two-Dimensional Two-Phase Model of a PEM Fuel Cell, J. Electrochem. Soc. 153 (2006) A372. doi:10.1149/1.2142267.

[212] Q. Ye, T. Van Nguyen, Three-Dimensional Simulation of Liquid Water Distribution in a PEMFC with Experimentally Measured Capillary Functions, J. Electrochem. Soc. 154 (2007) B1242. doi:10.1149/1.2783775.

[213] K. Ahmed, On Modeling Water Transport in Polymer Electrolyte Membrane Fuel Cell, Open Access Biostat. Bioinforma. 1 (2018) 6913-6927. doi:10.31031/oabb.2018.01.000503.

[214] H. Wua, P. Berg, X. Li, Steady and unsteady 3D non-isothermal modeling of PEM fuel cells with the effect of non-equilibrium phase transfer, Appl. Energy. 87 (2010) 2778-2784. doi:10.1016/j. apenergy.2009.06.024.

[215] Z.H. Wang, C.Y. Wang, Two-Phase Flow and Transport in the Interdigitated Air Cathode of Proton Exchange Membrane Fuel Cells, Proc. ASME Heat Transf. Div. 94 (2000) 27-33.

[216] H. Meng, C.-Y. Wang, Model of Two-Phase Flow and Flooding Dynamics in Polymer Electrolyte Fuel Cells, J. Electrochem. Soc. 152 (2006) A1733. doi:10.1149/1.1955007. 
[217] Y. Wang, C.-Y. Wang, A Nonisothermal, Two-Phase Model for Polymer Electrolyte Fuel Cells, J. Electrochem. Soc. 153 (2007) A1193. doi:10.1149/1.2193403.

[218] Y. Wang, C.-Y. Wang, Two-Phase Transients of Polymer Electrolyte Fuel Cells, J. Electrochem. Soc. 154 (2007) B636. doi:10.1149/1.2734076.

[219] J. Li, C.-Y. Wang, S. Basu, Two-phase flow and maldistribution in gas channels of a polymer electrolyte fuel cell, J. Power Sources. 187 (2009) 431-443. doi:10.1016/j.jpowsour.2008.11.039.

[220] S. Basu, C.-Y. Wang, K.S. Chen, Two-Phase Flow Maldistribution and Mitigation in Polymer Electrolyte Fuel Cells, J. Fuel Cell Sci. Technol. 6 (2009) 031007. doi:10.1115/1.2971124.

[221] W. Sun, B.A. Peppley, K. Karan, An improved two-dimensional agglomerate cathode model to study the influence of catalyst layer structural parameters, Electrochim. Acta. 50 (2005) 3359-3374. doi:10.1016/j.electacta.2004.12.009.

[222] R. Singh, A.R. Akhgar, P.C. Sui, K.J. Lange, N. Djilali, Dual-Beam FIB/SEM Characterization, Statistical Reconstruction, and Pore Scale Modeling of a PEMFC Catalyst Layer, J. Electrochem. Soc. 161 (2014) F415-F424. doi:10.1149/2.036404jes.

[223] H. Fathi, A. Raoof, S.H. Mansouri, M.T. van Genuchten, Effects of Porosity and Water Saturation on the Effective Diffusivity of a Cathode Catalyst Layer, J. Electrochem. Soc. 164 (2017) F298-F305. doi:10.1149/2.0871704jes.

[224] H. Fathi, A. Raoof, S.H. Mansouri, Insights into the role of wettability in cathode catalyst layer of proton exchange membrane fuel cell; pore scale immiscible flow and transport processes, J. Power Sources. 349 (2017) 57-67. doi:10.1016/j.jpowsour.2017.03.012.

[225] X. Zhang, Y. Gao, H. Ostadi, K. Jiang, R. Chen, Method to improve catalyst layer model for modelling proton exchange membrane fuel cell, J. Power Sources. 289 (2015) 114-128. doi:10.1016/j.jpowsour.2015.04.152.

[226] F.C. Cetinbas, S.G. Advani, A.K. Prasad, A Modified Agglomerate Model with Discrete Catalyst Particles for the PEM Fuel Cell Catalyst Layer, J. Electrochem. Soc. 160 (2013) F750-F756. doi:10.1149/2.017308jes.

[227] F.C. Cetinbas, S.G. Advani, A.K. Prasad, An Improved Agglomerate Model for the PEM Catalyst Layer with Accurate Effective Surface Area Calculation Based on the SpherePacking Approach, J. Electrochem. Soc. 161 (2014) F803-F813. doi:10.1149/2.116406jes.

[228] T. Sousa, C.M. Rangel, Pore scale modelling of a cathode catalyst layer in fuel cell environment: agglomerate reconstruction and variables optimization, J. Solid State Electrochem. 20 (2016) 541-554. doi:10.1007/s10008-015-3076-4. 
[229] S. Kamarajugadda, S. Mazumder, Generalized flooded agglomerate model for the cathode catalyst layer of a polymer electrolyte membrane fuel cell, J. Power Sources. 208 (2012) 328-339. doi:10.1016/j.jpowsour.2012.02.063.

[230] S.P. Kuttanikkad, M. Prat, J. Pauchet, Pore-network simulations of two-phase flow in a thin porous layer of mixed wettability: Application to water transport in gas diffusion layers of proton exchange membrane fuel cells, J. Power Sources. 196 (2011) 1145-1155. doi:10.1016/j.jpowsour.2010.09.029.

[231] K.J. Lee, J.H. Kang, J.H. Nam, Liquid water distribution in hydrophobic gas-diffusion layers with interconnect rib geometry: An invasion-percolation pore network analysis, Int. J. Hydrogen Energy. 39 (2014) 6646-6656. doi:10.1016/j.ijhydene.2014.01.206.

[232] R. Wu, Q. Liao, X. Zhu, H. Wang, Impacts of the mixed wettability on liquid water and reactant gas transport through the gas diffusion layer of proton exchange membrane fuel cells, Int. J. Heat Mass Transf. 55 (2012) 2581-2589. doi:10.1016/j.ijheatmasstransfer.2012.01.002.

[233] J. Ma, X. Zhang, Z. Jiang, H. Ostadi, K. Jiang, R. Chen, Flow properties of an intact MPL from nano-tomography and pore network modelling, Fuel. 136 (2014) 307-315. doi:10.1016/j.fuel.2014.07.040.

[234] X. Zhang, Y. Gao, H. Ostadi, K. Jiang, R. Chen, Modelling water intrusion and oxygen diffusion in a reconstructed microporous layer of PEM fuel cells, Int. J. Hydrogen Energy. 39 (2014) 17222-17230. doi:10.1016/j.ijhydene.2014.08.027.

[235] M. Fazeli, J. Hinebaugh, Z. Fishman, C. Tötzke, W. Lehnert, I. Manke, A. Bazylak, Pore network modeling to explore the effects of compression on multiphase transport in polymer electrolyte membrane fuel cell gas diffusion layers, J. Power Sources. 335 (2016) 162-171. doi:10.1016/j.jpowsour.2016.10.039.

[236] B. Straubhaar, J. Pauchet, M. Prat, International Journal of Heat and Mass Transfer Pore network modelling of condensation in gas diffusion layers of proton exchange membrane fuel cells, Int. J. Heat Mass Transf. 102 (2016) 891-901. doi:10.1016/j.ijheatmasstransfer.2016.06.078.

[237] P. Carrere, M. Prat, Liquid water in cathode gas diffusion layers of PEM fuel cells: Identification of yarious pore filling regimes from pore network simulations, Int. J. Heat Mass Transf. 129 (2019) 1043-1056. doi:10.1016/j.ijheatmasstransfer.2018.10.004.

[238] M. El Hannach, J. Pauchet, M. Prat, Pore network modeling: Application to multiphase transport inside the cathode catalyst layer of proton exchange membrane fuel cell, Electrochim. Acta. 56 (2011) 10796-10808. doi:10.1016/j.electacta.2011.05.060. 
[239] P.P. Mukherjee, Q. Kang, C.Y. Wang, Pore-scale modeling of two-phase transport in polymer electrolyte fuel cells - Progress and perspective, Energy Environ. Sci. 4 (2011) 346369. doi:10.1039/b926077c.

[240] M. El Hannach, M. Prat, J. Pauchet, Pore network model of the cathode catalyst layer of proton exchange membrane fuel cells: Analysis of water management and electrical performance, Int. J. Hydrogen Energy. 37 (2012) 18996-19006. doi:10.1016/j.ijhydene.2012.09.139.

[241] R. Wu, Q. Liao, X. Zhu, H. Wang, Pore network modeling of cathode catalyst layer of proton exchange membrane fuel cell, Int. J. Hydrogen Energy. 37 (2012) 11255-11267. doi:10.1016/j.ijhydene.2012.04.036.

[242] L. Hao, P. Cheng, Lattice Boltzmann simulations of anisotropic permeabilities in carbon paper gas diffusion layers, J. Power Sources. 186 (2009) 104-114. doi:10.1016/j.jpowsour.2008.09.086.

[243] J. Park, X. Li, Application of Lattice Boltzmann Method to the Simulation of Multiphase Flow in the Inhomogeneous Porous Electrode of a PEM Fuel Cell, J. Power Sources. 173 (2008) 81-92. doi:10.1149/1.2921535.

[244] Y. Gao, X. Zhang, P. Rama, R. Chen, H. Ostadi, K. Jiang, Lattice Boltzmann simulation of water and gas flow in porous gas diffusion layers in fuel cells reconstructed from microtomography, Comput. Math. with Appl. 65 (2013) 891-900. doi:10.1016/j.camwa.2012.08.006.

[245] L. Chen, H. Luan, Y. He, W. Tao, International Journal of Thermal Sciences Pore-scale fl ow and mass transport in gas diffusion layer of proton exchange membrane fuel cell with interdigitated fl ow fi elds, Int. J. Therm. Sci. 51 (2012) 132-144. doi:10.1016/j.ijthermalsci.2011.08.003.

[246] B. Han, H. Meng, Numerical studies of interfacial phenomena in liquid water transport in polymer electrolyte membrane fuel cells using the lattice Boltzmann method, Int. J. Hydrogen Energy. 38 (2013) 5053-5059. doi:10.1016/j.ijhydene.2013.02.055.

[247] G.R. Molaeimanesh, M.H. Akbari, Impact of PTFE distribution on the removal of liquid water from a PEMFC electrode by lattice Boltzmann method, Int. J. Hydrogen Energy. 39 (2014) 8401-8409. doi:10.1016/j.ijhydene.2014.03.089.

[248] G.R. Molaeimanesh, M.H. Akbari, Role of wettability and water droplet size during water removal from a PEMFC GDL by lattice Boltzmann method, Int. J. Hydrogen Energy. 41 (2016) 14872-14884. doi:10.1016/j.ijhydene.2016.06.252. 
[249] Z. Liu, H. Wu, Numerical modeling of liquid-gas two-phase flow and heat transfer in reconstructed porous media at pore scale, Int. J. Hydrogen Energy. 41 (2016) 12285-12292. doi:10.1016/j.ijhydene.2016.05.025.

[250] X.-D. Niu, T. Munekata, S.-A. Hyodo, K. Suga, An investigation of water-gas transport processes in the gas-diffusion-layer of a PEM fuel cell by a multiphase MRT LBM, J. Power Sources. 172 (2007) 542-552. doi:10.1016/j.jpowsour.2007.05.081.

[251] T. Koido, T. Furusawa, K. Moriyama, An approach to modeling two-phase transport in the gas diffusion layer of a proton exchange membrane fuel cell, J. Power Sources. 175 (2008) 127-136. doi:10.1016/j.jpowsour.2007.09.029.

[252] J. Park, X. Li, Multi-phase micro-scale flow simulation in the electrodes of a PEM fuel cell by lattice Boltzmann method, J. Power Sources. 178 (2008) $248-257$. doi:10.1016/j.jpowsour.2007.12.008.

[253] Y. Lee, Y. Tabe, M. Kozakai, T. Chikahisa, Numerical simulation of liquid water and gas flow in a channel and a simplified gas diffusion layer model of polymer electrolyte membrane fuel cells using the lattice Boltzmann method, J. Power Sources. 193 (2009) 2431. doi:10.1016/j.jpowsour.2009.01.068.

[254] C. Song, W.-Q. Tao, H. Luan, Y. Feng, Y'.-L. He, L. Chen, Coupling between finite volume method and lattice Boltzmann method and its application to fluid flow and mass transport in proton exchange membrane fuel cell, Int. J. Heat Mass Transf. 55 (2012) 3834-3848. doi:10.1016/j.ijheatmasstransfer.2012.02.020.

[255] L. Chen, Y. Feng, C. Song, L. Chen, Y. He, W. Tao, International Journal of Heat and Mass Transfer Multi-scale modeling of proton exchange membrane fuel cell by coupling finite volume method and lattice Boltzmann method, Int. J. Heat Mass Transf. 63 (2013) 268-283. doi:10.1016/j.ijheatmasstransfer.2013.03.048.

[256] Y. Gao, Z. Hou, X. Wu, P. Xu, The impact of sample size on transport properties of carbonpaper and carbon-cloth GDLs: Direct simulation using the lattice Boltzmann model, Int. J. Heat Mass Transf. 118 (2018) 1325-1339. doi:10.1016/j.ijheatmasstransfer.2017.11.003.

[257] D. Froning, J. Brinkmann, U. Reimer, W. Lehnert, D. Stolten, 3D analysis , modeling and simulation of transport processes in fibrous microstructures , using the Lattice Boltzmann method, Electrochim. Acta. 110 (2012) 2009. doi:org/10.1016/j.electacta.2013.04.071.

[258] G.R. Molaeimanesh, M. Nazemian, Investigation of GDL compression effects on the performance of a PEM fuel cell cathode by lattice Boltzmann method, J. Power Sources. 359 (2017) 494-506. doi:10.1016/j.jpowsour.2017.05.078. 
[259] L. Hao, P. Cheng, International Journal of Heat and Mass Transfer Pore-scale simulations on relative permeabilities of porous media by lattice Boltzmann method, Heat Mass Transf. 53 (2010) 8-13. doi:10.1016/j.ijheatmasstransfer.2009.12.066.

[260] L. Hao, P. Cheng, Capillary pressures in carbon paper gas diffusion layers having hydrophilic and hydrophobic pores, Int. J. Heat Mass Transf. 55 (2012) 133-139. doi:10.1016/j.ijheatmasstransfer.2011.08.049.

[261] P. Satjaritanun, J.W. Weidner, S. Hirano, Z. Lu, Y. Khunatorn, S. Ogawa, S.E. Litster, A.D, Shum, I. V. Zenyuk, S. Shimpalee, Micro-Scale Analysis of Liquid Water Breakthrough inside Gas Diffusion Layer for PEMFC Using X-ray Computed Tomography and Lattice Boltzmann Method, J. Electrochem. Soc. 164 (2017) E3359-E3371. doi:10.1149/2.0391711jes.

[262] P. Satjaritanun, S. Hirano, A.D. Shum, I. V. Zenyuk, A.Z. Weber, J.W. Weidner, S. Shimpalee, Fundamental Understanding of Water Movement in Gas Diffusion Layer under Different Arrangements Using Combination of Direct Modeling and Experimental Visualization, J. Electrochem. Soc. 165 (2018) F1115-F1126. doi:10.1149/2.0201814jes.

[263] L. Chen, G. Wu, E.F. Holby, P. Zelenay, W.Q. Tao, Q. Kang, Lattice boltzmann pore-scale investigation of coupled physical-electrochemical processes in C/PT and non-precious metal cathode catalyst layers in proton exchange membrane fuel cells, Electrochim. Acta. 158 (2015) 175-186. doi:10.1016/j.electacta.2015.01.121.

[264] Y. Gao, Using MRT lattice Boltzmann method to simulate gas flow in simplified catalyst layer for different inlet-outlet pressure ratio, Int. J. Heat Mass Transf. 88 (2015) 122-132. doi:10.1016/j.ijheatmasstransfer.2015.04.031.

[265] W. Zheng, S.H. Kim, The Effects of Catalyst Layer Microstructure and Water Saturation on the Effective Diffusivity in PEMFC, J. Electrochem. Soc. 165 (2018) F468-F478. doi:10.1149/2.0711807jes.

[266] G.R. Molaeimanesh, M.A. Bamdezh, M. Nazemian, Impact of catalyst layer morphology on the performance of PEM fuel cell cathode via lattice Boltzmann simulation, Int. J. Hydrogen Energy. 43 (2018) 20959-20975. doi:10.1016/j.ijhydene.2018.09.076.

[267] G.R. Molaeimanesh, M.H. Akbari, Agglomerate modeling of cathode catalyst layer of a PEM fuel cell by the lattice boltzmann method, Int. J. Hydrogen Energy. 40 (2015) 5169-5185. doi:10.1016/j.ijhydene.2015.02.097.

[268] S. Shin, A.R. Kim, S. Um, Computational prediction of nanoscale transport characteristics and catalyst utilization in fuel cell catalyst layers by the lattice Boltzmann method, Electrochim. Acta. 275 (2018) 87-99. doi:10.1016/j.electacta.2018.04.138. 
[269] A.A. Shah, H. Al-Fetlawi, F.C. Walsh, Dynamic modelling of hydrogen evolution effects in the all-vanadium redox flow battery, Electrochim. Acta. 55 (2010) 1125-1139. doi:10.1016/j.electacta.2009.10.022.

[270] Q. Xu, T.S. Zhao, P.K. Leung, Numerical investigations of flow field designs for vanadium redox flow batteries, Appl. Energy. 105 (2013) 47-56. doi:10.1016/j.apenergy.2012.12.041.

[271] W.W. Yang, Y.L. He, Y.S. Li, Performance Modeling of a Vanadium Redox Flow Battery during Discharging, Electrochim. Acta. 155 (2015) 279-287. doi:10.1016/j.electacta.2014.12.138.

[272] M.D.R. Kok, A. Khalifa, J.T. Gostick, Multiphysics Simulation of the Flow Battery Cathode: Cell Architecture and Electrode Optimization, J. Electrochem. Soc. 163 (2016) A1408A1419. doi:10.1149/2.1281607jes.

[273] M.D.R. Kok, R. Jervis, T.G. Tranter, M.A. Sadeghi, D.J.L. Brett, P.R. Shearing, J.T. Gostick, Mass transfer in fibrous media with varying anisotropy for flow battery electrodes: Direct numerical simulations with 3D X-ray computed tomography, Chem. Eng. Sci. 196 (2019) 104-115. doi:10.1016/j.ces.2018.10.049.

[274] R. Banerjee, N. Bevilacqua, L. Eifert, R. Zeis, Characterization of carbon felt electrodes for vanadium redox flow batteries - A pore nétwork modeling approach, J. Energy Storage. 21 (2019) 163-171. doi:10.1016/j.est.2018.11.014.

[275] M. Doyle, Modeling of Galvanostatic Charge and Discharge of the Lithium/Polymer/Insertion Cell, J. Electrochem. Soc. 140 (1993) 1526. doi:10.1149/1.2221597.

[276] T.F. Fuller, Simulation and Optimization of the Dual Lithium Ion Insertion Cell, J. Electrochem. Soc. 141 (1994) 1. doi:10.1149/1.2054684.

[277] M. Doyle, Comparison of Modeling Predictions with Experimental Data from Plastic Lithium Ion Cells, J. Electrochem. Soc. 143 (1996) 1890. doi:10.1149/1.1836921.

[278] Y. Xie, J. Li, C. Yuan, Multi-physics modeling of lithium ion battery capacity fading process with SEI growth by elementary reaction kinetics, J. Power Sources. 248 (2013) 172-179. doi:10.1016/j.jpowsour.2013.09.059.

[279] D. Miranda, C.M. Costa, A.M. Almeida, S. Lanceros-Méndez, Computer simulation of the influence of thermal conditions on the performance of conventional and unconventional lithium-ion battery geometries, Energy. 149 (2018) 262-278. doi:10.1016/j.energy.2018.02.026.

[280] R. Zhao, J. Liu, J. Gu, The effects of electrode thickness on the electrochemical and thermal characteristics of lithium ion battery, Appl. Energy. 139 (2015) 220-229. doi:10.1016/j.apenergy.2014.11.051. 
[281] M. Ender, An extended homogenized porous electrode model for lithium-ion cell electrodes, J. Power Sources. 282 (2015) 572-580. doi:10.1016/j.jpowsour.2015.02.098.

[282] C.-F. Chen, A. Verma, P.P. Mukherjee, Probing the role of electrode microstructure in the lithium-ion battery thermal behavior, J. Electrochem. Soc. 164 (2017) E3146-E3158. doi:10.1149/2.0161711jes.

[283] A. Vadakkepatt, B. Trembacki, S.R. Mathur, J.Y. Murthy, Bruggeman's exponents for effective thermal conductivity of lithium-ion battery electrodes, J. Electrochem. Soc. 163 (2016) A119-A130.

[284] L. Wu, V. De Andrade, X. Xiao, J. Zhang, Phase Field Modeling of Coupled Phase Separation and Diffusion Induced Stress in Lithium Iron Phosphate Particles Reconstructed from Synchrotron Nano X-ray Tomography, J. Electrochem. Energy Convers. Storage. (2019) 1-22.

[285] M.M. Forouzan, B.A. Mazzeo, D.R. Wheeler, Modeling the Effects of Electrode Microstructural Heterogeneities on Li-Ion Battery Performance and Lifetime, J. Electrochem. Soc. 165 (2018) A2127-A2144. doi:10.1149/2.1281809jes.

[286] B. Yan, C. Lim, Z. Song, L. Zhu, Analysis of Polarization in Realistic Li Ion Battery Electrode Microstructure Using Numerical Simulation, Electrochim. Acta. 185 (2015) 125141. doi:10.1016/j.electacta.2015.10.086.

[287] A.A. Franco, A. Rucci, D. Brandell, C. Frayret, M. Gaberscek, P. Jankowski, P. Johansson, Boosting Rechargeable Batteries R \& D by Multiscale Modeling : Myth or Reality?, Chem. Rev. 119 (2019) 4569-4627. doi:10.1021/acs.chemrev.8b00239.

[288] B.L. Trembacki, A.N. Mistry, D.R. Noble, M.E. Ferraro, P.P. Mukherjee, S.A. Roberts, Editors' Choice-Mesoscale Analysis of Conductive Binder Domain Morphology in Lithium-Ion Battery Electrodes, J. Electrochem. Soc. 165 (2018) E725-E736. doi:10.1149/2.0981813jes.

[289] L. Zielke, T. Hutzenlaub, D.R. Wheeler, I. Manke, T. Arlt, N. Paust, R. Zengerle, S. Thiele, A combination of X-ray tomography and carbon binder modeling: Reconstructing the three phases of LiCoO2Li-ion battery cathodes, Adv. Energy Mater. 4 (2014) 2-7. doi:10.1002/aenm.201301617.

[290] G.J. Nelson, L.J. Ausderau, S. Shin, J.R. Buckley, A. Mistry, P.P. Mukherjee, V. De Andrade, Transport-Geometry Interactions in Li-Ion Cathode Materials Imaged Using X-ray Nanotomography, J. Electrochem. Soc. 164 (2017) A1412-A1424. doi:10.1149/2.0261707jes. 
[291] M. Simon, J. Eller, V. Wood, M. Ebner, C. Burns, J. Dahn, Quantifying Inhomogeneity of Lithium Ion Battery Electrodes and Its Influence on Electrochemical Performance, 165 (2018) 339-344. doi:10.1149/2.0311802jes.

[292] B. Yan, C. Lim, L. Yin, L. Zhu, Simulation of heat generation in a reconstructed LiCoO2 cathode during galvanostatic discharge, Electrochim. Acta. 100 (2013) 171-179. doi:10.1016/j.electacta.2013.03.132.

[293] G.L. Sang, H.J. Dong, Effect of electrode compression on the wettability of lithium-ion batteries, J. Power Sources. 265 (2014) 363-369.

[294] Z. Jiang, Z. Qu, Lattice Boltzmann simulation of ion and electron transport in lithium ion battery porous electrode during discharge process, Energy Procedia. 88 (2016) 642-646. doi:10.1016/j.egypro.2016.06.091.

[295] S. He, B.T. Habte, F. Jiang, LBM prediction of effective electric and species transport properties of lithium-ion battery graphite anode, Solid State Ionics. 296 (2016) 146-153. doi:10.1016/j.ssi.2016.09.021.

[296] S.K. Mohammadian, Y. Zhang, Improving wettability and preventing Li-ion batteries from thermal runaway using microchannels International Journal of Heat and Mass Transfer Improving wettability and preventing $\mathrm{Li}$-ion batteries from thermal runaway using microchannels, Int. J. Heat Mass Transf. 118 (2017) 911-918. doi:10.1016/j.ijheatmasstransfer.2017.11.063.

[297] P. Barai, P.P. Mukherjee, Stochastic analysis of diffusion induced damage in lithium-ion battery electrodes, J. Electrochem. Soc. 160 (2013) A955-A967. doi:10.1149/2.132306jes.

[298] D.E. Galvez-Aranda, A. Verma, K. Hankins, J.M. Seminario, P.P. Mukherjee, P.B. Balbuena, Chemical and mechanical degradation and mitigation strategies for Si anodes, J. Power Sources. 419 (2019) 208-218. doi:10.1016/j.jpowsour.2019.02.054.

[299] N. Kotak, P. Barai, A. Verma, A. Mistry, P.P. Mukherjee, Electrochemistry-mechanics coupling in intercalation electrodes, J. Electrochem. Soc. 165 (2018) A1064-A1083. doi:10.1149/2.0621805jes.

[300] B. Wu, W. Lu, Journal of the Mechanics and Physics of Solids A consistently coupled multiscale mechanical - electrochemical battery model with particle interaction and its validation, J. Mech. Phys. Solids. 125 (2019) 89-111. doi:10.1016/j.jmps.2018.12.005.

[301] C. Sangrós, C. Schilde, L. Froböse, S. Ivanov, A. Kwade, Mechanical, electrical and ionic behavior of lithium - ion battery electrodes via DEM simulations, Energy Technol. (n.d.).

[302] A. Verma, T. Kotaka, Y. Tabuchi, P.P. Mukherjee, Mechano-Electrochemical Interaction and Degradation in Graphite Electrode with Surface Film, 165 (2018). doi:10.1149/2.1331810jes. 
[303] X. Duan, W. Jiang, Y. Zou, W. Lei, Z. Ma, A coupled electrochemical-thermal-mechanical model for spiral-wound Li-ion batteries, J. Mater. Sci. 53 (2018) 10987-11001. doi:10.1007/s 10853-018-2365-6.

[304] Y. Zhang, C. Zhao, Z. Guo, International Journal of Mechanical Sciences Simulation of crack behavior of secondary particles in Li-ion battery electrodes during lithiation/ delithiation cycles, Int. J. Mech. Sci. 155 (2019) 178-186. doi:10.1016/j.ijmecsci.2019.02.042.

[305] T. Hofmann, D. Westhoff, J. Feinauer, H. Andrä, J. Zausch, V. Schmidt, R. Müller, Electrochemo-mechanical simulation for lithium ion batteries across the scales, Int. J. Solids Struct. (2019). doi:10.1016/j.ijsolstr.2019.05.002.

[306] J. Zhu, T. Wierzbicki, W. Li, Review article A review of safety-focused mechanical modeling of commercial lithium-ion batteries, J. Power Sources. 378 (2018) 153-168. doi:10.1016/j.jpowsour.2017.12.034.

[307] Y. Zhao, P. Stein, Y. Bai, M. Al-Siraj, Y. Yang, B.X. Xu, A review on modeling of electrochemo-mechanics in lithium-ion batteries, J. Power Sources. 413 (2019) 259-283. doi:10.1016/j.jpowsour.2018.12.011.

[308] B. Wu, W. Lu, A battery model that fully couples mechanics and electrochemistry at both particle and electrode levels by incorporation of particle interaction, J. Power Sources. 360 (2017) 360-372. doi:10.1016/j.jpowsour.2017.05.115. 